А.У. Умбетов

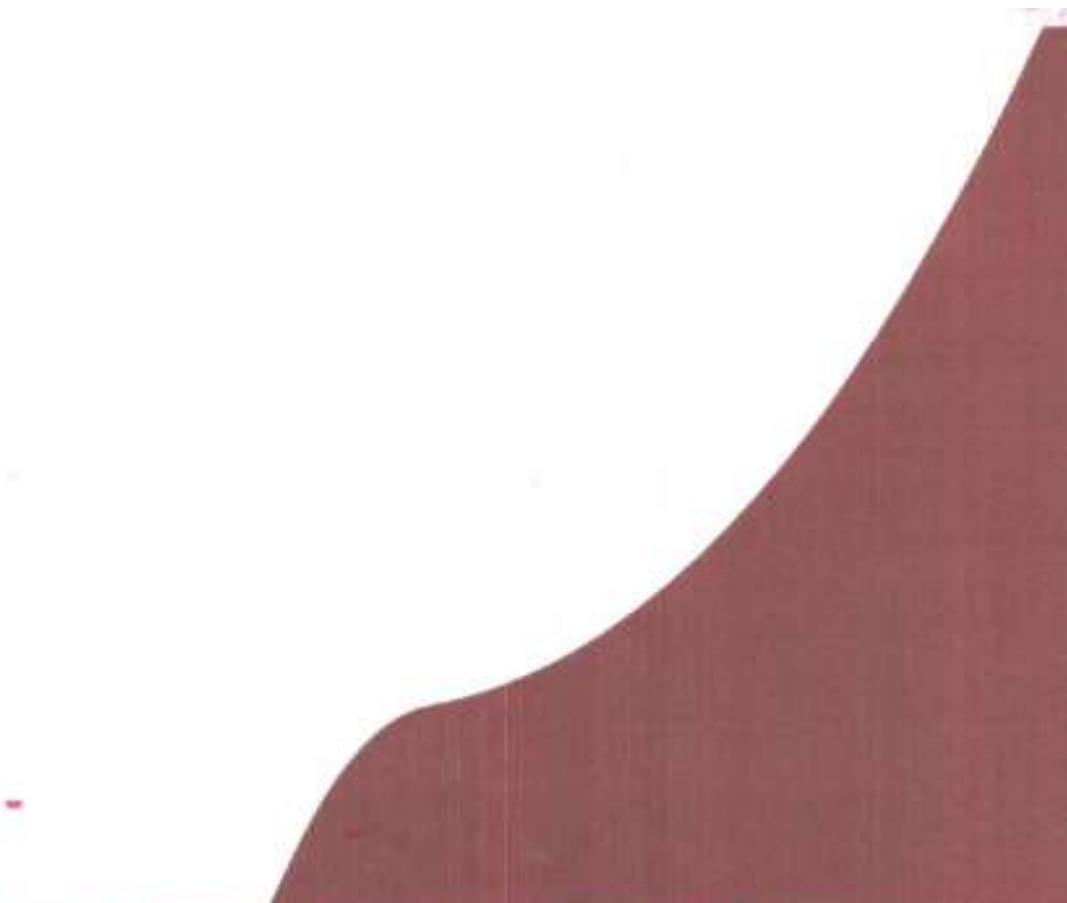

PAЗРАБОПКА И ИССЛЕДОВАНИЕ УСТРОЙСТВ ФОРМИРОВАКИЯ ОПТИЧЕСКИХ ИМПУЛЬСОВ

(Монография)

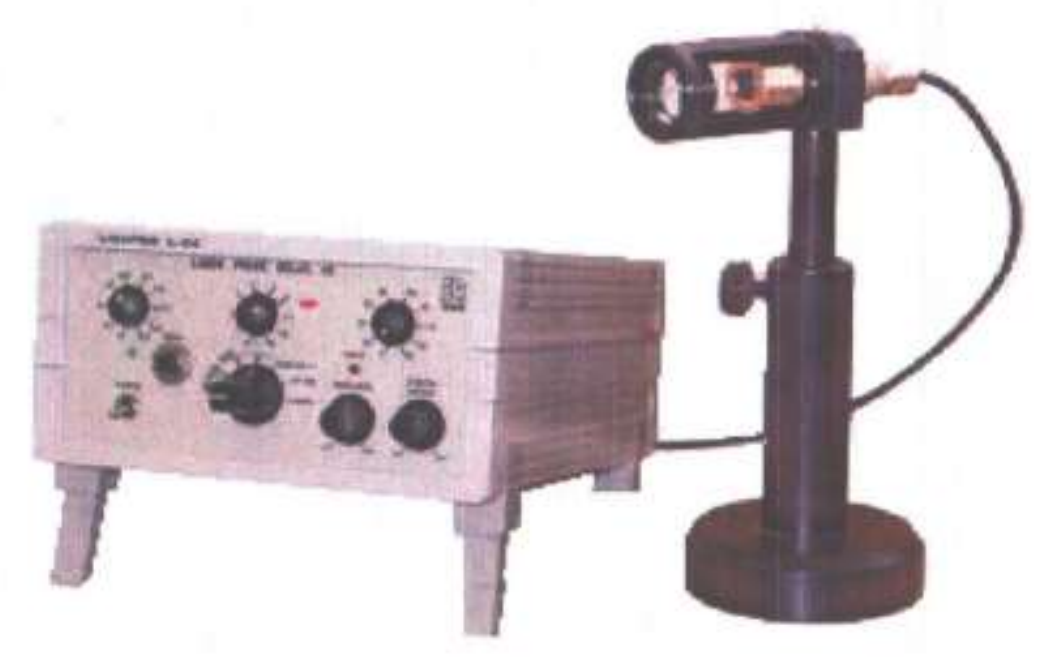


Министерство образования и науки Республики Казахстан Аркалыкский государственный педагогический институт имени И.Алтынсарина

\author{
А.У.Умбетов
}

\title{
Разработка и исследование устройств формирования оптических импульсов
}


УДК $530.1(075.8)$

ББК 22.336173

У 52

Рассмотрено на научно-методическом Совете Аркалыкского государственного педагогического института им. И.Алтынсарина и рекомендовано решением Ученого Совета. Протокол № 12.2014 г.

Рецензенты: Акылбеков А.Т. - декан физико-технического факультета ЕНУ им.Л.Н.Гумилева, доктор физика -математических наук, профессор

Кенжебеков Б.Т. - доктор педагогических наук, профессор ЕНУ им.Л.Н.Гумилева

\section{У 52 Умбетов А.У.}

Разработка и исследование устройств формирования оптических импульсов: монография.- Аркалык; АркГПИ, 2014.-96 с.

ISBN 978-601-7425-60-9

В работе рассмотрено создание импульсных источников света, включая лазерные источники перестраиваемой длительностью и частотой следования импульсов. Актуальность работы свзана с интенсивными ведущимися прикладными исследованиями в области оптической локации, связи и оптических методов обработки информации.

Рассмотрено также использование оптикоакустических пространственных модуляторов и ультразвуковых модуляторов света для ввода информации в оптическую систему, которые позволяют создать устройств обработки сигналов в реальном масштабе времени.

Работа представляет интерес для специалистов и студентов специальности «Физика», занимающихся вопросами разработки и исследования устройств формирования оптических импульсов.

ISBN 978-601-7425-60-9

УДК 530.1(075.8)

ББК 23.344Я73

(C) Умбетов А.У.

(C) АркГПИ им И.Алтынсарина, 2014 


\section{Содержание}

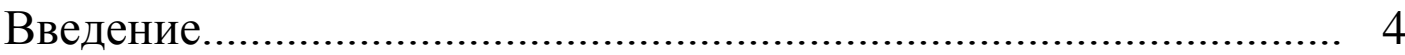

Глава I. Устройства формирования оптических импульсов .................. 6

1.1. Импульсный источник света микросекундной длительности ...... 6

1.2. Устройство формирования оптических импульсов 7

наносекундной длительности от лазера непрерывного

излучения.

$1.3 \quad$ Генерирование оптических импульсов полупроводниковыми 11

гетеролазерами

Глава II. Спектральный анализ оптических импульсов специальной формы .................................................................................................. 14

2.1. Энергетический спектр стационарного случайного процесса ...... 14

2.2. Энергетический спектр нестационарного случайного процесса .. 17

2.3. Энергетические спектры импульсных случайных

процессов................................................................... 20

2.4. Импульсные случайные процессы с независимыми

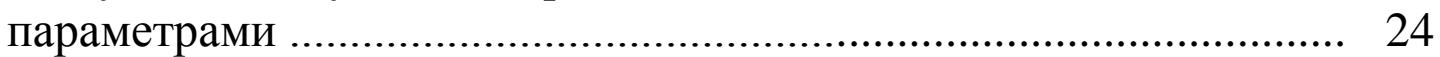

2.5. Импульсные случайные процессы с детерминированным тактовым интервалом........................................................... 25

2.6.

2.7. $\quad$ Спектры последовательностей импульсов..................................... 30

2.7.1. Последовательность экспоненциальных импульсов..................... 38

2.7.2. П Последовательность экспоненциальных импульсов, возникающих в случайные моменты времени............................. 39

Глава III. Особенности функционирования лазерных измерительных систем в каналах со случайными параметрами.......................... 40

3.1. $\quad$ Постановка задачи.................................................................. 40

3.2. Математическая модель комплекса пространственноразнесенных лис

3.3. Модели полей облачных неоднородностей и вероятностные характеристики сеанса измерений.............................................. 44

3.4. Анализ эффективности применения комплекса лис в условиях облачных полей ................................................................... 49

Глава IV. Характеристики режимов работы оптикоакустических

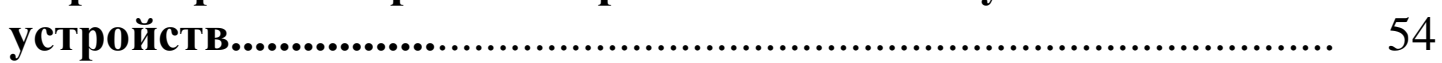

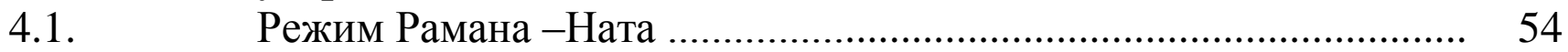

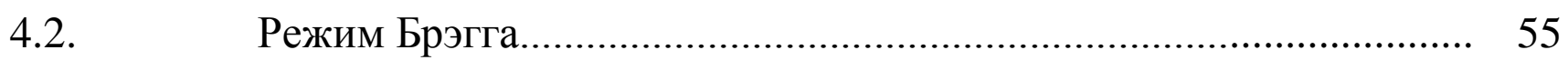

Глава V. Анализ оптикоакустических устройств обработки сигналов 61

5.1. Оптикоакустические гетеродинные устройства........................... 61

5.2. Магнитоакустооптический дефлектор............................................ 65

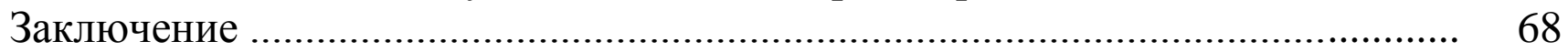

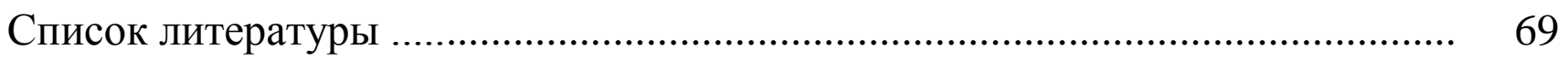

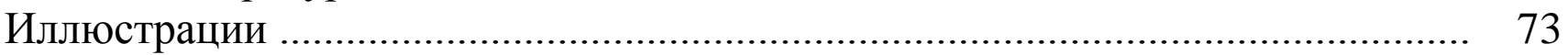




\section{Введение}

Известны различные устройства, генерирующие импульсы малой длительности: импульсные лазеры и импульсные лампы. Наряду с этим имеются устройства, вырабатывающие на выходе цветовые импульсы малой длительности при поступлении на вход непрерывного излучения. Это модуляторы различных типов: модулятор с ячейкой Керра, дифракционные модуляторы, электрооптический модулятор на эффекте Поккельса и др.Актуальность работ по созданию импульсных источников света, включая лазерные источники с перестраиваемой длительностью и частотой следования импульсов, связана с интенсивно ведущимися прикладными исследованиями в области оптической локации, связи и оптических методов обработки информации. Среди разработанных в настоящее время методов генерирования мощных коротких импульсов когерентного оптического излучения следует отметить метод использования в ОКГ резонаторов с управляемой добротностью с активными и пассивными оптическими затворами [I]. Таковы ОКГ на рубине и неодимовом стекле, для которых достигнуты длительности импульсов излучения порядка IO-20 нс. При этом скорость вращения зеркала является очень важным параметром ОКГ. Экспериментально установлено, что количество генерируемых импульсов при заданной мощности накачки уменьшается с увеличением скорости вращения зеркала, а мощность в импульсе заметно падает. Длительность серии импульсов обратно пропорциональна скорости вращения зеркала : $(\mathrm{IO}-60) \times \mathrm{IO}^{3}$ об/мин. Характерным параметром в кинетике образования коротких и мощных импульсов является время развития импульса генерации, которое зависит от размеров резонатора, уровня накачки, коэффициентов отражения в системе и для типичных условий составляет 0,2 мкс. В случае медленного включения оптического затвора излучение ОКГ имеет вид серии последовательных импульсов. При быстром включении затвора, происходящем за время, меньшее времени установления колебаний в генераторе, излучается вся запасенная в лазерном стержне энергия в виде одного импульса. В литературе [2-I5] описывается множества различных технических решений проблемы получения последовательности коротких оптических импульсов. Среди них заслуживают внимания устройства с превращением выходного излучения непрерывного лазера на красителе в серию импульсов длительностью 5 нс [2], неоновый лазер импульсного режима действия с генерацией оптических импульсов длительностью IOO нс при частоте следования импульсов I МГц [IO], лазер на алюмоиттриевом гранате с переключателем добротности для получения импульсов длительностью I,5- 2 мкс с частотой следования 4 кГц [II], инжекционные полупроводниковые лазеры в качестве быстродействующих излучателей для широкополосных оптических систем передачи информации на частотах модуляции порядка I -3 ГГц [I4].

Использование оптикоакустических (ОА) пронстранственных модуляторов, или ультразвукозых модуляторов света (УЗМС), для ввода 
информации в оптическую систему позволяет создать устройства обработки сигналов в реальном масштабе времени. В отечественной и зарубежной литературе принято включать в этот класс устройста (применительно к решению радиоэлектронных задач) устройства спектрального и корреляционного анализа сигналов и устройства голографической памяти сигналов.

Одной из главных областей применения ОА устройства является радиолокация.Основная операция, выполняемая ОА устройтвом при обработке принятого сигнала, которая позволила бы из слабого и притяженного импульса извлечь полезную информацию,- это операция свертки двух сигналов: исследуемого и опорного. Поэтому ОА устройство называют коррелятором ( или конвольвером). Объединяющим является часто термин « процессор». Достоинством ОА процессоров является реализуемость большой величины произведения Т $\Delta v,-\mathrm{T}$ - длительность импульса, $\Delta v-$ полоса частот. ОА процессоры могут работать либо в режиме Рамана-Ната, либо в режиме Брэгга. ОА процессоры, работающие в режиме Рамана-Ната ( несущая частота обычно ниже $100 \mathrm{MГц)} \mathrm{при} \mathrm{относительно} \mathrm{небольших}$ полосах пропускания могут иметь время обработки до нескольких сотен микросекунд, поскольку затухание звука на этих частотах обычно невелико. На более высоких частотах, где затухание звука возрастает, большие времена обработки получить трудно. ОА процессоры в режиме Брэгга обычно имеют времена обработки порядка нескольких десятков микросекунд, но при этом достижимы полосы частот до 100-300МГц. По способу выделения амплитудно модулированного сигнала ОА процессоры разделяются на когерентные и некогерентные. Когерентный метод выделения сигнала свертки иллюстрируется ниже на примере ОА процессора в режиме Рамана - Ната. Некогерентный метод детектирования характерен для ОА процессоров в режиме Брэгга. 


\section{Глава I \\ Устройства формирования оптических импульсов}

\section{1. Импульсный источник света микросекундной длительности}

Одним из возможных технических решений задачи формирования оптических импульсов с регулируемыми длительностью импульса и тактовой частотой является использование источников света с внутренней модуляцией. В созданном нами устройстве использована импульсная лампа типа ИСШ- IOO, режим работы которой задается специальным блоком питания. Данное устройства вырабатывает оптические импульсы с наибольшей энергией излучения в Уф области. Выбор данного источника связан с тем, что он дает возможность построить малогабаритное импульсное устройство многоцелевого назначения на широкую спектральную область от 240 нм до 800 нм. Кроме того Уф диапазон длин волн может представлять определенный интерес для оптических линий связи как в приземном слое атмосферы, так и в космическим пространстве. Это объясняется, во -первых, тем, что информационная емкость оптического приемопередатчика увеличивается с уменьшением длины волны излучения $\lambda$, и во -вторых, в при- еморегистрирующей аппаратуре с целью снижения уровня фоновой засветки при работе в дневное время от рассеянного солнечного излучения, максимум которого, как известно, лежит в видимой части спектра, возможно использование фотоприемника с максимумом чувствительности в Уф части спектра [I6,I7].

В настоящей работе исследованы импульсные лампы типа ИСШ, дающие УФ излучение большой интенсивности. Схема питания ламп на рис. I:I:У умформер УФ -I3-B, ПУ- поджигающее устройства, БП- блок питания поджигающего устройства. Тр -трансформатор 220/3500 В, КВкиловольтметр, $\mathrm{C}=0,05$ мкф (400 B), $\mathrm{R}=2,5$ кОм (75 Вт), АВC - I2-I- диоды. Лампы типа ИСШ представляют собой электрический газоразрядный источник кратковременных световых вспышек многократного действия. Баллон лампы изготавливается из увиолевого стекла с прозрачностью не менее 50\% для излучения с $\lambda=253,7$ нм (в расчете на толщину стенки баллона I мм). Габариты лампы: диаметр баллона 34 мм, высота лампы 85 мм, длина светящегося канала 3 мм. Баллон лампы наполняется ксеноном (или криптоном) при давлении 3 атм.Вспышка лампы происходит в момент пробоя газового промежутка между катодом и электродом зажигания, напряжению к которому подается от ПУ. Искра, образовавшаяся в результате пробоя промежутка, вызывает разряд конденсатора $\mathrm{C}$, сопровождающийся интенсивным световым излучением. На рис.1.2 представлены кривые спектрального распределения излучения ламп типа ИСШ: кривая “а” - для ИСШ- IOO -I, кривая “б”- для ИСШ - 500 при длительности вспышки 0,7 мкс (на уровне $\geq 35 \%$ от амплитудного значения силы света лампы). Кривые нормированы по главному максимуму излучения, расположенному в Уф части спектра и соответствующему области резонансной линии $\lambda=253,7$ нм. Амплитудное значение силы света составляет 
ориентировочно $2,5 \times 10^{4}$ кд, что соответствует световому потоку за время вспышки порядка $4,7 \times 10^{3}$ Вт. Интенсивный фон в видимой части спектра (рис.1.2) при необходимости можно убрать постановки на выходе соответствующего фильтра. Лампы ИСШ - IOO могут применяться в режиме одиночных световых импульсов при соответствующем выборе емкости С и частоты напряжения.

\section{2.Устройства формирования оптических импульсов наносекундной длительности от лазера непрерывного излучения}

Несмотря на то, что оптико - механические модуляторы светового потока не считаются современными, тем не менее с их помощью возможно решение поставленной задачи, а именно, получение световых импульсов наносекундной длительности. Такое техническое решение тем более желательно, что речь идет о создании лабораторного макета с использованием в нем доступного недорого оборудование на базе гелий - неонового лазера непрерывного действия и малогабаритного скоростного мотора на IO - 20 тысяч оборотов в минуту. Расчет оптико - механических модуляторов с вращающейся многогранной призмой рассматривается в работах [I8-I9] и применение сканирующего устройства на базе вращающихся многогранных зеркальных призм описывается в работе [20].

Проанализируем работу оптико - механического развертывающего устройства в виде вращающегося барабана - призмы с зеркальными гранями. Схематически рассмотрим ход лучей, представленный на рис. I.3, для общего случая немонохроматического источника света, обладающего диаграммой расходимости. $\Phi$ - фотоумножитель, $O_{I^{-}}$ось вращения призмы, $\mathrm{SS}^{\prime}$ - объектив, $0_{I} 0$ - оптическая ось объектива, $\mathrm{M}$ - источник света, $A_{I} B_{I}$ - положения зеркала в момент начала развертывания строки, $A_{2} B_{2}$-положение зеркала в момент начала развертывание строки, $B_{3} B_{I^{-}}$положение зеркала в момент начала развертывания следующей строки. Обозначения на рис. I.3 : L- расстояние от исследуемого источника до объектива, $\mathrm{K}$ - расстояние от объектива до оси вращения зеркального барабана, $\omega$ - угловая скорость вращения барабана $\left(\omega=2 \pi / t_{k}\right), \mathrm{R}$ - радиус зеркального барабана, $2 \psi-$ угол между радиусами, соединяющими крайние точки развертывающего зеркала и ось вращения $O_{I}(2$ $\psi=2 \frac{\pi}{m}$ где - число зеркал на барабане), d -диаметр отверстия ( диафрагмы) перед фотоприемником, $l$ - расстояние от объектива до диафрагмы до объектива, $\beta_{1} \beta_{2}$ - углы между осью $O_{I} \mathrm{O}$ и теми крайними лучами, в пределах которых при данных параметрах развертывающей системы должны лежать вся лучи, создающие изображение, для того, чтобы было полностью развернуто данным зеркалом, $\eta_{1}, \eta_{2^{-}}$углы между осью $O_{I} \mathrm{O}$ и направлениями на края развертывающего зеркала (линии $O_{I} B_{I}$ и $O_{I} A_{2}$ ) в моменты начала и конца развертывания данной строки, F- фокусное расстояние объектива. Для 
определения оптимальной расстановки элементов оптико-механического блока выводится формула, связывающая все выше- перечисленные параметры между собой, а также с периодом вращения зеркального барабана $t_{k}$, и линейной скоростью развертывания $\vartheta$ :

$$
v=\frac{4 \Psi m \ell t g\left[\frac{1}{2}\left(\beta_{1}-\beta_{2}\right)\right]}{\left[2 \psi-\left(\eta_{1}-\eta_{2}\right)\right] t_{k}}
$$

Кроме того, из рис.1,3 видно, что период развертывания одной строки, то - есть время перемещения зеркала из положения $\mathrm{A}_{1} \mathrm{~B}_{1}$ в положение $\mathrm{B}_{3} \mathrm{~B}_{1}$ равно: $t_{c}=t_{k} / m$. Это время разделяется на само время развертывания строки $t_{n}$ и на холостой ход, то - есть время перемещения зеркала из $\mathrm{A}_{2} \mathrm{~B}_{2}$ в $\mathrm{B}_{3} \mathrm{~B}_{1}$. После этого развертку своей строки начинает уже следующее зеркало. Максимальный угол сканирования $\Delta \beta_{\max }$, при котором обеспечивается полное отклонение светового пучка, зависит от числа граней $\mathrm{m}$ призмы, апертуры Д отклоняемого светового пучка, диаметра окружности $2 \mathrm{R}$, в которую вписывается многогранник сечения барабана - призмы. Этот угол может быть найден из выражения:

$$
\Delta \beta_{\max }=4\left[\arcsin \frac{D}{2 R \sin \left(\frac{\pi}{m}\right)}-\frac{\pi}{4}\right] ;
$$

Если максимальный угол отклонения задан и требуется определить радиус окружности барабана - призмы, то выражение (2) можно преобразовать к виду:

$$
R=\frac{D}{2 \sin \left(\frac{\pi}{4}+\frac{1}{4} \Delta \beta_{\max }\right) \sin \frac{\pi}{m}} ;
$$

Частота сканирования луча может быть найдена по соотношению: $v_{c}=m v_{g p}$, где $\quad v_{g p}$ - частота вращения призмы. Для увеличения частоты сканирования луча и полосы пропускания дефлекторов в виде зеркальных барабанов при заданной апертуре светового пучка обычно увеличивают число отражающих граней и скорость вращения. Увеличение числа отражающих граней влечет за собой увеличение габаритов дефлектора. Скорость вращения дефлектора можно повысить, используя в качестве привода, газовую турбину, приводимую во вращение сжатым воздухом, турбина может развивать скорость до $2 \times 10^{4} \mathrm{c}^{-1}$ [21] . Однако, предельная скорость для зеркального барабана размерами $6 \times 15 \times 28 \mathrm{~mm}^{3}$ не превышает $4 \times 10^{3} \mathrm{c}^{-1}$, а с Ø $300 \mathrm{Mм}-3 \times 10^{3} \mathrm{c}^{-1}$. Для увеличения скорости сканирования оптического луча могут быть использованы также различного рода оптические угловые ускорители $[22,23]$. 
Из всех известных в настоящее время модуляторов, позволяющих получить наносекундные длительности световых импульсов наиболее простым и легко осуществимым является модулятор, предложенный Гарбуни, Фоглом и Гансеном [24]. Нами этот модулятор был усовершенствован и использован для построения имитатора оптических импульсов и испытания приемоусилительных устройств и определения их инерционности.

В качестве источника света был использован газовый гелий - неоновый оптический квантовый генератор типа ЛГ-52, имеющий непрерывное когерентное излучение на длине волны 632,8км. Поток излучения со стороны плоского зеркала имел мощность порядка 10 мВт. Диаметр пучка на выходе был порядка I мми угол расходимости излучения составлял порядка 3 угл.минут. На оправу ОКГ крепилась насадка с зеркалом, дозволяющая направлять излучаемый поток под углом 90 к оси ОКГ. Излучение ОКГ направлялось на устройство, показанное на рис. 1.4. На ось мотора типа ДРВ 5, вращающегося со скоростью 2 х 104об/мин., былазакреплена призма с восемью отражающими гранями из ответа $\mathrm{K}$ - 8. Площадь каждой грани $4 \mathrm{~cm}^{2}$. На платформе на расстоянии 10 см от центра призмы устанавливались неподвижные зеркала, закрепленные в оправах, позволяющих их юстировать должным образом, причем последнее из них (8) по ходу луча с целью фокусирования пучка на входную щель, установленную перед приемником, было вогнутым. В описываемой установке каждая грань врашающейся с угловой скоростью юпризмы, отражая пучок света, заставляет его смещаться в плоскости, параллельной платформе с угловой скоростью $2 \omega$. Длительность светового импульса модулятора может быть вычислена по формуле [24] :

$$
\tau=\frac{D+d}{2 m\llcorner m}
$$

где 2 - диаметр светового пучка, проектируемого на входную щель ФЭУ, $\alpha-$ - ширина входной щели ФЭУ, $m$ - число граней вращающейся призмы, $\mathcal{L}_{m^{-}}$ расстояние от последней грани вращающегося зеркала до щели, $\omega$ - угловая скорость. Подставляя в (1.4) значения 3) $D=\mathrm{I} \mathrm{мм,} \alpha=0,1 \mathrm{MM}, \quad m=8, \omega=$ 2000 рад/с и $\mathcal{L} m=$ I м, получаем длительность светового импульса $\tau=33$ нс. В качестве фотоприемника использовался ФЭУ-30, с анадной нагрузки которого СНИМАЛСя импульсный сигнал и регистрировался осциллографом типа С1-31 или И2-7. При измерении длительности Импульса следует учитывать "затягивание" сигнала из-за подводящего монтажа в кожухе, в котором установлен ФЭУ. Если расходимость светового пучка от ОКГ характеризовать углом $\alpha$, то при отражении пучка плоскими зеркалами расходимость не меняется, а при прохождении конечного пути сечение пучка возрастает. Так как в описываемом модуляторе лазерный пучок последовательно отражается от нескольких зеркал, то его сечение возрастает пропорционально пройденному пути. Если же последнее зеркало модулятора заменить сферическим, то выходящий лазерный пучок на расстоянии $\mathcal{L} m+x$ от него может быть сфокусирован. Радиус кривизны этого зеркала $Z_{m}$ легко определяется из условия фокусировки пучка в плоскости щели приемной 
системы. При условии, что лазерный лучок параксиален и. гомоцентричен радиус кривизны вогнутого зеркала выражается следующим соотношением:

$$
Z_{m}=\frac{2(l m+x)\left[\ell_{0}-\ell_{1}+(2 m-3) x\right]}{L m+x+\ell_{0}+\ell_{1}+(2 m-3) x}
$$

где $\mathrm{X}$-расстояние от неподвижного зеркала до центра грани вращающейся призмы, $\ell_{0}=\phi / 2 \operatorname{tg} \frac{\alpha}{2} u \phi-$ диаметр выходного светового пятна лазера, $\ell_{1-}$ расстояние от лазера до первой отражающей грани вращающейся призмы. При фокусировке пучка лучний на входную щель, установленную перед ФЭУ, энергия, попадающая на ФЭУ, будет максимальной, обеспечивающей минимальную длительность светового импульса.

При фокусировке светового пучка от ОКГ величина амплитуды сигнала зависит от количества граней вращающейся призмы и от коэффициента отражения отражающих поверхностей, определяемых углами встречи луча с каждой из них по формулам, подобным формулам Френеля. Так как для металлических покрытий разница между коэффициентом отражения грани вращающейся призмы $\rho_{1}$ коэффициентом охранения неподвшшыхзеркал $p_{2}$ невелика,удобно ввести коэффициент $\quad p=\left(p_{1}+p\right) / 2$ характеризующий долю отраженного потока безотносительно к углу встречи падающего луча с отражающей поверхностью, тем более, что с увеличением количества зеркал разница между ними уменьшается.Тогда поток, попадающий на сфержеское зеркало и фокусируемый на щель приемной системы будет:

$$
\Phi=P^{(2 m-1)} B S \Omega \text {, }
$$

где $\mathrm{B}-$ яркость лазерного излучения, S- площадь светового пятна

$$
\Omega=4 \pi \sin ^{2} \frac{\alpha}{4}
$$

Полагая отношение сигнала к шуму $(\mathrm{C} / Ш)$ и $C=\gamma \phi, \quad$ где $\gamma$-чувствительность приемника, определим количество граней вращающейся призмы $\mathrm{m}$, обеспечивающих уверенный прием сигнала, из соотношения (1.2.4)

$$
m=\frac{1}{2}[(\lg \gamma \phi / \ell g \rho)-(\lg B S \Omega \gamma / \ell g p)+1]
$$

Выражения (1.2.7) и (1.2.4) позволяют определить минимальную длительность светового импульса модулятора, обеспечивающую заданную величину сигнала, вырабатываемого приемником. Действительно, подставляя (1.2.7) в (1.2.4), получаем:

$$
\tau=\frac{D+d}{\omega L_{m}\left[\left(\frac{\ell g \gamma \phi}{\ell g \rho}\right)-\left(\frac{\ell g B S \Omega Y}{\ell g \rho}\right)+1\right]}
$$


Таким образом, предлагаемое в настоящей работе устройство представляет собой надежным и удобный в работе генератор световых импульсов от гелий-неонового лазера, работающего в непрерывном режиме,использующий вращающуюся зеркальную призму.При увеличении числа граней вращающейся призмы до-восьми, использовании фокусирующего сферического зеркала и уменьшении диаметра лазерного пучка до 100 мкм длительность светового импульса может быть доведена до 4-5 нс. Зеркальный вариант модулятора делает универсальным его применение в УФ и ИК областях спектра. Модулятор может также найти применение для определения инерционности различных приемников оптико-электронных систем.

\section{3.Генерирование оптических,импульсов полупроводниковым гетеролазерами}

С точки зрения компактности и совершенства электронного управления длительностью и скважностью генерируемых оптических импульсов внимание привлекает использование инфекционных лазеров [25-33], в частности полупроводниковых гетеролазеров (ЯП) $[25,29,32]$. В работе [32] сообщалось об испытании волоконно-оптической линии передачи сигналов для систем дальней связи. При этом в качестве источника оптических импульсов были использованы ПГЛ полосксвой геометрии на системе $1 n-C a-A s-P$ с максимумом излучения в области 1,3 мкм. ПГЛ имеют низкие пороги накачки ( $\leq 0,5 \mathrm{~A}$ ), что позволяет их использование при ЗООК, а размер излучающей площадки $1 \times 16$ мкм (при угловой расходимости излучения 50 х 2 ) ) облегчает сопряжение микрооптических устройств в узле стыковки ПГЛ и стекловолоконногосветовода (СВC). В опытной линии передачи сигналов длиной 2 км сигнал регистрировался германиевым фотодиодом с темповым током $\sim 10^{-7} \mathrm{~A} \quad$ и чувствительностью $\sim 0,5 \frac{\mathrm{A}}{\mathrm{B} m}$ при $\lambda=1,3$ мкм. Малошумяшие широкополосные предварительное усилители и главный усилитель обеспечивали передачу информации со скоростью до 10 Мбит/с, минимальная обнаружимая мощность оптического сигнала на входе приемного устройства не превосходила $10^{-7}$ Вт.В экспериментальных установках желательно использование малощумящих фотодиодов или лавинных фотодиодов с усилением для регистрации оптических импульсов. В работе [зо] показано, что в излучении полупроводниковых лазеров (ПЛ) типа $C a A_{s}$ при $77 \mathrm{~K}$ на $\lambda=$ 902,8нм наблюдается аномальная динамака генерации излучения, характеризуемая тем, что с увеличением тока накачки оптический сигналне повторяет форму импульса накачки, а принимает Н - образную форму. Испытывались ПЛ длиной 350 мкм, шириной 180 мкм и толщиной активного слоя 1,7 мкм. Длительность импульса накачки составляла 250 нс,ток накачки менялся от $16 \mathrm{~A}$ до 29 А. Нами были проведены измерения временных характеристик ПГЛ в импульсном режиме. Испытывались ПГЛ созданные в ФТИ имени А.Ф.Иоффе на основе гетероструктур типа $1 n-G a,-A_{s}-P$ с полосковой геометрией. Ширина 
излучающей поверхности составляла 26 мкм. Предельные параметры работы ПГЛ: ток короткого замыкания (в импульсе) не более $0,6-0,75 \mathrm{~A}$, пороговое значение тока (7-II) $\frac{\mathrm{RA}}{c \mathrm{~A}}$. С помощью ПГЛ генерируются длинные $(\tau=0,5 \div 3 \mathrm{м \kappa c})$ и короткие (порядка десятков наносекунд) импульсы при частоте следования порядка $1 \div 2$ кГц. При комнатной температуре генерирование длинных импульсов приводит к разогреву ПГЛ, в результате чего срок службы ПГЛ существенно сокращается (до 50 - 100 часов). Возможность генерирования коротких импульсов, с другой стороны, ограничена временами жизни неосновных носителей порядка $4 \div 5$ нс. Структурная схема установки для измерения импульсных характеристик ПГЛ приведена на рис.1.5.ПГЛ крепился в медном держателе специальной конструкции между посеребренными «наковальней» и пружинным (плюсовым) контактом. Электрические импульсы прямоугольной формы от генератора (3) усиливались схемой формирования (2) импульсов тока накачки (рис.I.6) и подавались на ПГЛ (I).

Контроль уровня тока накачки осуществлялся измерителем импульсов (4). В случае формирования длинных ( $\tau \geq 0,5$ мкс) импульсов использовался генератор типа Г5-54 а в качестве измерителя - осциллограф C1-72, в случае коротких ( $\tau=20 \div 150$ ис) импульсов использовались генератор 15-48 и измеритель импульсов ИЧ-5. В качестве фотоприемника (ФП)использовались лавинный фотодиод марки ЛФД-2 для регистрации оптических импульсов от ПГЛ, работающих на длине волны $\lambda=0,9$ мкм, и германиевый фотодиод марки ЛФД-2 для регистрации импульсов излучения от ПГЛ на $\lambda=\mathrm{I}, 3$ мкм. Регистрирующем устройством служил осциллограф типа $\mathrm{C} 1-70$ с сменными стробоскопическим блоком типа Я40-2700 (IP71) и усилителем типа Я40-I700 (IУ7I). Схема включения ЛФД-2 приведена на рис.1.7. Результаты измерения временной формы оптических импульсов от некоторых ПГЛ приведены на осциллограммах (рис.1.8; $1.9 ; 1.10)$.

Одновременно приводятся осциллограммы импульсов накачки. На рис. 8 показана форма импульса излучения ПГЛ на $\lambda=1,3$ мкм. Из рис. 8 следует, что форма генерируемых оптических импульсов имеет «затяжку» переднего и заднего фронтов в случае длинных импульсов: $\tau \approx 2 \div 3$ мкс, (рис.1.8е,ж,3) с переходом на крутой (в пределе вертикальный) передний фронт для импульсов длительностью от 0,5 до 1,5 мкс. «Затяжка» заднего фронта связана с отсутствием согласования в электронных цепях между фотоприемником (ЛФД2) и осциллографом (СІ-72). Частота следования импульсов $(1 \div 10$ кГц $)$ задавалась генератором типа 15-54. На рис. 1.9 (а-ж) видно появление «звона» на заднем фронте для коротких импульсов излучения ПГЛ на $\lambda=0,9$ мкм с $\tau=15 \div 50 \mathrm{HC}$, что также характеризует отсутствие согласования в электронных цепях. Оптические импульсы на рис.1.9 получены от ПГЛ с $\lambda=0,9$ мкм при частоте следования импульсов 200 кГц. Регистрация импульсов производилась с помощью ФЭУ-62 и стребоскопического осциллографа типа C7-7. Импульс накачки формировался от генератора Г5-48. На рис. 1.10 приведена форма импульса накачки (при токе 155 мА, превышающем 
пороговое значение) длительностью 100 нс и импульса излучения ПГЛ на $\lambda=0,9$ мкм, зарегистрированного с помощью ЛФД-2 и осциллографа СI-70 через согласующий усилитель. При этом достигнута крутизна переднего фронта с длительностью нарастания 10 нс на уровне $(0,1 \div 0,9) U_{\max }$, а полуширина оптического импульса составляет 60 нс. Однако, задний фронт искажен («звон» остается неустраненным). Из этих примеров следует, что получение коротких ( $\tau \leq 100$ нс) оптических импульсов прямоугольный формы от ПГЛ затруднительно. Тем не менее существующие трудности не принципиальны, и их преодоление связано с решением схемотехнических вопросов. Работа в данном направлении продолжается.

С учетом реально получаемых форм оптических импульсов от ПГЛ, а именно: 1) с крутым передним и экспоненциальным задним фронтами, 2) с экспоненциальными передним и задним фронтами, - нами проведены расчеты спектров этих сигналов с целью возможного их использования в некоторых практических устройствах. 


\section{Глава 2 \\ Спектральный анализ оптических импульсов специальной формы}

\section{1 Энергетический спектр стационарного случайного процесса}

Случайный процесс $\xi(\mathrm{t})$ называется стационарным /строго/, если его функция распределения $\mathrm{W}_{\mathrm{n}}\left(\mathrm{x}_{1}, \mathrm{x}_{2}, \ldots, \mathrm{x}_{\mathrm{n}}, \mathrm{t}_{1}, \mathrm{t}_{2}, \ldots, \mathrm{t}_{\mathrm{n}}\right)$ произвольного порядка $n$ не меняется при любом сдвиге всей группы точек $\mathrm{t}_{1}, \mathrm{t}_{2}, \ldots, \mathrm{t}_{\mathrm{n}}$ вдоль оси времени, т.е. , если для любых $n$ и $\tau$ :

$$
\mathrm{W}_{\mathrm{n}}\left(\mathrm{x}_{1}, \mathrm{x}_{2}, \ldots, \mathrm{x}_{\mathrm{n}}, \mathrm{t}_{1}, \mathrm{t}_{2}, \ldots, \mathrm{t}_{\mathrm{n}}\right)=\mathrm{W}_{\mathrm{n}}\left(\mathrm{x}_{1}, \mathrm{x}_{2}, \ldots, \mathrm{x}_{\mathrm{n}}, \mathrm{t}_{1+} \tau, \mathrm{t}_{2+} \tau, \ldots, \mathrm{t}_{\mathrm{n}+} \tau\right) .
$$

Иначе говоря, случайный процесс будет стационарным, когда выраже функции распределения любого порядка не зависят от положения начала отсчета времени. Если вероятностные характеристики случайного процесса не инвариантны по отношению к произвольному смещению начала отсчета времени, то процесс нестационарный.

Из определения следует, что для стационарного случайного процесса: a/ одномерная функция распределения имеет один и тот же вид в любой момент времени/ т.е. не зависит от времени/:

$$
\mathrm{W}_{1}(\mathrm{x}, \mathrm{t})=\mathrm{W}_{1}(\mathrm{x}, \mathrm{t}+\tau)=\mathrm{W}_{1}(\mathrm{x})
$$

б/двумерная функция распределения может зависеть лишь от разности $\mathrm{t}_{2}-\mathrm{t}_{1}$ :

$$
\mathrm{W}_{2}\left(\mathrm{x}_{1}, \mathrm{x}_{2}, \mathrm{t}_{1}, \mathrm{t}_{2}\right)=\mathrm{W}_{2}\left(\mathrm{x}_{1}, \mathrm{x}_{2}, \mathrm{t}_{2}-\mathrm{t}_{1}\right),
$$

в/ трехмерная функция распределения может зависеть лишь от двух разностей $\mathrm{t}_{2}-\mathrm{t}_{1}, \mathrm{t}_{3}-\mathrm{t}_{1}$ :

$$
\mathrm{W}_{3}\left(\mathrm{x}_{1}, \mathrm{x}_{2}, \mathrm{x}_{3} \mathrm{t}_{1}, \mathrm{t}_{2}, \mathrm{t}_{3}\right)=\mathrm{W}_{3}\left(\mathrm{x}_{1}, \mathrm{x}_{2}, \mathrm{t}_{2}-\mathrm{t}_{1}, \mathrm{t}_{3}-\mathrm{t}_{1}\right) .
$$

Очевидно, что $n$-мерная функция распределения будет функцией только (n-1)го временного параметра $\mathrm{t}_{\mathrm{i}} \mathrm{t}_{1,} \mathrm{i}=2,3, \ldots \mathrm{n}$.

Поскольку двумерная функция распределения $\mathrm{W}_{2}\left(\mathrm{x}_{1}, \mathrm{x}_{2}, \mathrm{t}_{2}-\mathrm{t}_{1}\right)$ случайного стационарного процесса зависит только от разности $\tau=\mathrm{t}_{2^{-}} \mathrm{t}_{1}$, то и корреляционная функция такого процесса зависит только от одной переменной $\tau$ :

$$
\iint_{-\infty}^{\infty} x_{1} x_{2} W_{2}\left(x_{1} x_{2} \tau\right) d x_{1} d x_{2}
$$

Стационарность в широком смысле. Раздел теории, посвященный изучению лишь тех свойств случайных процессов, которые определяются их моментами первых двух порядков, называется корреляционной теорией. Поскольку в корреляционной теории многомерные распределения не фигурируют, то естественно считать в рамках этой теории стационарными все случайные процессы, у которых среднее значение и дисперсия не зависят от времени, а корреляционная функция зависит только от разности времен $\tau=\mathrm{t}_{2}$ $\mathrm{t}_{1}$. Случайные процессы, удовлетворяющие этим условиям называются стационарными в широком смысле /или стационарными в смысле А.Я.Хинчина/. 
Непосредственное приложение классического гармонического анализа к случайным процессам невозможно, т.к. для реализации этих процессов спектральные плотности по Фурье не имеют конечных значений при любых частотах.1) Можно обобщить, однако, гармонический анализ, усредняя спектральные разложения, полученные из отдельных реализаций.

Рассмотрим одну реализацию $\xi^{(\mathrm{k})}(\mathrm{t})$ случайного процесса $\xi(\mathrm{t})$. Пусть, кроме того, $\xi_{T}^{(x)}(\mathrm{t})$ - усеченная реализация, равная нулю вне интервала $/ \mathrm{t} / \leq \frac{T}{2}$ и совпадающая с $\xi^{(\mathrm{k})}(\mathrm{t})$ внутри этого интервала. Спектр /преобразование Фурье/ функции $\xi_{T}^{(x)}(\mathrm{t})$ имеет вид

$$
\mathrm{Z}_{T}^{(\mathrm{N})}(\omega)=\int_{-\frac{T}{2}}^{\frac{T}{2}} \xi_{T}^{(\mathrm{N})}(\mathrm{t}) e^{-i \omega t} d t
$$

Если $\xi_{T}^{(x)}(\mathrm{t})$ - напряжение или ток на нагрузке 1 Ом, то средняя мощность на частоте $\omega$, отнесенная к полосе $\Delta f=\frac{1}{T}$, равна

$$
G_{T}^{(x)}(\omega)=\frac{2}{T}\left|G_{T}^{(x)}(\omega)\right|^{2}=\frac{2}{T} \iint_{-\frac{T}{2}}^{\frac{T}{2}} \xi_{T}^{(x)}\left(\mathrm{t}_{1}\right) \xi_{T}^{(x)}\left(\mathrm{t}_{2}\right) e^{-i \omega\left(t_{1}-t_{2}\right)} d t_{1} d t_{2}
$$

При $T \rightarrow \infty$ случайная функция $G_{T}(\omega)$ не стремится, вообще говоря, к определенному пределу, а также является случайной

1) Преобразование Фурье справедливо только для функций, удовлетворяющих условию абсолютной интегрируемости $\int_{-\infty}^{\infty}|\xi(t)| d t<\infty$ Реализации случайного процесса $\xi(\mathrm{t})$ этому условию не удовлетворяют: для них интеграл расходится, т.к. подинтегральная функция при $t \rightarrow \infty$ предела не имеет велечиной. Среднее по множеству реализаций для $G_{t}(\omega)$ равно

$$
\begin{gathered}
F_{T}(\omega)=m_{1}\left\{G_{T} \omega\right\} \\
=\frac{2}{T} \iint_{-\frac{T}{2}}^{\frac{T}{2}} m_{1}\left\{_{T}\left(t_{1}\right)_{T}\left(t_{2}\right)\right\} e^{-i \omega\left(t_{1}-t_{2}\right)} d t_{1} d t_{2 .}
\end{gathered}
$$

Вводя корреляционную функцию $\mathrm{B}\left(t_{1} t_{2}\right.$ процесса $\xi(\mathrm{t})$, можно $F_{T}(\omega)$ представить в виде

$$
F_{T}=\frac{2}{T} \iint_{-T / 2}^{T / 2} B\left(t_{1}, t_{2}\right) e^{-i \omega\left(t_{1}-t_{2}\right)} d t_{1} d t_{2}
$$

Если случайный процесс $\xi(\mathrm{t})$ стационарный /по крайней мере в широком смысле/, то $B\left(t_{1}, t_{2}\right)=B\left(t_{1}-t_{2}\right)$ и, следовательно, 


$$
F_{T(\omega)}=\frac{2}{T} \iint_{-T / 2}^{T / 2} B\left(t_{1}-t_{2}\right) e^{-i \omega\left(t_{1}-t_{2}\right)} d t_{1} d t_{2}
$$

Разбивая область интегрирования $\left(t_{1}, t_{2}\right)$ в $(2.2)$ на две вдоль диагонали квадрата и вводя переменные $\tau=\left(t_{1}-t_{2}\right), t_{1}$ для области над диагональю $/ \tau>0 /$ и $\tau=\left(t_{1}-t_{2}\right),-t_{2}$ для области под диагональю $/ \tau>0 /$, полуаем (рис.2.1)

$$
\begin{array}{r}
F_{T(\omega)}=\frac{2}{T}\left[\int_{0}^{T} \int_{\tau-\frac{m}{2}}^{\frac{m}{2}} B(\tau) e^{-i \omega \tau} d t_{1} d \tau+\int_{0}^{T} \int_{-\frac{m}{2}}^{\tau+\frac{T}{2}} B(\tau) e^{-i \omega \tau} d t_{2} d \tau\right]= \\
=\frac{2}{T}\left[\int_{0}^{T}(T-\tau) B(\tau) e^{-i \omega \tau} d \tau+\int_{0}^{T}(T+\tau) B(\tau) e^{-i \omega \tau} d \tau\right]
\end{array}
$$

или

$$
F_{T(\omega)}=2 \int_{-T}^{T}\left(1-\frac{|\tau|}{T}\right) B(\tau) e^{-i \omega \tau} d \tau
$$

Предел $F_{T(\omega)}$ при $\mathrm{T} \rightarrow \infty$ равен

$$
F_{T(\omega)}=\lim _{T \rightarrow \infty} F_{T}(\omega)=\lim _{T \rightarrow \infty} m_{1}\left\{\frac{2}{T}\left|Z_{T}^{(K)}(i \omega)\right|^{2}\right\}=2 \int_{-\infty}^{\infty} B(\tau) e^{-i \omega \tau} d \tau,
$$

если интеграл в (2.1.3) существует, корреляционная функция $B(\tau)$ абсолютно интегрируема $/ \int_{-\infty}^{\infty}|B(\tau)| d \tau \leq M /$. Функцию частоты $\mathrm{F}(\omega)$, т.е.предел при $\mathrm{T} \rightarrow \infty$ усредненной по множеству реализаций спектральной плотности средней мощности процесса, называют энергетическим спектром стационарного /по крайней мере в широком смысле/ слуайного процесса. Этот спектр дает только усредненную картину распределения энергии по частотам элементарных гармонических составляющих, но не учитывает их фазовой структуры. Из (2.1.3) следует также, что энергетический спектр $\mathrm{F}(\omega)$ и корреляционная функция $B(\tau)$ стационарного случайного процесса связаны друг с другом парой преобразований Фурье /теорема Хинчина-Винера/

$$
\begin{gathered}
\mathrm{F}(\omega)=2 \int_{-\infty}^{\infty} B(\tau) e^{-i \omega \tau} d \tau=4 \int_{0}^{\infty} B(\tau) \cos \omega \tau d \tau, \\
B(\tau)=\frac{1}{4 \pi} \int_{-\infty}^{\infty} \mathrm{F}(\omega) e^{-i \omega \tau} d \omega=\frac{1}{2 \pi} \int_{0}^{\infty} \mathrm{F}(\omega) \cos \omega \tau \mathrm{d} \omega
\end{gathered}
$$

Так как преобразование фурье возможно только для абсолютно интегрируемых функций, то формулы (2.1.4) и (2.1.5) справедливы, если

$$
\int_{-\infty}^{\infty}|B(\tau)| d \tau \leq M \quad, \int_{-\infty}^{\infty}|F(\omega)| d \omega \leq N
$$

Эти условия органичивают применение теоремы Винера - Хинчина только для стационарных процессов, среднее значение которых равно нулю и у которых 
нет также квазидетерминированных составляющих. Если это условие выполнено, то энергетический спектр $F(\omega)$ стационарного случайного процесса - непрерывная функция частоты $\omega$.

Из (2.1.5) при $\tau=0$ находим, что средняя мощность стационарного процесса

$$
B(0)=\frac{1}{4 \pi} \int_{-\infty}^{\infty} F(\omega) d \omega
$$

равна площади его энергетического спектра. Спектральная плотность средней мощности при $\omega=0$

$$
F(0)=2 \int_{-\infty}^{\infty} B(\tau) d \tau
$$

равна удвоенной площади под кривой корреляционной функции.

\section{2. Энергетический спектр нестационарного случайного процесса}

Выделим снова одну реализацию $\xi^{(\mathrm{k})}(\mathrm{t})$ случайного процесса и сопоставим ей функцию $\xi_{\mathrm{T}}^{(\mathrm{k})}(\mathrm{t})$, равную нулю вне интервала $|\mathrm{t}| \leq \mathrm{T} / 2$ и совпадающую $\mathrm{c}$ $\xi^{(\mathrm{k})}(\mathrm{t})$ внутри этого интервала. Найдем текущий спектр усеченной реализации:

$$
S_{T}^{(k)}(t, \omega)=\int_{-T / 2}^{t} \mathfrak{I}_{\mathrm{T}}^{(\mathrm{k})}(t) e^{-i \omega t} d t
$$

Среднее по множеству реализаций мощности процесса $\xi_{\mathrm{T}}{ }^{(\mathrm{k})}(\mathrm{t})$ на частоте $\omega$ на интервале времени $\left(-\frac{T}{2}, \mathrm{t}\right)$ равно

$$
\begin{aligned}
& m_{1}\left\{\left.S_{T}^{(k)}(t, \omega)\right|^{2}\right\}=m_{1}\left\{\iint_{-\frac{T}{2}}^{t} \mathfrak{J}_{T}^{(k)}\left(t_{1}\right) \mathfrak{J}_{T}^{(k)}\left(t_{2}\right) e^{-i \omega\left(t_{1}-t_{2}\right)} d t_{1} d t_{2}\right\}= \\
& \iint_{-\frac{T}{2}}^{t} B_{T}\left(t_{1}, t_{2}\right) e^{-i \omega\left(t_{1}-t_{2}\right)} d t_{1} d t_{2},
\end{aligned}
$$

где

$$
B_{T}\left(t_{1}, t_{2}\right)=m_{1}\left\{\mathfrak{J}_{T}^{(k)}\left(t_{1}\right) \mathfrak{J}_{T}^{(k)}\left(t_{2}\right)\right\}
$$

Вводя в последнем интеграле переменную $\tau=\mathrm{t}_{1}-\mathrm{t}_{2}$ и разбивая область интегрирования на две, получаем 


$$
\begin{aligned}
& m_{1}\left\{\left.S_{T}^{(k)}(t, \omega)\right|^{2}\right\}=\int_{-\frac{T}{2}}^{t} \int_{0}^{t_{1}+T / 2} B_{T}\left(t_{1}, t_{1}-\tau\right) e^{-i \omega \tau} d \tau d t_{1}+\int_{-T / 2}^{t} \int_{\frac{T}{2}-t_{2}}^{0} B_{T}\left(t_{2}+\tau, t_{2}\right) e^{-i \omega \tau} d \tau d t_{2}= \\
& =\int_{-\frac{T}{2}}^{t} \int_{0}^{t_{1}+T / 2}\left[B_{T}\left(t_{1}, t_{1}-\tau\right) e^{-i \omega \tau}+B_{T}\left(t_{1}-\tau, t_{1}\right) e^{-i \omega \tau}\right] d \tau d t_{1},
\end{aligned}
$$$$
\text { и так как } \quad B_{T}\left(t_{1}, t_{2}\right)=B_{T}\left(t_{2}, t_{1}\right) \text { то, }
$$$$
m_{1}\left\{\left.S_{T}^{(k)}(t, \omega)\right|^{2}\right\}=2 \int_{-T / 2}^{t} \int_{0}^{t_{1}+T / 2} B_{T}\left(t_{1}, t_{1}-\tau\right) \cos \omega \tau d \tau d t_{1}
$$

Определим мгновенный энергетический спектр нестационарного случайного процесса согласно равенству

$$
\Phi(t, \omega)=\lim _{T \rightarrow \infty} \phi_{T}(t, \omega)
$$

где

$$
\Phi(t, \omega)=2 \frac{\partial}{\partial t} m_{1}\left\{S_{T}^{(k)}(t, \omega) \mid\right\}
$$

Дифференцируя (2.2.3) по t находим

$$
\Phi_{T}(t, \omega)=4 \int_{0}^{t+T / 2} B_{T}(t, t-\tau) \cos \omega \tau d \tau
$$

Переходя к пределу при $T \rightarrow \infty$,получаем

$$
\Phi(t, \omega)=4 \lim _{T \rightarrow \infty} \int_{0}^{t+T / 2} B_{T}(t, t-\tau) \cos \omega \tau d \tau=4 \int_{0}^{\infty} B(t, t-\tau) \cos \omega \tau d \tau
$$

и

$$
B(t, t-\tau)=\frac{1}{2 \pi} \int_{0}^{\infty} \phi(t, \omega) \cos \omega \tau d \tau,
$$

где $B\left(t_{1}, t_{2}\right)$ - корреляционная функция нестационарного случайного процесса $\xi(\mathrm{t})$.

Следовательно, мгновенный энергетический спектр и корреляционная функция нестационарного случайного процесса являются парой преобразований фурье по переменным $\omega и \tau$. 
Введем среднее по времени на интервале $\left(-\frac{T}{2}, \frac{T}{2}\right)$ энергетического спектра нестационарного случайного процесса

$$
F_{T}(\omega)=\frac{1}{T} \int_{-\frac{T}{2}}^{\frac{T}{2}} \phi_{T}(t, \omega) d t
$$

Подставляя (2.2.2a) в (2.2.4), находим

$$
F_{T}(\omega)=\frac{2}{T}\left[m_{1}\left\{\left|S_{T}^{(k)}\left(\frac{T}{2}, \omega\right)\right|^{2}\right\}-m_{1}\left\{\left|S_{T}^{(k)}\left(-\frac{T}{2}, \omega\right)\right|^{2}\right\}\right]
$$

Так как сагласно $\quad(2.1 .6) \quad S_{T}^{(k)}\left(-\frac{T}{2}, \omega\right)=0 \quad, \quad S_{T}^{(k)}\left(-\frac{T}{2}, \omega\right)=Z_{T}^{(k)}(i \omega) \quad$, то

$$
F_{T}(\omega)=\frac{2}{T} m_{1}\left\{\left.Z_{T}^{(k)}(i \omega)\right|^{2}\right\}
$$

где $\frac{2}{T}\left|Z_{T}^{(k)}(i \omega)\right|^{2}$, как было указано в предыдущем параграфе, есть средняя мощность процесса на частоте $\omega$, отнесенная к полосе частот $\Delta f=\frac{1}{T}$.

В соответствии с общим определением энергетическим спектром нестационарного случайного процесса называется предел

$$
F(\omega)=\lim _{T \rightarrow \infty} F_{T}(\omega)
$$

Таким образом, для нестационарного случайного процесса

$$
F(\omega)=\lim _{T \rightarrow \infty} \frac{2}{T} m_{1}\left\{\left.Z_{T}^{(k)}(i \omega)\right|^{2}\right\}=\lim _{T \rightarrow \infty} \frac{1}{T} \int_{-\frac{T}{2}}^{\frac{T}{2}} \Phi_{E}(t, \omega) d t
$$

Из (2.2.3) и (2.2.4) следует также, что энергетический спектр нестационарного случайного процесса связан преобразованием Фурье с усредненной по времени корреляционной функцией этого процесса:

$$
F(\omega)=\lim _{T \rightarrow \infty} \frac{4}{T} \int_{-T / 2}^{T / 2} \int_{0}^{t+T / 2} B_{T}(t, t-\tau) \cos \omega \tau d t=4 \int_{0}^{\infty} B(\tau) \cos \omega \tau d \tau,
$$

где 


$$
B *(\tau)=\lim _{T \rightarrow \infty} \frac{1}{T} \int_{-T / 2}^{T / 2} B_{T}(t, t-\tau) d t
$$

\section{3. Энергетические спектры импульсных случайных процессов}

Последовательность импульсов, параметры которых являются случайными величинами, называется импульсным случайным процессом.

Импульсный случайный процесс определяется бесконечным множеством реализаций, каждая из которых представляет собой последовательность импульсов. Выделим одну/ напр., $K-ю /$ из этих последовательностей и рассмотрим $2 N+1$ импульсов, расположенных по обе стороны от нулевого импульса, связанного с началом отсчета времениенных по обе стороны от нулевого импульса, связанного с началом отсчета времени. Обозначим

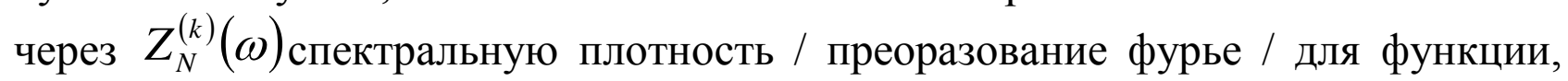
описывающей эту последовательность импульсов, и пусть расстояние между ее крайними импульсами равно $T_{N}=(2 N+1) T$, где $\mathrm{T}-$ среднее значение интервала между импульсами. Энергетический спектр импульсного процесса определяется из соотношения (2.2.5) :

$$
F(\omega)=\lim _{N \rightarrow \infty} \frac{2}{(2 N+1) T} m_{1}\left\{\left.Z_{N}^{(k)}(\omega)\right|^{2}\right\}
$$

Рассмотрим реализацию $\xi^{(\mathrm{k})}(\mathrm{t})$ импульного случайного процесса, у которого форма импульсов задана, а моменты их возникновения и окончания, а также амплитуды, случайны (рис.2.2)

Обозначим через $\varsigma_{n}^{(k)}\left(t-t_{n}^{(k)}, t_{n}^{(k)}+\tau_{n}^{(k)}\right)$ функцию, описывающую во времени $\mathrm{n}-$ й импульс К - й реализации. Эта функция должна тождественно обращаться в нуль вне интервала $\left(t_{n}^{(k)}, t_{n}^{(k)}+\tau_{n}^{k}\right)$. Пусть форма импульсов задается детерминированной функцией времени $\mathrm{U}(\mathrm{t})$, которая тождественно равна нулю вне интервала $0 \leq t \leq 1$.Импульсы любой реализации рассматриваемого случайного процесса получаются из $\mathrm{U}(\mathrm{t})$ умножением ее значений на величину $\xi^{(\mathrm{k})}(\mathrm{n})$, сдвигом по оси времени на величину $t_{n}^{(k)}$ и делением на величину $\tau_{n}^{k}$ - длительности $\mathrm{n}-г о$ импульса $\mathrm{K}$ - й реализации. Таким образом ,

$$
\varsigma_{n}^{(k)}\left(t-t_{n}^{(k)}, \tau_{n}^{(k)}\right)=\mathcal{J}_{n}^{(k)} U\left[\left(t-t_{n}^{(k)}\right) / \tau_{n}^{(k)}\right]
$$


Последовательность $2 N+1$ импульсов К - й реализации процесса может быть записана в виде

$$
\sum_{n=-N}^{N} \mathfrak{J}_{n}^{(k)} U\left[\left(t-t_{n}^{(k)} / \tau_{n}^{(k)}\right)\right]
$$

Обозначим через $\mathrm{g}(\omega)$ спектральную плотность / преобразование фурье / функции $\mathrm{U}(\mathrm{t})$ :

$$
g(\omega)=\int_{0}^{1} U(t) e^{-i \omega t} d t
$$

Тогда спектральная плотность для $\varsigma_{n}^{(k)}\left(t-t_{n}^{(k)}, \tau_{n}^{(k)}\right)$ равна

$$
\begin{gathered}
F_{n}^{(k)}\left(\omega ; t_{n}^{(k)}, \tau_{n}^{(k)}\right)=\mathfrak{J}_{n}^{(k)} \int_{t_{n}^{(k)}}^{t_{n}^{(k)}+\tau_{n}^{(k)}} U\left[\left(t-t_{n}^{(k)}\right) / \tau_{n}^{(k)}\right] e^{-i \omega t} d t= \\
=\mathfrak{J}_{n}^{(k)} \tau_{n}^{(k)} \int_{0}^{1} U(x) e^{-i \omega t\left(\tau_{n}^{(k)} x+t_{n}^{(k)}\right)} d x
\end{gathered}
$$

или

$$
F_{n}^{(k)}\left(\omega ; t_{n}^{(k)}, \tau_{n}^{(k)}\right)=\mathfrak{J}_{n}^{(k)} \tau_{n}^{(k)} g\left(\omega \tau_{n}^{(k)}\right) e^{-i \omega t_{n}^{(k)}}
$$

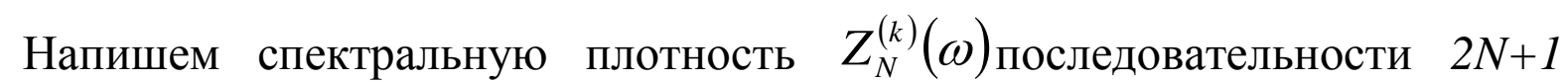
импульсов $K-\check{u}$ реализации процесса. Учитывая (2.3.2), находим

$$
Z_{n}^{(k)}(\omega)=\sum_{n=-N}^{N} \mathfrak{J}_{n}^{(k)} \tau_{n}^{(k)} g\left(\omega \tau_{n}^{(k)}\right) e^{-i \omega t_{n}^{(k)}}
$$

Для определения энергетического спектра импульсного случайного процесса подставим (2.3.3) в общую формулу (2.3.1):

$$
F(\omega)=\frac{2}{T} \lim _{N \rightarrow \infty} \frac{1}{2 N+1} m_{1}\left\{\left|\sum_{n=-N}^{N} \Im_{n}^{(k)} \tau_{n}^{(k)} g\left(\omega \tau_{n}^{(k)}\right) e^{-i \omega t_{n}^{(k)}}\right|^{2}\right\}
$$

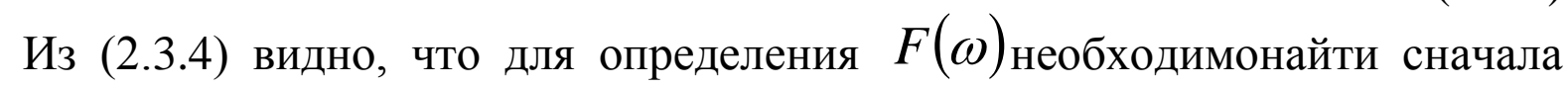
среднее по множеству / т.е. по индексуK/ от

Так как $\quad\left|Z_{n}^{(k)}(\omega)\right|=Z_{n}^{(k)}(\omega) \overline{Z_{n}^{(k)}(\omega)}$, то

$$
\frac{\left|Z_{n}^{(k)}(\omega)\right|^{2}}{\overline{Z_{n}^{(k)}(\omega)}, \quad \text { то }}
$$

$$
\left|Z_{n}^{(k)}(\omega)\right|^{2}=\sum_{n=-N}^{N} \sum_{j=-N}^{N} \mathfrak{J}_{n}^{(k)} \mathfrak{J}_{j}^{(k)} \tau_{n}^{(k)} \tau_{j}^{(k)} g\left(\omega \tau_{n}^{(k)}\right) \overline{g\left(\omega \tau_{j}^{(k)}\right)} e^{-i \omega t_{n}^{(k)}-t_{j}^{(k)}}
$$


Выделяя в двойной сумме члены, соответствующие $n=j и$, учитывая, что среднее суммы всегда равно сумме средних от слагаемых, находим

$$
\begin{aligned}
& m_{1}\left\{\left.Z_{N}^{(k)}(\omega)\right|^{2}\right\}=\sum_{n=-N}^{N} m_{1}\left\{\left[\mathfrak{J}_{n}^{(k)} \tau_{n}^{(k)}\right]^{2} \mid g\left(\omega \tau_{n}^{(k)}\right)^{2}\right\}+ \\
& +\sum_{n=-N}^{N} \sum_{j=-N}^{N} m_{1}\left\{\mathfrak{J}_{n}^{(k)} \mathfrak{J}_{j}^{(k)} \tau_{n}^{(k)} \tau_{j}^{(k)} g\left(\omega \tau_{n}^{(k)}\right) \overline{\left.g\left(\omega \tau_{j}^{(k)}\right) e^{-i \omega\left(t_{n}^{(k)}-t_{j}^{(k)}\right)}\right\} .}\right.
\end{aligned}
$$

Ограничимся только такими импульсными процессами, у которых статистические характеристики импульсов не зависят от его номера, а статистические характеристики совокупности импульсов зависят только от их взаимного расположения. При указанном ограничении величина

$$
K(\omega)=m_{1}\left\{\left[\xi_{n}^{(k)} \tau_{n}^{(k)}\right]^{2}\left|g\left(\omega \tau_{n}^{(k)}\right)\right|^{2}\right\}
$$

не зависит от номера импульса n, а величина

$$
\begin{aligned}
& n_{n-j}(\omega) \\
& =m_{1}\left\{\xi_{n}^{(k)} \xi_{j}^{(k)} \tau_{n}^{(k)} \tau_{j}^{(k)} g\left(\omega \tau_{n}^{(k)}\right) g\left(\omega \tau_{j}^{(k)}\right) e^{-i \omega\left(t_{n}^{(k)}-t_{j}^{(k)}\right)}\right\}
\end{aligned}
$$

Зависит только от разности $n-j$ номеров двух импульсов. Тогда

$$
\sum_{n=-N}^{N} m_{1}\left\{\left[\xi_{n}^{(k)} \tau_{n}^{(k)}\right]^{2}\left|g\left(\omega \tau_{n}^{(k)}\right)\right|^{2}\right\}=(2 N+1) K(\omega)
$$

а двойную сумму после некоторых преобразований можно представить в виде 


$$
\begin{aligned}
\sum_{n=-N}^{N} \sum_{j=-N}^{N} m_{1}\left\{\xi_{n}^{(k)} \xi_{j}^{(k)} \tau_{n}^{(k)} \tau_{j}^{(k)} g\left(\omega \tau_{n}^{(k)}\right) g\left(\omega \tau_{j}^{(k)}\right) e^{-i \omega\left(t_{n}^{(k)}-t_{j}^{(k)}\right)}\right\} \\
=\sum_{n=-N}^{N} \sum_{j=-N}^{N} n_{n-j}(\omega)=\sum_{p=1}^{2 N}(2 N+1-p)\left[n_{p}(\omega)+n_{p}(-\omega)\right], p \\
=n-j
\end{aligned}
$$

Подставляя (2.3.9) и (2.3.10) в (2.3.6) и учитывая (2.3.4), получаем общую формулу для энергетического спектра импульсного случайного процесса.

$$
\begin{aligned}
& F(\omega)=\frac{2}{T}\left\{\kappa(\omega)+\lim _{N \rightarrow \infty} \sum_{P=1}^{2 N}\left(1-\frac{P}{2 N+1}\right)\left[h_{p}(\omega)+h_{p}(-\omega)\right]\right\}=\frac{2}{T}[\kappa(\omega)+ \\
& \left.\lim _{N \rightarrow \infty} 2 \sum_{P=1}^{2 N}\left(1-\frac{P}{2 N+1}\right) \operatorname{Reh}_{p}(\omega)\right]
\end{aligned}
$$

Формула (2.3.11) дает наиболее общее выражение энергетического спектра импульсного случайного процесса с учетом взаимной корреляции его случайных параметров: амплитуд, длительностей и моментов возникновения импульсов.

Случайную последовательность импульсов неограниченной длительности аналитически можно записать в виде

$$
\xi_{(t)}^{(\mathrm{\kappa})}=\sum_{n=-\infty}^{\infty} e_{n}^{(k)}\left(t-t_{n}^{(k)}\right)=\sum_{n=-\infty}^{\infty} \xi_{n}^{(k)} U\left(t-t_{n}^{(k)}\right)
$$

где $e_{n}^{(k)}\left(t-t_{n}^{(k)}\right)$ - функция, описывающая К-ю реализацию $\mathrm{n}$-го импульса последовательности, $\xi_{n}^{(k)}$ - амплитуда - n-го импульса $K$-й реализации, $U\left(t-t_{n}^{(k)}\right)$-детерминированная функция времени, описывающая форму импульса. Выражение (2.3.1) справедливо и для случайной последовательности импульсов неограниченной длительности импульсов неограниченной длительности, если в формулах для $K(\omega)$ и $h_{p}(\omega)(2.3 .7)$ и (2.3.8) заменить на $\tau_{n}^{(k)} g\left(\omega \tau_{n}^{(k)}\right)$ на $g^{k}(\omega)$, вычесленную по формуле 


$$
g(\omega)=\int_{-\infty}^{\infty} u(x) e^{-i \omega x} d x
$$

Формулы (2.3.7) и (2.3.8)при этом преобразуется к виду:

$$
\begin{gathered}
K(w)=m_{1}\left\{\left[\xi_{n}^{(k)}\right]^{2}\right\}|g(\omega)|^{2} \\
h_{p}(\omega)=m_{1}\left\{\xi_{n}^{(k)} \xi_{j}^{(k)} e^{-i \omega\left[t_{n}^{(k)}-t_{j}^{(k)}\right]}\right\}|g(\omega)|^{2}, p=n-j
\end{gathered}
$$

\section{4 Импульсные случайные процессы с независимыми параметрами}

Предположим, что случайные параметры импульсного случайного процесса взаимно независимы, но будем считать ,что между однородными параметрами у различных импульсов существует корреляция. Введем вероятностные характеристики параметров импульсного случайного процесса. Обозначим через $\alpha, \delta^{2}$ среднее значение и дисперсию случайных амплитуд, $R_{p}$ коэффициент корреляции амплитуд $\mathrm{n}$-го и $\mathrm{j}-$ го импульсов $/ n-j=p$ $/, W_{1 \tau}(x), W_{2 \tau}\left(x W_{1 \tau}(x), y, p\right) \quad$-одномерную и двумерную распределения длительностей импульсов, $\tau_{0}, \quad \delta_{\tau}^{2}-$ средную длительность и дисперсию длительности импульса и $R_{p, \tau}$ - коэффицент корреляции длительностей $\mathrm{n}$-го и $\mathrm{j}$ - го импульса. Из (2.3.7) и (2.3.8) следует

$$
k(\omega)=\left(a^{2}+\delta^{2}\right) k_{0}(\omega)
$$

где

$$
\begin{gathered}
k_{0}(\omega)=\int_{0}^{\infty} x^{2}|g(\omega x)|^{2} W_{1 \tau}(x) d x \\
h_{p}(\omega)=\left(\delta^{2} R_{p}+a^{2}\right) K_{p}(\omega) H_{p}(\omega) \\
K_{p}(\omega)=\iint_{0}^{\infty} x y g(\omega x) g(\omega y) W_{2 \tau}(x, y, p) d x d y, \\
H_{p}(\omega)=m_{1}\left\{e^{-i \omega\left(t_{n}^{(k)}-t_{j}^{(k)}\right)}\right\}, \quad p=n-j
\end{gathered}
$$

Из (2.3.11) находим теперь следующее общее выражение энергетического спектра импульсного случайного процесса: 
$F(w)=$

$\frac{2}{T}\left\{\left(a^{2}+\delta^{2}\right) K_{0}(\omega)+\lim _{N \rightarrow \infty} 2 \sum_{P=1}^{2 N}\left(1-\frac{P}{2 N+1}\right)\left(\delta^{2} R_{p}+a^{2}\right) R_{e}\left[h_{p}(\omega) h_{p}(\omega)\right]\right\}$

В формуле (2.4.6) функция зависит только от совместного распределения моментов появления импульсов, а функция $K_{p(\omega)}$ зависит только от двумерного распределения длительностей импульсов.

Когда последовательность состоит из импульсов, у которых амплитуда и моменты возникновения случайны, а длительность неограниченны, формула (2.4.6) счетом (2.3.12) и (2.3.13) несколько упрощается:

$F(w)=\frac{2}{T}|g(\omega)|^{2}\left[a^{2}+\delta^{2}+\lim _{N \rightarrow \infty} 2 \sum_{P=1}^{2 N}\left(1-\frac{P}{2 N+1}\right)\left(\delta^{2} R_{p}+a^{2}\right) R_{e} H_{p}(\omega)\right]$

Случайные последовательности импульсов подразделяют на два класса в зависимости от детерминированности или случайности тактового интервала. К последовательностям с детерминированным тактовым интервалом относят как последовательности, для которых период следования импульсов равен постоянной величине $\mathrm{T}$, так и последовательности импульсов с периодом следования, случайно изменяющимся в определенных пределах относительно средного периода Т. Для последовательности с детерминированным тактовым интервалом характеризуется конечной, соизмеримой со средним периодом Т, дисперсией. C другой стороны, последовательности импульсов со случайным периодом следования, изменяющимся в принципе неограниченных пределах от нуля или от длительности импульса до значений, значительно превыщающих средний период Т, бесконечности, относятся к апериодическим импульсным случайным процессам.

\section{5 Импульсные случайные прцессы с детерминированным тактовым интервалом}

В импульсных случайных процессах с детерминированным тактовым интервалом момент появления $t_{n}^{(k)}$ любого $\mathrm{n}$-го импульса реализации процесса может быть представлен в виде

$$
t_{n}^{(k)}=n T+v_{n}^{(k)},
$$

где $\mathrm{T}$-длина тактового интервала; $v_{n}$-случайная величина с нулевым средним. Так как расмотриваемом случае за один такт возникает только один импульс, то $v_{n}$ не преосходит по абсолютному значению $\mathrm{T} / 2$.Пусть известны характеристические функции [34] $\theta_{1 v}(\omega) u\left(\omega_{1}, \omega_{2}, p T\right)$ случайны величины . Для расмотриваемой группы случайных процессов функция

$H_{p(\omega)}(2.4 .5)$ 
имеет вид

$$
\begin{gathered}
H_{p}(\omega)=e^{-i \omega p T} m_{1}\left\{e^{-i \omega\left(v_{n}^{(k)}-v_{j}^{(k)}\right)}\right\}, \quad p=n-j \quad \text { или } \\
H_{p}(\omega)=\theta_{2 v}(\omega,-\omega, p T) e^{-i \omega p T},
\end{gathered}
$$

В том случае,когда однордные параметры различных импульсов взаимно независимы.

$$
\begin{gathered}
R_{p}=0 \quad H_{p}(\omega)=\left|\theta_{1 v}(\omega)\right|^{2} e^{-i \omega p T} \\
K_{p}(\omega)=\left|\int_{0}^{\infty} x g(\omega x) W_{1 \tau}(x) d x\right|^{2}=K_{\infty}(\omega)
\end{gathered}
$$

Обозначим

$\Psi(\omega)=$

$\lim _{N \rightarrow \infty} 2 \sum_{P=1}^{2 N}\left(1-\frac{P}{2 N+1}\right)\left[K_{p}(\omega) \theta_{2 v}\left(\omega_{1}-\omega_{1} p T\right) e^{-i \omega p T}\right]+$

$\propto^{2} \operatorname{Re}\left(\left[K_{p}(\omega) \theta_{2 v}\left(\omega_{1}-\omega_{1} p T\right)-K_{\infty}(\omega) /\left|\theta_{1 v}(\omega)\right|^{2}\right] e^{-i \omega p T}\right.$

При взаимной независимости импульсов из (2.5.2) и (2.5.3) следует,что Используя (2.4.6) и (2.5.3),запишем общее выражение энергетического спектра импульсного случайного процесса с детерминированным тактовым интервалом:

$F(\omega)=\frac{2}{T}\left\{\left(a^{2}+\delta^{2}\right) K_{0}(\omega)-a^{2}\left|\theta_{1 v}(\omega)\right|^{2} K_{\infty}(\omega)+\psi(\omega)+a^{2}\left|\theta_{1 v}(\omega)\right|^{2} K_{\infty}(\omega) \lim _{N \rightarrow \infty}\left[1+2 \sum_{P=1}^{2 N}\left(1-\frac{P}{2 N+1}\right) \cos p \omega T\right]\right\}$

Можно показать [I],что

$$
\lim _{N \rightarrow \infty}\left[1+2 \sum_{P=1}^{2 N}\left(1-\frac{P}{2 N+1}\right) \cos p \omega T\right]=\frac{2 \pi}{T} \delta\left(\omega-\frac{2 \pi r}{T}\right),
$$

где $\delta(x)-\delta$-функция Дирака, $\mathrm{r}=0, \pm 1, \pm 2 \ldots$,

Подставляя (2.5.5) в (2.5.4), получаем общее выражение энергетического спектра импульсного случайного процесса с детерминированным тактовым интервалом.

$$
F(\omega)=\frac{2}{T}\left\{\left(a^{2}+\delta^{2}\right) K_{0}(\omega)-a^{2}\left|\theta_{1 v}(\omega)\right|^{2} K_{\infty}(\omega)+\psi(\omega)+a^{2}\left|\theta_{1 v}(\omega)\right|^{2} K_{\infty}(\omega) \frac{2 \pi}{T} \sum_{r=-\infty}^{\infty} \delta\left(\omega-\frac{2 \pi r}{T}\right)\right\}
$$

Если импульсы взаимно независимы, то в (2.5.6) следует положить $\psi(\omega)=0$

Общее выражение(2.5.6) энергетического спектра импульсного случайного процесса состоит из непрерывной части

$$
F_{h}(\omega)=\frac{2}{T}\left\{\left(a^{2}+\delta^{2}\right) K_{0}(\omega)-a^{2}\left|\theta_{1 v}(\omega)\right|^{2} K_{\infty}(\omega)+\psi(\omega)\right\}
$$


и дискретной части

$$
F_{g}(\omega)=\frac{4 \pi a^{2}}{T^{2}}\left|\theta_{1 v}(\omega)\right|^{2} K_{\infty}(\omega) \sum_{r=-\infty}^{\infty} \delta\left(\omega-\frac{2 \pi r}{T}\right)
$$

состоящей из дискретных линий на частотах, кратных частоте $\frac{2 \pi}{T}$ повторение импульсов. Заметим, что от корреляции импульсов зависи только непрерывная часть спектра.

Нетрудно видеть, что если параметры импульсов постоянны, то

$$
\theta_{1 \gamma}(\omega) \equiv 1, \psi(\omega) \equiv 0, K_{0}(\omega)=\left|\tau_{0} g\left(\omega \tau_{0}\right)\right|^{2}
$$

При этом непрерывная часть спектра исчезает, а дискретная часть совпадает со спектром мощности периодической последовательности немодулированных импульсов.

Отношения полной мощности составляющих непрерывного спектра к полной мощности дискретного спектра равно.

$$
\mu=\frac{\left.\int_{-\infty}{ }^{\infty}\left|\left[\alpha^{2}+\sigma^{2}\right) K_{0}(\omega)-\alpha^{2}\right| \theta_{1 \gamma}(\omega)\right|^{2} K_{\infty}(\omega)+\psi(\omega) \mid d \omega}{\frac{2 \pi a^{2}}{T} \sum_{r=-\infty}^{\infty}\left|\theta_{1 \gamma}\left(\frac{2 \pi r}{T}\right)\right|^{2} K_{\infty}\left(\frac{2 \pi r}{T}\right)}
$$

Поэтому в тех случаях, когда искажения импульсов вызваны помехами, можно отождествить непрерывную часть спектра (2.5.7) со спектром помехи, а дискретную (2.5.8) - со спектром сигнала. В этом случае отношения $\mu$, вычисленное по (2.5.9), даст отношения мощности помехи к мощности сигнала.

\section{6. Апериодические импульсные случайные процессы}

Рассмотрим импульсные случайные процессы, у которых нет детерминированного тактового интервала. Предположим, что интервалы между последовательными импульсами взаимно независимы. Обозначим интервал между моментами появления между моментами появления двух последовательных импульсов реализации через

$$
\mu_{n}^{(k)}=t_{n+1}^{(k)}-t_{n}^{(k)}
$$

и зададим характеристическую функцию $\theta_{1 \mu}(\omega)$ случайной величины $\mu_{n}$, предполагая, что это функция не зависит от n, т.е. от того, где расположен интервал $\mu_{n}$ на оси времени. Можно показать, что при указанном предположении импульсный процесс будет стационарным (в широком смысле).

Для исследования энергетического спектра рассматриваемого импульсного случайного процесса обратимся вновь к формуле (2.3.11).Если сохранить предположения о том, что отсутствует статистическая зависимость амплитуд импульсов от их длительности, то функция $\mathrm{K}(\omega)$ определяется по 
формулам (2.4.1) и(2.4.2). Для определения $h_{p}(\omega)$ нельзя воспользоваться (2.4.3), так как она получена в предположении. Что интервалы времени между моментами появления импульсов не зависят от их длительностей теперь необходимо отказаться от этого предположения. Действительно, интервал времени $\mu_{n}$ между моментами возникновения двух последовательных импульсов является суммой двух случайных величин: длительности импульса $\tau_{n}$ и длительности паузы между импульсам $\tau_{n}^{*}$. Следовательно, в общем случае случайные величины $\mu_{n}$ и $\tau_{n}$ зависимы. Обозначим через $\theta_{1 \tau}(\omega)$ и

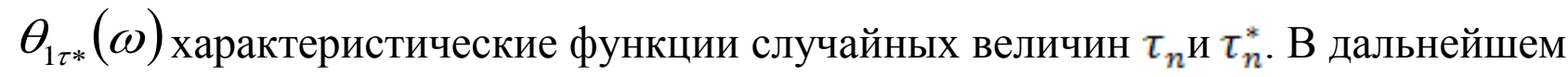
ограничимся случаем, когда длительности импульса и паузы независимы. Тогда

$$
\theta_{1 \mu}(\omega)=\theta_{1 \tau}(\omega) \theta_{1 \tau *}(\omega)
$$

Так как при $n \neq j$

$$
t_{n}^{(k)}-t_{j}^{(k)}=\tau_{j}^{(k)}+\tau_{j}^{*(k)}+\sum_{r=j+1}^{n-1} \mu_{r}^{(k)}
$$

то из (2.20) для некоррелированных амплитуд непосредственно следует

$$
\left.h_{n-j}(\omega)=a^{2} m_{1} \tau_{n}^{(k)} \tau_{j}^{(k)} g\left(\omega \tau_{n}^{(k)}\right) g\left(\omega \tau_{j}^{(k)}\right) \cdot e^{-i \omega \tau_{j}^{(k)}} e^{-i \omega \tau_{j}^{*(k)}} e p\left[-i \omega \sum_{r=j+1}^{n-1} \mu_{r}^{(k)}\right]\right\} \text {, }
$$

Где $\alpha$ - средные значение амплитуд.

В силу взаимной независимости случайных величин

$$
\tau_{j}, \tau_{j}^{*}, \tau_{n}, \tau_{n}^{*}, t_{n}+t_{j+1}=\sum_{r=j+1}^{n-1} \mu_{r}
$$

и независимости $\theta_{1 \mu}(\omega)$ от номера интервала имеем

$$
h_{n-j}(\omega)=a^{2} \theta_{1 \mu}^{n-j-1}(-\omega) \theta_{1 \tau^{*}}(-\omega) m_{1}\left\{\tau_{n}^{(k)} g\left(\omega \tau_{n}^{(k)}\right)\right\} m_{1}\left\{\tau_{j}^{(k)} g\left(\omega \tau_{j}^{(k)}\right) e^{-i \omega \tau_{j}^{(k)}}\right\}
$$

Учитывая, что

$$
g\left(\omega \tau_{j}^{(k)}\right) e^{-i \omega \tau_{j}^{(k)}}=\int_{0}^{1} U(t) e^{i \omega \tau_{j}^{(k)}(t-1)} d t=\int_{0}^{1} U(1-x) e^{-i \omega \tau_{j}^{(k)} x} d x
$$

и ввода обозначения:

$$
\begin{gathered}
g_{1}(\omega)=\int_{0}^{1} U(1-x) e^{-i \omega \tau_{j}^{(k)} x} d x=g(-\omega) e^{-i \omega}, \\
Q(\omega)=m_{1}\left\{\tau_{n}^{(k)} g\left(\omega \tau_{n}^{(k)}\right)\right\}=\int_{0}^{\infty} x g(\omega x) W_{1 \tau}(x) d x,
\end{gathered}
$$




$$
Q_{1}(\omega)=m_{1}\left\{\tau_{j}^{(k)} g_{1}\left(\omega \tau_{j}^{(k)}\right)\right\}=\int_{0}^{\infty} x g_{1}(\omega x) W_{1 \tau}(x) d x
$$

получаем

$$
h_{p}(\omega)=a^{2} Q(\omega) Q_{1}(\omega) \theta_{1 \tau *}(\omega) \theta_{1 \mu}^{p-1}(-\omega)
$$

Заметим, что

$$
\begin{aligned}
& Q(0)=Q_{1}(0)=\tau_{0} g(0), \quad \text { так как } \\
& \int_{0}^{1} U(x) d x=\int_{0}^{1} U(1-x) d x
\end{aligned}
$$

Подставляя (2.4.1) и (2.6.3) в (2.3.11) находим

$$
\begin{aligned}
& F(\omega)=\frac{2}{T}\left\{\left(a^{2}+\sigma^{2}\right) K_{0}(\omega)+\right. \\
& \left.+2 a^{2} \operatorname{Re}\left[Q(-\omega) Q_{1}(-\omega) \theta_{1 \tau *}(\omega)+\lim _{N \rightarrow \infty} \sum_{p=1}^{2 N}\left(1-\frac{P}{2 N+1}\right) \theta_{1 \mu}^{p-1}(\omega)\right]\right\}
\end{aligned}
$$

При $\omega \neq 0$

$$
\lim _{N \rightarrow \infty} \sum_{p=1}^{2 N}\left(1-\frac{P}{2 N+1}\right) \theta_{1 \mu}^{p-1}(\omega)=\frac{1}{1-\theta_{1 \mu}(\omega)}
$$

а при $\omega=0$ указанная сумма расходится и может быть представлена $\delta$ функцией.

Таким оброзом, имеем следующее аналитическое выражение энергетического спектра апериодического импульсного случайного процесса:

$$
\begin{aligned}
& F_{(\omega)}=\frac{2 a^{2}}{T}\left\{\left[1+\left(\frac{\sigma}{a}\right)^{2}\right] K_{0}(\omega)+\right. \\
& \left.+2 \operatorname{Re}\left[\frac{Q(-\omega) Q_{1}(-\omega) \theta_{1 \tau^{*}}(\omega)}{1-\theta_{1 \mu}(\omega)}\right]+\frac{g^{2}(0) \tau_{0}^{2}}{T} \delta(\omega)\right\}
\end{aligned}
$$

детерминированным тактовым интервалом (кроме одной дискретной компоненты, соответствующей постоянной составляющей процесса) энергетического спектра случайной последовательности импульсов неограниченной длительности, возникающих в случайные моменты времени, с некоррелированными амплитудами: 


$$
F_{(\omega)}=\frac{2 a^{2}}{T}|g(\omega)|^{2}\left\{1+\left(\frac{\sigma}{a}\right)^{2}+2 \operatorname{Re} \frac{\theta_{1 \mu}(\omega)}{1-\theta_{1 \mu}(\omega)}+\frac{1}{T} \delta(\omega)\right\}
$$

в котором $g(\omega)$ вычисляются по формуле $g(\omega)=\int_{-\infty}^{\infty} U(x) e^{-i \omega x} d x$.

\section{7. Спектры последовательностей импульсов}

Рассмотрим последовательность импульсов заданной формы, изображенной на рис. 2.3

Аналитически $n$ - и импульс $\mathrm{K}$ - и реализации такой последовательности

$$
\zeta_{n}^{(k)}\left(t-t_{n}^{(k)}, \tau_{n}^{(k)}\right)=\left\{\begin{array}{l}
\xi_{n}^{(k)}\left(1-e^{-\alpha\left(t-t_{n}^{(k)}\right)}\right), t_{n}^{(k)} \leq t \leq t_{n}^{(k)}+\tau_{n}^{(k)} \\
\xi_{n}^{(k)}\left(1-e^{-\alpha \tau_{n}^{(k)}}\right) e^{-\alpha\left(t-t_{n}^{(k)}-\tau_{n}^{(k)}\right)}, t>\mathrm{t}_{\mathrm{n}}^{(\mathrm{k})}+\tau_{n}^{(k)}
\end{array}\right.
$$

можно записать в виде

Спектральная плотность для $\varsigma_{n}^{(k)}\left(t-t_{n}^{(k)}, \tau_{n}^{(k)}\right)$ равна

$$
\begin{aligned}
& F\left(\omega, t_{n}^{(k)}, \tau_{n}^{(k)}\right)=\int_{t_{n}^{(k)}}^{\infty} \varsigma_{n}^{(k)}\left(t-t_{n}^{(k)}, \tau_{n}^{(k)}\right) e^{-i \omega t} d t= \\
& =\xi_{n}^{(k)} e^{-i \omega t_{n}^{(k)}}\left(\frac{1}{i \omega}-\frac{1}{\alpha+i \omega}\right)\left(1-e^{-i \omega \tau_{n}^{(k)}}\right)=\xi_{n}^{(k)} e^{-i \omega t_{n}^{(k)}} \chi(\omega, \alpha) g\left(\omega \tau_{n}^{(k)}\right),
\end{aligned}
$$

где введены обозначения

$$
\chi(\omega, \alpha)=\frac{1}{i \omega}-\frac{1}{\alpha+i \omega}, g\left(\omega \tau_{n}^{(k)}\right)=1-e^{-i \omega \tau_{n}^{(k)}}
$$

Для спектральной плотности $Z_{N}^{(k)}(\omega)$ последовательности $2 N+1$ импульсов К - и реализации процесса получаем

$$
Z_{N}^{(k)}(\omega)=\sum_{n=-N}^{N} \xi_{n}^{(k)} \chi(\omega, \alpha) g\left(\omega \tau_{n}^{(k)}\right) e^{-i \omega t_{n}^{(k)}}
$$


Далее, дословно повторяя выкладки п.3, находим выражение для энергетического спектра указанного импульсного случайного процесса:

$$
F(\omega)=\frac{2}{T}|\chi(\omega, \alpha)|^{2}\left\{K(\omega)+\lim _{N \rightarrow \infty} \sum_{p=1}^{2 N}\left(1-\frac{P}{2 N+1}\right) 2 \operatorname{Re} h_{p}(\omega)\right\}
$$

В формуле (2.7.5) выражения для $K(\omega)$ и $h_{p}(\omega)$ несколько отличаются от (2.3.7) и (2.3.8) и имеют вид:

$$
\begin{gathered}
K(\omega)=m_{1}\left\{\left(\xi_{n}^{(k)}\right)^{2} \mid g\left(\omega \tau_{n}^{(k)}\right)^{2}\right\} \\
h_{n-j}(\omega)=m_{1}\left\{\xi_{n}^{(k)} \xi_{j}^{(k)} g\left(\omega \tau_{n}^{(k)}\right) g\left(\omega \tau_{j}^{(k)}\right) e^{-i \omega\left(t_{n}^{(k)}-t_{j}^{(k)}\right)}\right\} n-j=p
\end{gathered}
$$

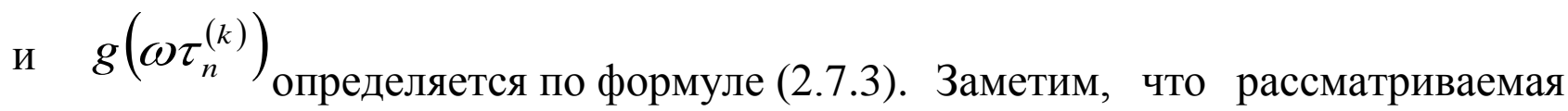
последовательность импульсов получается при прохождении последовательности прямоугольных импульсов через интегрирующую цепочку, передаточная функция которой равна $[35]$ :

$$
K=\frac{1}{1+\frac{\omega}{\alpha}} \quad \alpha=\frac{1}{R C}
$$

а квадрат частотной характеристики

$$
C^{2}(\omega)=\frac{1}{1+\frac{\omega^{2}}{\alpha^{2}}}
$$

В [34] показано, что энергетический спектр стационарного случайного процесса $F_{\xi}(\omega)$

при его прохождении через линейную систему с частотной характеристикой

$$
C(\omega)
$$

преобразуется по закону:

$$
F_{\varsigma}(\omega)=c^{2}(\omega) F_{\xi}(\omega)
$$

Следовательно, интересующий нас спектр последовательности импульсов (рис.2.3) можно получить, спользуя выражения для спектра последовательности прямоугольных импульсов, простым умножением на $C^{2}(\omega)$.

Мы будем исходит из формулы (2.7.5), дающей выражение для спектра последовательности импульсов (рис.2.3). Спектр последовательности прямоугольных импульсов следует из (2.7.5) при $\alpha \rightarrow \infty$. Если случайные параметры рассматриваемого нами импульсного случайного процесса взаимно независимы, то формула (2.7.8) принимает вид: 


$$
\begin{gathered}
F(w)=\frac{2}{T}|x(w, \alpha)|^{2}\left\{\left(\alpha^{2}+\delta^{2}\right) K^{0}(w)+\right. \\
\left.+\lim _{N \rightarrow \infty} 2 \sum_{P=1}^{2 N}\left(1-\frac{P}{2 N+1}\right)\left(\alpha^{2}+\delta^{2} R_{p}\right) R_{e}\left[K_{p}(w) H_{p}(w)\right]\right\},
\end{gathered}
$$

где

$$
K^{0}(w)=m_{1}\left\{\left|g\left(w \tau_{n}^{(K)}\right)\right|^{2}\right\}=\int_{0}^{\infty}|g(w x)|^{2} W_{1 \tau}(x) d x,
$$

$K_{p}(w)=m_{1}\left\{g\left(w \tau_{n}^{(K)}\right) g\left(w \tau_{\square}^{(K)}\right)\right\}=\iint_{0}^{\infty} g(w x) g(w y) W_{2 \tau}(x, y, p) d x d y$.

Остальные обозначения те же, что и в формуле (2.4.7) п.4.

Если последовательность имеет детерминированный тактовый интервал, то формула (2.4.13) п.5 переходит в

$\mathrm{F}(\mathrm{w})=$

$$
\begin{gathered}
\frac{2}{\mathrm{~T}}|\mathrm{x}(\mathrm{w}, \alpha)|^{2}\left\{\left(\mathrm{a}^{2}+\delta^{2}\right) \mathrm{K}^{0}(\mathrm{w})-\mathrm{a}^{2}\left|\theta_{1 \gamma} \mathrm{w}\right|^{2} \mathrm{~K}_{\infty}(\mathrm{w})+\psi(w)+\right. \\
\left.\mathrm{a}^{2}\left|\theta_{1 \gamma}(\mathrm{w})\right|^{2} \mathrm{~K}_{\infty}(\mathrm{w}) \frac{2 \pi}{\mathrm{T}} \sum_{\mathrm{r}=-\infty}^{\infty} \delta\left(w-\frac{2 \pi \mathrm{R}}{\mathrm{T}}\right)\right\}
\end{gathered}
$$

Здесь

$$
K_{\infty}(w)=\left|\int_{0}^{\infty} g(w x) W_{1 \tau}(x) d x\right|^{2}, \quad \psi(w)
$$

определяется по (2.4.10).

Рассмотрим последовательность равноотстоящих импульсов заданной формы, имеющих одинаковую амплитуду и случайную длительность (рис. 2.4). Так как в рассматриваемом случае $\delta=0, \theta_{1 \gamma}(\omega) \equiv 1$, то из (2.7.11) находим:

$F(w)=\frac{2 a^{2}}{T}|x(w, \alpha)|^{2}\left\{K_{0}(w)-K_{\infty}(w)+\psi_{3}+\frac{2 \pi}{T} K_{\infty}(w) \sum_{r \rightarrow-\infty}^{\infty} \delta\left(\omega-\frac{2 \pi R}{T}\right)\right\}$

где

$$
\begin{gathered}
\psi_{3}(w)=\lim _{N \rightarrow \infty} 2 \sum_{P=1}^{2 N}\left(1-\frac{P}{2 N+1}\right) x \\
x R_{e}\left\{\left(K_{P}(w)-K_{\infty}(w)\right) e^{-i \omega p T}\right\}
\end{gathered}
$$

функция $K_{P}(w)$ определяется по формуле (2.7.10). функции $K_{0}(w)$ и $K_{\infty}(w)$ получаются из $K_{P}(w)$ предельным переходом при $p \rightarrow 0$ и $p \rightarrow \infty$. Если случайные длительности любой пары импульсов взаимно независимы, то 


$$
\begin{gathered}
K_{p}(w)=K_{\infty}(w) \\
\text { и } \quad \psi_{3}(\omega)=0 .
\end{gathered}
$$

Тогда

$$
F(\omega)=\frac{2 a^{2}}{T}|\chi(\omega, \alpha)|^{2}\left[K_{0}(\omega)-K_{\infty}(\omega)+\frac{2 \pi}{T} K_{\infty}(\omega) \sum_{r=-\infty}^{\infty} \delta\left(\omega-\frac{2 \pi r}{T}\right)\right]
$$

Определим $K_{0}(w)$ и $K_{\infty}(w)$.

$$
\begin{aligned}
K_{P}(w)=\iint_{0}^{\infty} g(w x) g(w y) W_{2 \tau}(x, y, p) d x d y= \\
\quad=\iint_{0}^{\infty}\left(1-e^{-i w x}\right)\left(1-e^{-i w y}\right) W_{2 \tau}(x, y, p) d x d y .
\end{aligned}
$$

Вместо случайных величин $\mathrm{x}$ и $\mathrm{y}$ введем в рассмотрение $x^{\prime}=x-\tau_{0}$ и $y^{\prime}=y-\tau_{0}$ - случайные отклонения длительностей импульсов от среднего значения $\tau_{0}$ и $W_{2 \tau}\left(x^{\prime}, y^{\prime}, p T\right)$ - двумерную функцию распределения отклонений длительности импульсов от среднего значения $\tau_{0}$. Тогда

$$
K_{p}(\omega)=\iint_{-\infty}^{\infty}\left(1-e^{-i \omega \tau_{0}+i \omega \tau}\right) W_{2 \tau}(x, y, p T) d x d y=1+\theta_{2 \tau}(-\omega, \omega, p T)-e^{-i \omega \tau_{0}} \theta_{1 \tau}(-\omega) \text {, }
$$

где $\theta_{2 \tau}$ и $\theta_{1 \tau}$ - двумерная и одномерная характеристические функции случайного отклонения длительности импульса от средней длительности $\tau_{0}$. Из (2.7.14) предельным переходом находим:

$$
\begin{gathered}
K_{0}(\omega)=2-e^{-i \omega \tau_{0}} \theta_{1 \tau}(\omega)-e^{-i \omega \tau_{0}} \theta_{1 \tau(-\omega)}, \\
K_{\infty}(\omega)=1+\left|\theta_{1 \tau}(\omega)\right|^{2}-e^{i \omega \tau} \theta_{1 \tau}(\omega)-e^{i \omega \tau_{0}} \theta_{1 \tau}(-\omega)
\end{gathered}
$$

При независимости импульсов непрерывная часть спектра в соответствии с (2.7.13) имеет вид

$$
F_{H}(\omega)=\frac{2 a^{2}}{T}|\chi(\omega, \alpha)|^{2}\left[1-\left|\theta_{1 \tau}(\omega)\right|^{2}\right]
$$

и дискретная

$$
F_{g}(\omega)=\frac{4 \pi a^{2}}{T \omega^{2}}|\chi(\omega, \alpha)|^{2}\left[1+\mid \theta_{1 \tau}(\omega)^{2}-e^{i \omega \tau} \theta_{1 \tau}(\omega)-e^{i \omega \tau_{0}} \theta_{1 \tau}(-\omega)\right] \sum_{r=-\infty}^{\infty} \delta\left(\omega-\frac{2 \pi r}{T}\right)
$$


Если случайные отклонения длительности импульса от среднего $\tau_{0}$ подчиняются равномерному закону, т.е. отклонения $\Delta \tau=\tau-\tau_{0}$ могут принимать с равной вероятностью значения от $-\beta T$ до $\beta T$, то

$$
\begin{gathered}
\theta_{1 \tau}(\omega)=\int_{-\beta T}^{\beta T} e^{i \omega t} W_{1 \tau}(x) d x=\frac{1}{2 \beta T} \int_{-\beta T}^{\beta T} e^{i \omega t} d x=\frac{\sin \beta \omega T}{\beta \omega T} \\
F(\omega)=\frac{2 a^{2}}{T \omega^{2}} \frac{1}{1+\frac{\omega^{2}}{\alpha^{2}}}\left\{1-\frac{\sin \beta \omega T}{(\beta \omega T)^{2}}+\frac{2 \pi}{T}\left[1-2 \frac{\sin \beta \omega T}{\beta \omega T} \cos \omega \tau_{0}+\frac{\sin ^{2} \beta \omega T}{(\beta \omega T)^{2}}\right]_{r=-\infty}^{\infty} \delta\left(\omega-\frac{2 \pi r}{T}\right)\right\}
\end{gathered}
$$

Графики зависимостей

$$
\frac{F_{H(\omega)}}{F_{H}(0)}=\frac{12}{\left(\omega \tau_{0}\right)} \frac{1}{1+\frac{\omega^{2}}{a^{2}}}\left[1-\frac{\sin ^{2} \frac{\omega \tau_{0}}{2}}{\left(\frac{\omega \tau_{0}}{2}\right)^{2}}\right]
$$

и

$$
\frac{F_{g}(\omega)}{F_{g}(0)}=\frac{1}{\left(\omega \tau_{0}\right)^{2}} \frac{1}{1+\frac{\omega^{2}}{\alpha^{2}}}\left[1-2 \frac{\sin \frac{\omega \tau_{0}}{2}}{\frac{\omega \tau_{0}}{2}} \cos \omega \tau_{0}+\frac{\sin ^{2} \frac{\omega \tau_{0}}{2}}{\left(\frac{\omega \tau_{0}}{2}\right)}\right] \sum_{r=-\infty}^{\infty} \zeta\left(\omega-\frac{2 \pi r}{T}\right),
$$

от $\frac{\omega \tau_{0}}{2}$ при $\beta=\frac{1}{2} \frac{\tau_{0}}{T}, \frac{\tau_{0}}{T}=\frac{1}{5}$ приведены на рис. 2.5 для $\beta \tau_{0}=\infty$ и на рис.2.6 для $\beta \tau_{0}=5,4$.Сплошные кривые на этих и последующих графиках соответствуют непрерывному спектру.

Когда случайные отклонения длительности импульса от $\tau_{0}$ подчиняются нормальному закону с дисперсией $\delta_{\tau}^{2}$ и коэффициентом корреляции $R_{p, \tau}$, получаем [I] :

$$
\begin{gathered}
\theta_{1 \tau}(\omega)=e^{-\delta_{\tau \omega^{2}}^{2}}, \theta_{2 \tau}\left(\omega_{1} \omega_{2, \tau}\right)=e^{-\frac{\delta_{\tau}^{2}}{2}}\left(\omega_{1}^{2}+2 R_{p \tau} \omega_{1} \omega_{2}+\omega_{2}^{2}\right), \\
K_{p}(\omega)=1+e^{-\delta_{\tau \omega^{2}}^{2}\left(1-R_{p}\right)}-2 e^{-\frac{\delta_{\tau}^{2} \omega^{2}}{2}} \cos \omega \tau_{0} \\
K_{0}(\omega)=2\left(1-e^{-\frac{\delta_{\tau \omega^{2}}^{2}}{2}} \cos \omega \tau_{0}\right) \\
\mathrm{K}_{\infty}(\omega)=1+e^{-\delta_{\tau}^{2} \omega^{2}}-2 e^{-\frac{\delta_{\tau}^{2} \omega^{2}}{2}} \cos \omega \tau_{0}, \\
\psi_{3}=\lim _{N \rightarrow \infty} 2 \sum_{P=1}^{2 N}\left(1-\frac{P}{2 N+1}\right) e^{-\delta_{\tau}^{2} \omega^{2}}\left(e^{R_{p \tau} \delta_{\tau}^{2} \omega^{2}}-1\right) \cos p \omega T
\end{gathered}
$$

Если ряд (2.7.20) сходится, то 


$$
\psi_{3}(\omega)=2 e^{-\delta_{\tau}^{2} \omega^{2} \sum_{p=1}^{\infty}\left(e^{R_{p \tau} \delta_{\tau}^{2} \omega^{2}}-1\right) \cos p \omega T}
$$

Разлагая экспоненциальную функцию в ряд, меняя порядок суммирования и обозначая

$$
\psi_{3}(\omega)=e^{-\delta_{\tau}^{2} \omega^{2}} \sum_{k=1}^{\infty} R_{p \tau}^{k} \cos p \omega T
$$

получим

$$
\psi_{3}(\omega)=e^{-\delta_{\tau}^{2} \omega^{2}} \sum_{k=1}^{\infty} \frac{\delta_{\tau}^{2} \omega^{2 k}}{K !} \psi_{3 K}(\omega)
$$

и

$$
F(\omega)=\frac{2 a^{2}}{T \omega^{2}} \frac{1}{1+\frac{\omega^{2}}{\alpha^{2}}}\left\{1-e^{-\delta_{\tau}^{2} \omega^{2}}+e^{-\delta_{\tau}^{2} \omega^{2}} \sum_{k=1}^{\infty} \frac{\delta_{\tau}^{2 k} \omega^{2 k}}{K !} \psi_{3 K}(\omega)+\frac{2 \pi}{T}\left(1+e^{-\delta_{\tau}^{2} \omega^{2}}-2 e^{\frac{\delta_{\tau}^{2} \omega^{2}}{2}} \cos \tau_{0} \sum_{r=-\infty}^{\infty} \delta\left(\omega-\frac{2 \pi r}{T}\right)\right)\right\}
$$

-энергетический спектр последовательности импульсов (рис.2.3.) при модуляции нормальным шумом.

При модуляции белым шумом сумма по К отсутствует, так как при этом $\psi_{3 K} \equiv 0$.

При модуляции белым шумом непреерывная часть спектра, как следует из (2.7.21), имеет вид

$$
F_{H}(\omega)=\frac{2 a^{2}}{T \omega^{2}} \frac{1}{1+\frac{\omega^{2}}{\alpha^{2}}}\left(1-e^{-\delta_{\tau}^{2} \omega^{2}}\right)
$$

и дискретная -

$$
F_{g}(\omega)=\frac{4 \pi a^{2}}{T \omega^{2}} \frac{1}{1+\frac{\omega^{2}}{\alpha^{2}}}\left(1+e^{-\delta_{\tau}^{2} \omega^{2}}-2 e^{\frac{\delta_{\tau}^{2} \omega^{2}}{2}} \cos \tau_{0}\right) \sum_{r=-\infty}^{\infty} \delta\left(\omega-\frac{2 \pi r}{T}\right)
$$

На рис.2.7, 2.8 приведены графики зависимостей

$$
\frac{F_{H}(\omega)}{F_{H}(0)}=\frac{1}{\omega^{2} \tau_{0}^{2}} \frac{1}{1+\frac{\omega^{2}}{\alpha^{2}}}\left(1-e^{-\delta_{\tau}^{2} \omega^{2}}\right)
$$

И

$$
\frac{F_{g}(\omega)}{F_{g}(0)}=\frac{1}{\omega^{2} \tau_{0}^{2}} \frac{1}{1+\frac{\omega^{2}}{\alpha^{2}}}\left(1+e^{-\delta_{\tau}^{2} \omega^{2}}-2 e^{\frac{\delta_{\tau}^{2} \omega^{2}}{2}} \cos \omega \tau_{0}\right) \sum_{r=-\infty}^{\infty} \delta\left(\omega-\frac{2 \pi r}{T}\right)
$$

от $\omega \tau_{0}$ при $\delta_{\tau}=0,1 \tau_{0}, \tau_{0} / T=1 / 10$ для $\alpha \tau_{0}=\infty\left(\right.$ рис.2.7) и для $\alpha \tau_{0}=5,4($ рис.2.8) 
Пусть импульсы последовательности (рис.2.3) возникают в случайные моменты времени. Ограничимся случаем, когда длительности импульса и паузы независимы. Исходя из формулы (2.7.5), так же, как в п.6, нетрудно получить следующее выражение для энергетического спектра:

$$
F(\omega)=\frac{2 a^{2}}{T} \chi \chi(\omega, \alpha)^{2}\left\{\left(1+\frac{\delta^{2}}{\alpha^{2}}\right) K_{0}(\omega)+2 R_{e}\left[\frac{Q(-\omega) Q_{1}(-\omega) \theta_{1 \tau}(\omega)}{1-\theta_{1 \mu}(\omega)}\right]+\frac{g^{2}(0)}{T} \delta_{(\omega)}\right\},
$$

где

$$
\begin{aligned}
& Q(\omega)=m_{1}\left\{g\left(\omega \tau_{n}^{(k)}\right)\right\}=\int_{0}^{\infty} g(\omega x) W_{1 \tau}(x) d x, \\
& Q_{1}(\omega)=m_{1}\left\{g_{1}\left(\omega \tau_{n}^{(k)}\right)\right\}=\int_{0}^{\infty} g_{1}(\omega x) W_{1 \tau}(x) d x, \\
& g_{1}\left(\omega \tau_{n}^{(k)}\right)=e^{-i \omega \tau_{n}^{(k)}}-1, \quad g\left(\omega \tau_{n}^{(k)}\right)=1-e^{-i \omega \tau_{n}^{(k)}},
\end{aligned}
$$

$\mathrm{K}_{0}(\omega), \theta_{1 \mu}(\omega)$ определяются соответственно по формулам (2.7.9) и (2.6.2). Если длительность импульсов постоянна, то

$$
\begin{gathered}
W_{1 \tau}(x)=\int\left(x-\tau_{0}\right) \\
Q(\omega)=\int_{0}^{\infty} g(\omega x) w_{1 \tau}(x) d x=\int_{0}^{\infty} g(\omega x) \int\left(x-\tau_{0}\right) d x=g\left(\omega \tau_{0}\right) \\
Q_{1}(\omega)=g_{1}\left(\omega \tau_{0}\right), \theta_{1 \tau}(\omega)=e^{i \omega \tau_{0}}, \theta_{1 \mu}(\omega)=\theta_{1 \tau}(\omega) e^{i \omega \tau_{0}}, K_{0}(\omega)=\left|g\left(\omega \tau_{0}\right)\right|^{2}
\end{gathered}
$$

и (2.7.26) переходит в

$$
\begin{aligned}
& F(\omega)=\frac{2 a^{2}}{T}|x(\omega, \alpha)|^{2}\left\{\left[1+\left(\frac{\sigma}{\alpha}\right)^{2}\right]\left|g\left(\omega \tau_{0}\right)\right|^{2}+2 \operatorname{Re}\left[\frac{g\left(-\omega \tau_{0}\right) g_{1}\left(-\omega \tau_{0}\right) \theta_{1 \mu}(\omega) e^{-i \omega \tau_{0}}}{1-\theta_{1 \mu}(\omega)}\right]+\right. \\
& \left.\frac{g^{2}(0)}{T} S(\omega)\right\}
\end{aligned}
$$

Выражение, (2.7.27) после несложных преобразований приводится к более простому виду:

$$
F(\omega)=\frac{2 a^{2}}{T}|x(\omega \alpha)|^{2}\left|g\left(\omega \tau_{0}\right)\right|^{2}\left\{\left(\frac{\sigma}{a}\right)^{2}+\frac{1-\left|\theta_{1 \mu}(\omega)\right|^{2}}{1-2 \operatorname{Re} \theta_{1 \mu}(\omega)+\left|\theta_{1 \mu}(\omega)\right|^{2}}+\frac{1}{T}\right\}
$$

Применим формулу (2.7.28) для двух случаев.

1/ Паузы между последовательными импульсами распределены по экспоненциальному закону

$$
W_{1 \tau}(t)=\frac{1}{T_{1}} e^{-\frac{t}{T_{1}}} T_{1}=T-\tau_{0} t>0
$$

В (2.7.29) $T_{1}$-среднее значение паузы между импульсами.

$$
\theta_{1 \tau} *(\omega)=\int_{0}^{\infty} e^{i w x} W_{1 \tau}(x) d x=\frac{1}{T} \int_{0}^{\infty} e^{i w x-\frac{x}{T}} d x=\frac{1}{1-i w T_{1}}
$$




$$
\begin{aligned}
\theta_{1 \mu}(w) & =\frac{e^{i w \tau}}{1-i w \tau}\left|\theta_{1 \mu}(w)\right|^{2}=\frac{1}{1+w^{2} T^{2}} \\
\operatorname{Re} \theta_{1 \mu}(w) & =\frac{\cos w \tau_{0}-w T_{1} \sin w \tau_{0}}{1+w^{2} T^{2}}
\end{aligned}
$$

Подставив (2.7.30) в (2.7.28) и положив $\sigma=0$ получим

$$
\begin{aligned}
F(w)= & \frac{2 a^{2}}{T}|x(w \alpha)|^{2}|g(w \tau)|^{2}\left[\frac{1}{1+2 \frac{\sin w \tau}{w T}+\left(\frac{\sin \frac{W \tau}{2}}{\frac{W T}{2}}\right)}+\frac{1}{T} s(w)\right]= \\
& \frac{8 a^{2}}{T W^{2}} \frac{\sin ^{2} \frac{w \tau_{0}}{2}}{1+\frac{W^{2}}{\alpha^{2}}} \frac{1}{1+\frac{\sin w \tau_{0}}{W T_{1}}+\left(\frac{\sin \frac{w \tau_{0}}{T_{2}}}{\frac{w W}{2}}\right)^{2}}+\frac{2 a^{2} \tau_{0}^{2}}{T^{2}} s(w)
\end{aligned}
$$

На рис. 2.9 представлен график зависимости

$$
\frac{F_{H}(w)}{F_{H}(0)}=\frac{16}{w^{2} \tau_{0}^{2}} \frac{1}{1+\frac{w^{2}}{\alpha^{2}}} \frac{\sin ^{2} \frac{w \tau_{0}}{2}}{1+2 \frac{\sin w \tau_{0}}{w \tau_{0}}+\left(\frac{\sin \frac{w \tau_{0}}{2}}{\frac{w}{2}}\right)^{2}}
$$

От $\omega \tau_{0}$ для значений $\alpha \tau_{0}=\infty$ и $\alpha \tau_{0}=5.4$. В (2.7.32) принято $\tau_{0}=\frac{T}{2}, T_{1}=\tau_{0}$

2/ Паузы между импульсами распределены нормально со средним $T_{1}=T-\tau_{0}$ и дисперсией $\sigma_{\mu}^{2} / \sigma_{\mu} \ll T$. Тогда

$$
\begin{gathered}
\theta_{1 \tau} *(\omega)=e^{i w T_{1}-\frac{\sigma_{\mu}^{2} \omega^{2}}{2}} \theta_{1 \mu}(\omega)=e^{i w T-\frac{\sigma_{\mu}^{2} w^{2}}{2}} \\
\left|\theta_{1 \mu}(w)\right|^{2}=e^{-\sigma_{\mu}^{2} \omega^{2}} \operatorname{Re}_{1 \mu}(\omega)=e^{\frac{-\sigma_{\mu}^{2} w^{2}}{2}} \cos w T
\end{gathered}
$$

И из (2.7.28) находим $/ \sigma=0 /$ :

$$
F(w)=\frac{8 a^{2}}{T W^{2}} \frac{1}{1+\frac{w^{2}}{\alpha^{2}}} \sin ^{2} \frac{w \tau_{0}}{2}\left[\frac{1-e^{-\sigma_{\mu}^{2} \omega^{2}}}{1-e^{-\frac{\sigma_{\mu}^{2} w^{2}}{2}} \cos w T+e^{-\sigma_{\mu}^{2} w^{2}}}+\frac{1}{T} s(w)\right]
$$

На рис. 2.10 изображены графики зависимости $\lg \frac{F_{H}(w)}{F_{H}(0)}$

$$
\frac{F_{H}(w)}{F_{H}(0)}=\frac{T^{2}}{\sigma_{\mu}^{2}} \frac{1}{1+\frac{W^{2}}{\alpha^{2}}} \frac{\sin ^{2} \frac{w \tau}{2}}{\left(\frac{W \tau}{2}\right)} \frac{1-e^{-\sigma_{\mu}^{2} \omega^{2}}}{1-2 e^{-\frac{\sigma_{\mu}^{2} \omega^{2}}{2}} \cos w T+e^{-\sigma_{\mu}^{2}}}
$$

от wТ при $\sigma_{\mu}=0.1 T$ и $\tau_{0}=0.11$ для $\alpha \tau_{0}=\infty$ и $\alpha \tau_{0}=5.4$. 


\subsection{1 Последовательность экспоненциальных импульсов /детерминированный тактовый интервал/}

Исходим из общей формулы (2.5.6):

$$
\begin{array}{r}
F(w)=\frac{2}{T}\left\{\left(a^{2}+\sigma^{2}\right) K_{0}(w)-a^{2}\left|\theta_{1 \gamma}(w)\right|^{2} K_{\infty}(w)+\psi(w)\right. \\
\left.+a^{2}\left|\theta_{1 \gamma}(w)\right|^{2} K_{\infty}(w) \frac{2 \pi}{T} \sum_{r=-\infty}^{\infty} s\left(w-\frac{2 \pi r}{T}\right)\right\}
\end{array}
$$

Для импульсов неограниченной длительности

$$
K_{0}(w)=K_{\infty}(w)=(g(w))^{2} g(w)=\int_{0}^{\infty} u(t) e^{-i \omega t} d t
$$

Если кроме того считать импульсов взаоимно независымыми а амплитуду импульсов детерминированной то $\psi(\omega)=0, \sigma=0$ и формула (2.5.6) упрощается

$$
F(w)=\frac{2 a^{2}}{T}|g(w)|^{2}\left\{1-\left|\theta_{1 \gamma}(w)\right|^{2}+\left|\theta_{1 \gamma}(\omega)\right|^{2} \frac{2 \pi}{T} \sum_{r=-\infty}^{\infty} s\left(w-\frac{2 \pi r}{T}\right)\right\}
$$

Для последовательности экспоненциальных импульсов (рис. 2.11)

$$
\begin{gathered}
U(t)=e^{-\alpha t}, t>0 \\
\mathrm{~g}(\mathrm{w})=\int_{0}^{\infty} \mathrm{e}^{-\alpha \mathrm{t}-\mathrm{i} \omega \mathrm{t}} \mathrm{dt}=\frac{1}{\alpha+\mathrm{iw}}
\end{gathered}
$$

При равномерном распределений шума на интервале от $-\beta \mathrm{T}$ до $\beta \mathrm{T}$, как было показано, $\theta_{1 \gamma}(\omega)=\frac{\sin \beta w T}{\beta w T}$ и (2.7.1.1)

Переходит в

$$
F(w)=\frac{2 a^{2}}{T W^{2}} \frac{1}{1+\frac{w^{2}}{\alpha^{2}}}\left\{1-\frac{\sin ^{2} \beta w T}{(\beta w T)^{2}}+\frac{\sin ^{2} \beta w T}{(\beta w T)^{2}} \frac{2 \pi}{T} \sum_{r=-\infty}^{\infty} s\left(w-\frac{2 \pi r}{T}\right)\right\}
$$

Графики зависимости

$$
\frac{F_{H}(w)}{F_{H}(0)}=\frac{3}{(\beta w T)^{2}} \frac{1}{1+\frac{w^{2}}{\alpha^{2}}}\left[1-\frac{\sin ^{2} \beta w T}{(\beta w T)^{2}}\right]
$$

И

$$
\frac{F_{g}(w)}{F_{g}(0)}=\frac{1}{1+\frac{w^{2}}{\alpha^{2}}} \frac{\sin ^{2} \beta w T}{(\beta w T)^{2}} \sum_{r=-\infty}^{\infty} s\left(w-\frac{2 \pi r}{T}\right)
$$


От $\frac{\omega \tau}{10}$ при $\beta=\frac{1}{10}$ для $\alpha T=10^{-3}$ представлены на рис. 2.12 и для $\alpha T=10$ - на рис. 2.13.

\subsection{2 Последовательность экспоненциальных}

\section{импульсов, возникающих в случайные моменты времени}

Для нахождения спектра в этом случае следует воспользоваться формулой (2.66), которая для детерминированной амплитуды импульсов $/ \sigma=0 /$ принимает вид:

$$
F(w)=\frac{2 a^{2}}{T W^{2}} \frac{1}{1+\frac{w^{2}}{\alpha^{2}}}\left\{1+2 \operatorname{Re} \frac{\theta_{1 \mu}(w)}{1-\theta_{1 \mu}(w)}+\frac{1}{T} s(w)\right\}
$$

Пусть паузы между импульсами распределены нормально со средним $\mathrm{T}$ и дисперсией $\sigma_{\mu}^{2} / \sigma_{\mu} \ll \mathrm{T} /$. Тогда

$$
\left.\begin{array}{c}
\theta_{1 \mu}(w)=e^{i \omega t--\frac{\sigma_{\mu}^{2} \omega^{2}}{2}} \\
\operatorname{Re} \frac{\theta_{1 \mu}(w)}{1-\theta_{1 \mu}(w)}=e^{--\frac{\sigma_{\mu}^{2} \omega^{2}}{2}} \frac{\cos w T-e^{-\frac{\sigma_{\mu}^{2} \omega^{2}}{2}}}{1-2 e^{-\frac{\sigma_{\mu}^{2} \omega^{2}}{2}} \cos w T+e^{-\frac{\sigma_{\mu}^{2} \omega^{2}}{2}}} \\
F(w)=\frac{2 a^{2}}{T W^{2}} \frac{1}{1+\frac{W^{2}}{\alpha^{2}}}\left\{\frac{1-e^{-\frac{\sigma_{\mu}^{2} \omega^{2}}{2}}}{1-2 e^{-\frac{\sigma_{\mu}^{2} \omega^{2}}{2}} \cos w T+e^{-\frac{\sigma_{\mu}^{2} \omega^{2}}{2}}}+\frac{1}{T} s(w)\right.
\end{array}\right\}
$$

На рис. 2.14 приведены графики зависимости $\lg \frac{F_{H}(w)}{F_{H}(0)}$

$$
\frac{F_{H}(w)}{F_{H}(0)}=\frac{T^{2}}{\sigma_{\mu}^{2}} \frac{1}{1+\frac{w^{2}}{\alpha^{2}}} \frac{1-e^{-\frac{\sigma_{\mu}^{2} \omega^{2}}{2}}}{-2 e^{-\frac{\sigma_{\mu}^{2} \omega^{2}}{2}} \cos w T+e^{-\frac{\sigma_{\mu}^{2} \omega^{2}}{2}}}
$$

От шТ при $\sigma_{\mu}=0.1 T$ для $\alpha T=10^{3}$ и $\alpha T=10$. 


\section{ГЛАВА 3 \\ ОСОБЕННОСТИ ФУНКЦИОНИРОВАНИЯ ЛАЗЕРНЫХ \\ ИЗМЕРИТЕЛЬНЫХ СИСТЕМ В КАНАЛАХ СО СЛУЧАЙНЫМИ ПАРАМЕТРАМИ}

\section{1Постановка задачи}

Высокие потенциальные возможности лазерных измерительных систем (ЛИС), обусловленные прежде всего высокими точно-устными характеристиками, в значительной степени ограничиваются условиями распространения световых волн в реальных материальных средах, в частности в атмосфере (рис. 3.1).

Наибольшее влияние на оптический измерительный канал оказывает экранирующее действие облачных неоднородностей, которые характеризуются коэффициентами ослабления в десятки и сотни децибел на километр и значительными пространственными и временными масштабами [2-7]. Внутренний пространственный масштаб - размер облаков и облачных образований колеблется от 10 м до 10 км, а внешний, характеризующий размер поля, достигает сотен и даже тысяч километров [3-5]. Время жизни полей облачных неоднородностей составляет от нескольких часов до нескольких суток, а отдельных облачных образований - от десятков до сотен минут [57]. Значительно меньшими величинами ослабления, а также пространственных и временных масштабов характеризуются аэрозольные поля [1].

В условиях прозрачной атмосферы, когда облачные и аэрозольные поля отсутствуют, определяющим становится влияние мультипликативных помех, обусловленных рассеянием оптического сигнала на турбулентных неоднородностях различного масштаба. Внутренний $l_{0}$ и внешний $L_{0}$ масштабы турбулентных неоднородностей составляют примерно 1 мм и $1 \ldots$ 100 м, а время жизни неоднородностей, соизмеримых с $l_{0}$, достигает единиц миллисекунд [8, 9].

Наиболее сильно влияние атмосферы проявляется в протяженных оптических каналах, например космос - Земля и Земля - космос, которые используются для траекторных и астрономических измерений, локации Луны, решения калибровочных и юстировочных задач [10-13]. Использование ЛИС в этих каналах требует учета особенностей распространения световых волн, которые обеспечивают оптический контакт с ИСЗ в пределах пространства над горизонтом наблюдателя. Если к этому добавить малую продолжительность сеанса измерений из-за высокой скорости перемещения ИСЗ и низкую точность измерений из одного пункта, то вполне естественным окажется использование совокупности ЛИС, рассредоточенных на обширной территории и образующих измерительный комплекс.

Таким образом, в отличие от радиотехнических космических измерительных комплексов [14], в которых выбором диапазона длин волн удается существенно снизить мешающее действие атмосферных образований, 
эффективность применения ЛИС в значительной степени определяется как геометрией их расположения и динамикой движения ИСЗ (что сближает их с радиотехническими измерителями), так и статистическими характеристиками полей атмосферы. Эти характеристики играют основную роль при синтезе структуры измерительных комплексов, используемых при локации медленно перемещающихся объектов (Луна стационарные ИЗС) $[11,12]$.

В зависимости от масштабов атмосферных неоднородностей и пространственно-временных характеристик их полей проблема повышения эффективности ЛИС должна решаться на разных иерархических уровнях.

Первый уровень предусматривает адаптацию структуры ЛИС к возмущениям атмосферы [15] или целенаправленное изменение возмущений [16], выбор оптимальных параметров измерительной системы, комплексирование оптических и радиотехнических измерителей [13]. Этот уровень несет в значительной степени отпечаток индивидуальных свойств ЛИС, анализ которых дан в гл. 2.

Второй уровень, являющийся определяющим, связан с синтезом пространственно-временной структуры комплекса ЛИС, оптимально согласованной со стохастической структурой облачных полей и динамикой движения ИСЗ [17]. Комплекс ЛИС обладает всеми признаками больших систем: целенаправленностью и вероятностным характером функционирования, иерархичностью структуры, сложными переплетающимися связями и возможностью адаптации к внешним условиям [18]. Поэтому решение задач анализа и синтеза структуры комплекса ЛИС и выбора целесообразных стратегий его применения должно производиться с позиций системного подхода. Основой системного подхода служит описание рассматриваемой системы (в данном случае комплекса ЛИС) на различных уровнях абстракции, чтобы наиболее простое описание отражало основные аспекты поведения системы. Для синтеза пространственно-временной структуры комплекса ЛИС целесообразно задавать общую и частную модели его функционирования. Общая модель должна включать частные модели, характеризующие основные процессы функционирования комплекса пространственно-разнесенных ЛИС.

\section{2 Математическая модель комплекса пространственно- разнесенных лис}

Общая абстрактная модель функционирования сложной системы, при задании ее входов и выходов в теории систем рассматривается как отношение декартова произведения серии множеств [18]:

$$
S \subseteq X \times Y
$$

где $X=X_{1} \times X_{2} \times \ldots \times X_{n}-$ множество входов, состоящее из подмножеств $X_{i} ; \mathrm{y}-$ множество выходов системы.

Если $S$ - функция, то комплекс ЛИС - функциональная система, представляющая собой отображение абстрактного множества $X$ в абстрактное 
множество $Y$, т. е. $S: X \rightarrow Y$, которое каждому элементу: $x \in X$ ставит в соответствие единственный элемент из $Y$.

Обоснование моделей комплекса ЛИС, соответствующих различным уровням описания, и выбор исходных множеств, определяемых соотношением (1.1), необходимо производить на основе задач, решаемых суперсистемой, с учетом характерных свойств ЛИС и облачных полей.

Комплекс ЛИС можно представить в виде двухуровневой системы $S_{1}, S_{2}$ (рис. 3.2), где верхний уровень (измерительный комплекс) представляет ярко выраженную пространственно-временную систему. Особенностью рассматриваемой модели является включение на различных иерархических уровнях моделей возмущающих полей с разномасштабными неоднородностями. Входами системы верхнего уровня являются модель информационного оптического поля, обусловленного взаимодействием с исследуемым объектом, а также модель облачных полей макромасштаба.

Наиболее отвечающей современным представлениям является модель космического измерительного комплекса в виде пространственно-временного фильтра, позволяющего оценить параметры исследуемого материального объекта, которые содержатся в параметрах оптического информационного поля $[19,20]$. Однако при исследовании предельных возможностей (потенциальной эффективности) комплекса, связанных только с характеристиками облачных полей, представляется целесообразным использовать более простые модели. Систему верхнего уровня будем описывать множеством $N$, элементами которого является количество пунктов и координаты их размещения, и множеством способов использования $C$, элементы которого позволяют составить пространственно-временные алгоритмы применения и взаимодействия средств комплекса. Система нижнего уровня комплекса ЛИС полностью определяется показателями эффективности, которые образуют множество $L$ в формализованном описании системы.

Выходные характеристики комплекса ЛИС определяют эффективность его функционирования в интересах суперсистемы и составляют множество $P$, элементами которого является группа вероятностных показателей эффективности. Таким образом, комплекс ЛИС математически описывается как отношение над следующими множествами: $S \subseteq Q \times \Omega \times L \times N \times C \times R \times T \times P$, где $R$ множество точек некоторой ограниченной области пространства, значительно превышающей интервалы корреляции облачных полей; $T$ - множество моментов времени; $\Omega$ — множество характеризующее пропускание облачных полей.

Очевидно, что полная абстрактная модель комплекса ЛИС сложна, поэтому использование ее даже для анализа сопряжено с серьезными трудностями. Введем упрощающие предположения о непрерывном существовании информационного поля над заданной территорией и об отсутствии потока отказов ЛИС, т. е. принимаем $Q=\{1\}$ и $L=\{1\}$, что является правомерным при оценке потенциальной эффективности комплекса ЛИС. Для того чтобы получить функциональную зависимость между элементами 
множеств $\Omega, N, C$ и $P$ будем рассматривать облачные поля на интервалах $\Delta R_{i}$ и $\Delta T_{i}$, отвечающих условиям квазистационарности и пространственной однородности, т. е.

$$
\Omega_{R, T}=\Omega \times R \times T=\Omega_{\Delta R_{1}, \Delta T_{1}} \times \ldots \times \Omega_{\Delta R i, \Delta T i} \times \ldots \times \Omega_{\Delta R n, \Delta T_{n}} .
$$

Интервалы пространственной однородности статистических характеристик облачных полей, определяемые мезо- и макромасштабными атмосферными процессами, изменяются от нескольких сотен до нескольких тысяч километров $[5,21,22]$. Если подмножества $\Omega_{\triangle R i, \Delta T i}$ представляют усредненные характеристики пропускания на определенных пространственных и временных интервалах, можно получить квазидетерминированное описание системы (комплекса ЛИС) в виде отображения $g: \Omega_{R, T} \times N \times C \rightarrow P$, где $g$ некоторая функция, связывающая однозначно элементы $\omega, n, c(\omega \in \Omega ; n \in N ; c \in C)$ с элементом $p \in P$, т. е. $p=g(\omega, n, c)$. Тогда задача синтеза пространственно-временной структуры измерительного комплекса сводится к задаче такого размещения ЛИС и выбору стратегии его применения, которые были бы оптимальными в смысле некоторых показателей и критериев эффективности при задании характеристик облачных полей $\Omega_{\triangle R i, \Delta T i}$. Задача оптимального размещения ЛИС (при заданной стратегии) должна конкретизироваться заданием подмножества $N_{C}^{M} \times \Omega_{R, T} \leq N_{c, \Omega}^{Д}$, являющегося подмножеством допустимых решений множества $N_{C} \times \Omega_{R, T}$. При этом требуется найти такое $n^{*} \in N_{c, \Omega}^{\not}$, чтобы для всех $\omega$ из $\Omega_{R, T}$ выполнялось неравенство

$$
g\left(n_{\Omega}^{*}\right) \geq g(n)
$$

где $g(n)$ - показатель эффективности (целевая функция), а неравенство (1.2) служит критерием оптимальности.

Таким образом, для решения задачи оптимального размещения пунктов, оснащенных ЛИС, предварительно должен быть проведем анализ распределения облачных полей и их корреляционных характеристик на рассматриваемой территории я определенных временных интервалах, осуществлен выбор благоприятных районов размещения средств и выполнена оценка потенциальной эффективности комплекса пространственноразнесенных на основе моделей облачных полей и частной модели функционирования комплекса.

Районировать территорию можно по методике, изложенной в работе [21], на основе характеристик вероятности существования (повторяемости) облачных полей определенных градаций. В частности, в работе [21] проведено районирование территории США по характеру годового хода повторяемости облачных полей макромасштаба, которое позволяет выделить большие районы с квазиоднородными характеристиками облачных полей и выбрать наиболее благоприятные для размещения ЛИС.

Следующий этап состоит в определении интервалов корреляции облачных полей, детальном районировании выделенных квазиоднородных районов и выборе пунктов размещения ЛИС с учетом особенностей распределения возмущающих полей мезомасштаба (облачных и аэрозольных полей). 
Последние отражают влияние рельефа и местные особенности формирования облачности, туманов и других аэрозольных полей и, как показано в работе [22] при районировании территории Сев. Америки по характеру годового хода сплошной облачности и метеорологической дальности видимости, позволяют уточнить возможности и границы благоприятных районов. На этом этапе целесообразно осуществлять анализ эффективности применения комплекса ЛИС c использованием статистических моделей возмущающих полей атмосферы, и прежде всего пространственно- временных моделей облачных полей.

\section{3Модели полей облачных неоднородностей и вероятностные характеристики сеанса измерений}

Рассмотрим статистические характеристики пропускания двух основных типов полей - макро- и мезомасштаба, вертикальный разрез которых изображен на рис. 3.3. В качестве поля с дискретными облачными неоднородностями будем рассматривать поля кучевой облачности, которые являются наиболее распространенными среди полей мезомасштаба [5, 23]. Для определения среднего пропускания воспользуемся моделью пространственной структуры случайного поля [23], которая удовлетворительно описывает поле кучевой облачности в условиях следующих предположений: поле облачности однородное и изотропное, высота нижней границы всех облачных неоднородностей поля одинакова, атмосфера плоскопараллельна, облачные элементы аппроксимированы цилиндрами, центры которых распределены по закону Пуассона в плоскости $H=H_{a}$, толщина облачной неоднородности примерно равна ее диаметру $(h \approx d)$.

Из-за большой толщи облачного поля и значительных коэффициентов ослабления можно не учитывать все тонкости вертикальной структуры сплошного облачного поля или отдельной облачной неоднородности, поэтому в дальнейшем будем различать две градации пропускания $Z=\{0,1\}$, т. е. будем рассматривать облачные неоднородности как непрозрачные экраны. Очевидно, что пропускание облачного поля в заданном направлении зависит от пространственной структуры поля (количества и размеров облачных неоднородностей, их распределения в пространстве) и является случайной величиной.

В первом приближении связь между усредненным пропусканием облачного поля и его пространственной структурой может быть получена в предположении двумерности облачных неоднородностей на основании соотношения

$$
\bar{Z}_{a}=\frac{1}{A} \iint_{A} Z(x, y) d x d y,
$$

где $A$ - размер зоны видимости; $Z(x, y)=\{0,1\}$. Область интегрирования в (3.3.1) можно разделить на две части: для одной $Z=1$, для другой $Z=0$. Тогда величина $\bar{Z}_{a}$ будет характеризовать отношение прозрачного участка зоны видимости ко всей ее площади, т.е. вероятность того, что случайно 
расположенная зона видимости в облачном поле накроет участки с $Z=1$, и может служить оценкой вероятностных характеристик сеанса измерений. Для пуассоновского распределения центров облачных неоднородностей при $A \rightarrow \infty$ среднее пропускание поля [24]

$$
\bar{Z}_{a}=\exp \left\{-2 \pi \mu \int_{0}^{\infty}[1-F(r)] r d r\right\},
$$

где $\mu$ - плотность заполнения площади облачными центрами; $F(r)-$ функция распределения размеров облачных неоднородностей.

Если размеры всех облачных неоднородностей одинаковы, из (3.3.2) получим $\bar{Z}_{a}=\exp \left(-\mu \pi d^{2} / 4\right)$.

Полученная характеристика облачного поля, увеличенная в 10 раз, аналогична используемому в метеорологии абсолютному баллу $j_{a}$ и связана с ним как $\bar{Z}_{a} \approx 1-j_{\alpha} / 10$. Абсолютный балл $j_{a}$, характеризующий количество облачности в зените над данным пунктом, определяется долей облачных неоднородностей, спроектированных на воображаемую сферу с центром в центре Земли. Следовательно, усредненное пропускание $Z_{a}$ представляет вероятность проведения сеанса измерений параметров объекта, линия визирования на который неподвижна относительно облачных неоднородностей в течение времени измерений и проходит близко к зениту $(\xi \approx 0)$.

При произвольном направлении на объект необходимо учитывать трехмерность облачных неоднородностей. Тогда усредненное пропускание для изотропного облачного поля, которое соответствует принятому в метеорологии относительному баллу, будет определяться как [23]

$$
\bar{Z}_{a}=\int p(\xi) \sin \xi d \xi
$$

где

$$
p(\xi)=\exp \left(-\mu \pi d^{2} / 4+2 d h t g \xi\right)
$$

- вероятность свободной линии визирования в заданном направлении $\xi$ (вероятность оптического контакта с ИСЗ). В соотношении (3.3.3) параметры $\mu$, $d, h$ являются функцией количества облачности $j$.

Зависимость $p(\xi)$, рассчитанная на основании (3.3.3) для полей кучевой облачности, показана на рис. 3.4 [23]. Из рисунка видно, что для $2 \leq j \leq 6$ увеличением зенитного угла вероятность $p(\xi)$ уменьшается незначительно, пока $\xi \leq 50^{\circ}$, а после начинает сказываться трехмерность облачных неоднородностей и наблюдается резкий спад $p(\xi)$ по направлению к горизонту. Отсюда следует, что относительное пропускание $Z_{0}$ всегда меньше абсолютного. Для рассматриваемой модели кучевой облачности получена следующая эмпирическая формула, устанавливающая связь между относительным и абсолютным пропусканием [5, 23]: $\bar{Z}_{0}=\bar{Z}_{a}-0,5\left(1-\bar{Z}_{a}\right) \bar{Z}_{a}, \quad 0,4 \leq \bar{Z}_{a} \leq 0,9$.

Как уже подчеркивалось, величина $Z_{0}$ по существу представляет вероятность оптического контакта с ИСЗ в произвольном направлении, когда 
линия визирования за время сеанса связи неподвижна относительно облачных неоднородностей, т. е. $P_{c}=\bar{Z}_{0}=1-j / 10$, где $j$ - относительный балл, который определяется как доля закрытости облачными неоднородностями воображаемой полусферы с центром в пункте наблюдения. Относительный бал $j$, имеющий градации от 0 до 10 через один балл, получают при наземных наблюдениях, которые до появления спутниковой метеорологии были основными. На синоптические карты обычно наносятся и используются при обработке некоторые группировки градаций. Например, в [25] применена группировка градаций

$0 . .2,3 \ldots 7,8 \ldots 10$. Преимущество этой группировки заключается в том, что в первой и последующих градациях объединяются поровну четные и нечетные оценки облачности $(1,2)$ и $(8,9)$ и тем самым исключаются субъективные ошибки наблюдателя. В последнее время для обработки облачности стали привлекаться градации $0,1 \ldots .3,4 \ldots . .6,7 \ldots 9,10$, которые обладают преимуществами в статистическом отношении [26,27].

В общем случае, когда линия визирования перемещается относительно облачных неоднородностей (вследствие движения объекта или перемещения облачного поля в зоне видимости), главной составляющей качества сеанса, измерений становится время его проведения, т. е. сеанс должен рассматриваться как временной процесс. Тогда необходимым условием проведения сеанса измерений на заданном интервале $t_{6}$ является выполнение условия $\hat{t}_{6 \kappa}+\hat{t}_{c u} \leq \hat{t}_{n}$, которое выполняется с вероятностью

$$
P_{c}=\left(\hat{t}_{6 \kappa}+\hat{t}_{c u} \leq \hat{t}_{n} ; \hat{t}_{B}\right)
$$

Здесь $\hat{t}_{\text {- }}$ - продолжительность видимости ИСЗ в отсутствие облачных полей; $t_{\text {вк }}$ и $t_{\text {си }}$ - случайные длительности вхождения в контакт и сеанса измерений; $t_{n}$ - случайная продолжительность видимости объекта при прохождении им просветов между облачными неоднородностями. Определение вероятности $P_{c}(3.3 .4)$ представляет значительные трудности и в частном случае сводится к задаче о перекрытии отрезка системой случайных отрезков [28].

Функционирование ЛИС, как правило, является временным процессом, который связан с временными изменениями облачных полей над данным пунктом. На больших интервалах этот процесс является сугубо нестационарным, однако в пределах годового хода облачности можно выделить отрезки $\left[t_{0}, t_{s}\right]$ с квазистационарным изменением пропускания в зоне видимости пункта. Особенностью закона распределения количества облачности $j$ (пропускания облачных полей) является его U- или J-образная форма [25-27]. В этих распределениях градации $0 \ldots j_{r}$ и $j_{r}{ }^{\prime} \quad \ldots 10\left(j_{r}=2,3 ; j_{r}{ }^{\prime}=7,8\right)$ имеют наибольший вес. В частности, поля с градациями $0 \quad \ldots 2,8 \ldots 10$ для рассматриваемых в [27] пунктов имеют зимой вероятность повторения 0,9 и 
выше, а поля с градациями 3 ... 7 - около 0,1. Летом соответственно эти вероятности имеют значения $0,75 \ldots 0,9$ и $0,1 \ldots 0,25$. Та же тенденция наблюдается и внутри группировок градаций 0 ... 2 и 8 . 8 . 10 [26,27]. Безоблачные поля $(j=0$ или $Z=1)$ и поля сплошной облачности $(j=10$ или $Z=0)$ для различных климатических районов с градациями $0 \ldots 2$ и $8 \ldots 10$ имеют вес летом: $\quad P_{0} / P_{0-2} \approx P_{10} / P_{8-10} \approx 70 \%$, зимой $P_{0} / P_{0-2} \approx P_{10} / P_{8-10} \approx 80 \%$. Отсюда можно сделать вывод, что поля сплошной облачности и ноля без облачности преобладают в зоне видимости данного пункта.

Если теперь интересоваться временным средним пропускания на отрезке стационарности (пренебрегая суточными и междусуточными колебаниями), т. е. величиной

$$
\bar{Z}_{0}=\frac{1}{t_{f}-t_{0}} \int_{t_{0}}^{t_{f}} Z_{0}(t) d t,
$$

где волнистая черта означает усреднение реализации по времени, то можно заключить, что основной вклад при определении $Z_{0}$ вносят поля с пропусканием, близким к нулю (градации $\left.j_{r}{ }^{\prime} . . .10\right)$ или к единице (градации 0 ... $\left.j_{r}\right)$.

Следовательно, вводя аппроксимацию $\bar{Z}_{0}^{0-j_{\Gamma}}=1$ и $\bar{Z}^{j^{\prime \prime}}=0$, т. е. используя только два класса полей — прозрачные с градациями $0 . . j_{г}$ и непрозрачные с градациями $j_{\Gamma}=\left(j_{\Gamma}+1\right) \ldots 10$ можно получить усредненное по времени пропускание, которое будет незначительно отличаться от определяемого по

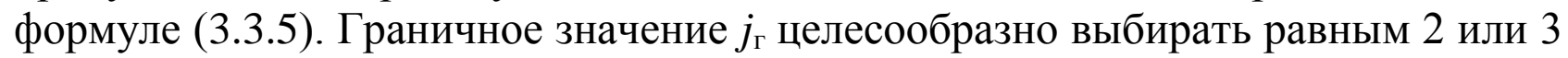
в зависимости от применяемых группировок градаций. Такая аппроксимация является полезной, так как позволяет отказаться от изучения тонкой пространственной структуры полей с дискретными неоднородностями и при определении $\bar{Z}_{0}$ перейти к статистическим временным характеристикам полей с градациями $0 \ldots j_{\Gamma}$ : длительности существования полей $\tau^{0-j_{\Gamma}}$ и средней частоты их появления $\mu=1 / \bar{T}^{0-j_{T}}$, где $\bar{T}^{0-j_{T}}-$ средний период следования прозрачных полей. Тогда при $\left(t_{1}-t_{0}\right) \rightarrow \infty$

$$
\bar{Z}_{0}=\sum_{i=1}^{n} \tau_{i}^{0-j_{\Gamma}} /\left(t_{f}-t_{0}\right)=\mu \tau^{-0-j_{\Gamma}}=p^{0-j_{\Gamma}},
$$

где $\quad \tau^{-0-j_{\Gamma}}=\bar{Z}_{0}=\sum_{i=1}^{n} \tau_{i}^{0-j_{\Gamma}} / n ; \mu=n /\left(t_{f}-t_{0}\right)=1 / \bar{T}_{i}^{0-j_{T}} . \quad$ Последнее $\quad$ соотношение определяет вероятность существования прозрачных полей в зоне видимости в любой момент времени при $\left(t_{f}-t_{0}\right) \rightarrow \infty$ или, другими словами, вероятность проведения сеанса связи в любой момент времени, т. е. $P_{c} \approx \bar{Z}_{0} \approx p^{0-j_{T}}$.

Следовательно, знание временных характеристик $\tau^{0-j_{\Gamma}}$ и $\bar{T}^{0-j_{\Gamma}}$ позволяет легко определять усредненное по времени пропускание (или вероятность оптического кон- такта с ИСЗ) в любой момент времени или в течение некоторого интервала времени, используя теорию совпадения случайных импульсных потоков [29].

Временные параметры облачных полей стали исследоваться сравнительно 
недавно, поскольку для этого должны использоваться наблюдения с небольшой дискретностью. В работах $[6,7]$ по результатам ежечасных наблюдений произведена оценка непрерывной продолжительности существования облачных полей в различных метеорологических пунктах, разнесенных на значительные расстояния. Согласно этим работам функция распределения длительности существования облачных полей градации $j$ аппроксимируется зависимостью вида

$$
P\left(\bar{\tau}^{j} \leq \tau\right)=1-\exp \left[-\beta\left(\tau^{j} / \bar{\tau}^{j}\right)^{\gamma}\right],
$$

где $\beta=\left\lfloor\Gamma(1 / \gamma+1)^{y}\right\} \Gamma(\mathrm{x})$ - гамма-функция, а параметр $\gamma$ зависит от условий формирования в эволюции облачности.

Функции распределения (3.3.6) соответствует закон Вейбулла

$$
W\left(\tau^{j}\right)=\left(\frac{\tau^{j}}{a}\right)^{b-1} \frac{b}{a} \exp \left[-\left(\frac{\tau^{j}}{a}\right)^{b}\right], b=\gamma,
$$

со средней продолжительностью жизни облачного поля $\tau^{j}=a \Gamma(1+1 / b)$.

Параметр $\gamma[6,7]$ слабо зависит от сезона и места расположения пункта, а определяется преимущественно внешним масштабом и морфологическим типом полей. Так, для полей макромасштаба с пропусканием $j=0 ; 10 \gamma=0,73$, а для полей переменной облачности $(j=1 \ldots 9) \quad \gamma=0,89$ [6]. Исследования длительности существования облачных полей с учетом их морфологии показали, что для слоистых облаков $\gamma=0,83$, а для кучевых $\gamma=0,97$ [7], т. е. для полей Кучевой облачности величине $\tau^{j}$ распределена по экспоненциальному закону.

Средняя длительность существования полей соответствующих градаций ${ }_{\tau}^{-j}$, особенно полей макромасштаба существенно зависит от пространственновременных интервалов (координат пункта и времени года). Средняя длительность существования безоблачных полей $\left(\bar{\tau}_{\tau}^{-0}\right.$ и $\left.\stackrel{-10}{\tau}^{-10}\right)$, и полей сплошной облачности, определяемых макроциркуляционными процессами атмосферы, изменяется по территории от 3 до 16 ч и имеет ярко выраженный годовой ход. В то же время величина $\frac{-1-9}{\tau}$ колеблется в течение года по территории страны от 1,3 до 4,5 ч и мало изменяется над данным пунктом (годовая амплитуда не превышает 1,5 ч).

Следовательно, использование среднестатистических временных характеристик облачных полей позволяет легко определять вероятность проведения сеанса измерений ЛИС. 


\section{4 Анализ эффективности применения комплекса лис в условиях облачных полей}

Для анализа потенциальной эффективности комплекса пространственноразнесенных ЛИС целесообразно использовать группу вероятностных показателей, наиболее полно отражающих степень устойчивости комплекса по отношению к мешающему действию облачных полей, и в частности вероятность оптического контакта в любой момент времени или в течение некоторого заданного интервала времени $\Delta \mathrm{t}_{3}$.

Анализ эффективности применения комплекса ЛИС целесообразно проводить на базе следующей частной математической модели. В соответствии с моделью пропускания облачных полей в зоне видимости будем отождествлять движение или любые эволюционные изменения облачных полей над пунктами размещения ЛИС со случайными импульсными потоками $\Omega_{3}$. Длительности существования над пунктом прозрачных полей (с градациями 0

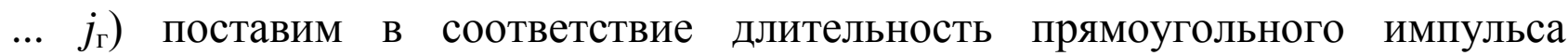
единичной амплитуды, а времени существования непрозрачных полей (с градациями $\left.j^{\prime \prime}=\left(j_{\Gamma}+1\right) \ldots 10\right)$ - длительность паузы потока. Тогда комплекс ЛИС на интервале стационарности можно рассматривать как динамическую систему с дискретными состояниями [29]. Состояния системы различаются по числу пунктов, способных в данный момент времени или в несовпадающие моменты времени $t_{\mathrm{s}}$ осуществлять оптический контакт с исследуемым подвижным объектом с вероятностью, близкой к единице. Другими словами, состояния измерительного комплекса будут характеризоваться процессом совпадения импульсных потоков, отображающих временную изменчивость облачных полей над каждым пунктом и переводящих динамическую систему (комплекс ЛИС) из одного состояния в другое.

Комплекс из $n$ пунктов имеет $k=0,1, \ldots, n$ состояний:

$$
W\left(\tau^{j}\right)=\left(\frac{\tau^{j}}{a}\right)^{b-1} \frac{b}{a} \exp \left[-\left(\frac{\tau^{j}}{a}\right)^{b}\right], b=\gamma,
$$

и может находиться в каждом из них в произвольный момент времени $t \mathrm{c}$ вероятностью [29]

$$
P_{n, k}=\left.\frac{1}{k} \frac{d^{k}}{d \eta^{k}} \coprod_{s=1}^{n}\left(q_{s}^{0-j_{\Gamma}}+\eta p_{s}^{0-j_{\Gamma}}\right)\right|_{\eta=0},
$$

где $q_{s}^{0-j_{r}}=1-p_{s}^{0-j_{\Gamma}}-$ вероятность отсутствия облачных полей с градациями $0 \ldots j_{\Gamma} ; q_{s}^{0-j_{\Gamma}}=\mu \tau_{s}^{-0-j_{\Gamma}}, \sum_{k=0}^{n} p_{n, k}=1$.

Если стратегия измерений предусматривает установление оптического контакта с объектом хотя бы одним из пунктов $(k \neq 0)$, что характерно при локации Луны или стационарных ИСЗ $[12,30]$, то вероятность оптического контакта можно определить на основании соотношения:

$$
P_{O K}=1-p_{n . o}=1-\prod_{s=1}^{n}\left(1-q_{s}^{0-j_{\Gamma}}\right),
$$


где $p_{n, o}$ - вероятность того, что комплекс из $n$ пунктов закрыт облачными полями.

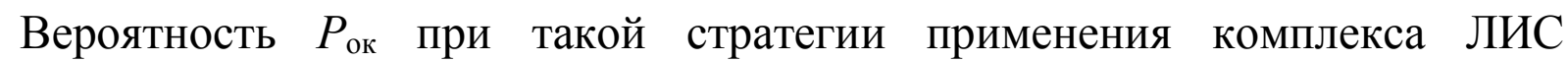
оценивалась [30] для трех статистически слабо зависимых пунктов (рис. 3.5). В работе [30] использовалась вероятность отсутствии облачности на линии визирования $p(\xi)$ для двух углов места $\alpha=\pi / 2-\xi\left(\alpha=30^{\circ}\right.$ и 70 $)$, которая подставлялась и соотношение (3.4.2). вместо вероятности $p_{s}^{0-j_{T}}$. Для каждого из указанных пунктов вероятность $p(\xi)$ изменяется в пределах $0,4 \ldots \ldots, 8$, вероятность же оптического контакта с ИСЗ хотя бы одним из трех пунктов в среднем за год составляет $0,94\left(\xi=60^{\circ}\right)$ и $0,96\left(\xi=20^{\circ}\right)$, при этом ее годовая

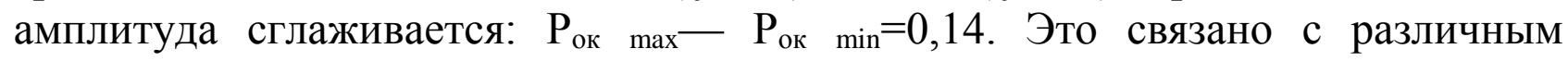
характером годового хода облачности над данными пунктами, о чем свидетельствуют результаты районирования территории Сев. Америки [21, 22].

Если же стратегия применения ЛИС заключается в использовании нескольких пунктов $(r)$ в одновременные или разновременные моменты времени, то вероятность оптического контакта $(k \geq r)$

$$
P_{O K}=1-p_{n, r}=\sum_{k=r}^{n} p_{n, k} \text {. }
$$

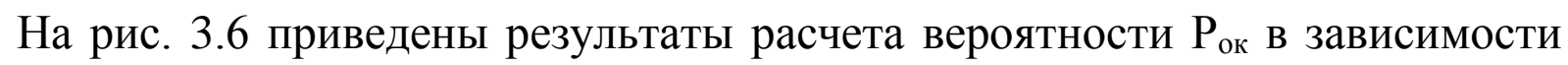
от числа пунктов для двух стратегий применения $k \neq 0$ и $k \geq 3$. При этом использованы условия по облачности, которые приближенно соответствуют условиям рис. 3.5 [30] в наихудший и наилучший периоды. Кривые 1 характеризуют зависимость $P_{\text {ок }}(n)$ при $p_{s}^{0-j_{\Gamma}}=0,7$ (все пункты расположены в районе с благоприятными условиями на некотором временном интервале стационарности), а кривые 3 - при $p^{0-j_{\Gamma}}=0,3$ (район с неблагоприятными условиями на данном временном отрезке). Кривые 2 получены при комбинировании пунктов с антикоррелированными условиями на рассматриваемом временном интервале, т. е. каждую пару составляют пункты с $p_{s}^{0-j_{T}}=0,7$ и $p^{0-j_{T}}=0,3$. Последний вариант размещения является оптимальным,

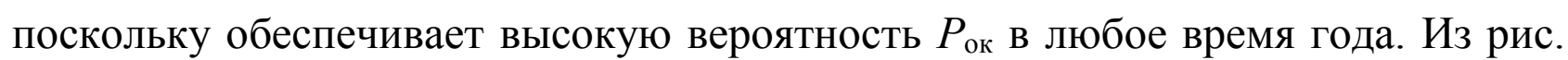
3.6 видно, что задета измерений параметров движения объекта несколькими пунктами я моменты времени $t_{s}$ будет решаться с вероятностью $P_{\text {ок }}=0,9$ при значительно большем числе пунктов.

Если не требуются оперативные измерения (например, при решении

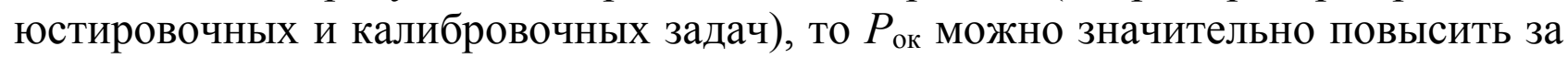
счет временного разнесения сеансов измерений. Вероятность установления оптического контакта в течение некоторого заданного интервала времени для $k \neq 0$ можно определить из следующего соотношения [29]:

$$
P_{\text {ОК }}\left(\Delta t_{3}\right)=1-p_{n, o}+p_{n, o} p_{n, o}^{\prime}\left(\Delta t_{3}\right),
$$

где 


$$
p_{n, o}^{\prime}\left(\Delta t_{3}\right)=\frac{1}{\bar{\tau}_{n, o}} \int_{0}^{\Delta t_{3}}(\tau) d \tau
$$

Здесь $W_{n, o}$ и $\bar{\tau}_{n, o}$ - плотность вероятности и среднее время пребывания системы в нулевом состоянии (все пункты закрыты облачными полями). Если $W_{\mathrm{n}, \mathrm{o}}(\tau)$ подчиняется распределению Вейбулла, то

$$
p_{n, o}^{\prime}\left(\Delta t_{3}\right)=\frac{1}{\tau_{n, o}} \int_{0}^{\Delta t_{3}} \exp \left[-\left(\frac{x}{a}\right)^{b}\right] d x .
$$

При $\Delta t_{3} \rightarrow \infty p_{n, o}^{\prime}\left(\Delta t_{3}\right) \approx 1$. Это условие уже примерно выполняется при $\Delta t_{3}>>\tau_{s}^{-0-j_{\Gamma}}$.

Приближенно вероятность $P_{\text {ок }}\left(\Delta t_{3}\right)$ можно оценить, полагая $b \approx 1$, с помощью соотношения

$$
P_{O K}\left(\Delta t_{3}\right)=1-\prod_{s=1}^{n}\left(1-\mu \tau_{s}^{-0-j_{\Gamma}}\right) \exp \left(-\frac{\Delta t_{3}}{\bar{\tau}_{n, o}}\right)
$$

где

$$
\tau_{n, o}^{\prime}=\left[\sum_{k=1}^{n}\left(1 / \bar{\tau}_{s}^{-"-10}\right)\right]
$$

На рис. 3.7 показана зависимость $P_{\text {ок }}\left(\Delta t_{3}\right)$, рассчитанная с помощью (3.4.3) при пространственно-временной стратегии проведения сеанса измерений $(k \neq 0)$. Следовательно, временное разнесение сеансов связи на интервале в несколько суток позволяет получать $P_{\text {ок}}\left(\Delta t_{3}\right) \approx 1$ даже при использовании одной ЛИС.

Если требуется проведение оперативных измерений, то наиболее полно преимущества ЛИС проявляются при их комплексировании с радиотехническими системами. В этом случае радиотехнические измерения могут быть откалиброваны по лазерным измерениям, полученным в моменты отсутствия облачности на линии визирования. Так, в работе [13] описана наземная система для измерения параметров движения ИСЗ, в которой поиск объекта и предварительное наведение лазерного луча осуществляется с помощью радиолокатора AN/FPQ-6. Точность определения угловых координат ИС3 по радиоканалу составляет около 15". Поэтому угловая расходимость лазерного пучка выбирается более 15" ( 20") для полного перекрытия области неопределенности местоположения объекта. При среднеквадратической погрешности измерения дальности радиолокатора $\sigma_{R}=2$ м может быть сформирован стробирующий импульс длительностью 60 нс, который открывает приемник оптических сигналов и обеспечивает прием 99,99\% отраженных от ИСЗ оптических сигналов.

Совмещенная система не только суммирует положительные стороны 
лазерной и радиотехнической систем, но и обладает к тому же дополнительными преимуществами. Расположение лазерной и радиолокационной систем на общем приводе позволяет использовать для них одни и те же топографические и геодезические данные, а также общую систему синхронизации. При этом динамические режимы работы радио и оптического дальномеров одинаковы. Линия визирования на объект для ЛИС и РЛС проходит через одни и те же участки атмосферы. Поскольку локатор СДЧ диапазона может функционировать при любых погодных условиях, непрерывность потока информации гарантируется даже при сплошной облачности. При переменной облачности показания радиодальномера могут быть откалиброваны с помощью показаний лазерного дальномера по отдельным точкам.

Радиолокатор AN/FPQ-6 может осуществлять сопровождение цели размером до $1 \mathrm{M}^{2}$, находящейся на расстоянии свыше 1000 км. В системе используется 8,8-м параболический отражатель. Излучение на него подается от 4-рупорного моноимпульсного облучателя системы Кассегрена. В первичном отражателе имеется отверстие диаметром 36 см для оптического телескопа, смонтированного за СВЧ отражателем на оси вертикальной наводки. Этот телескоп с фокусным расстоянием 203 см и диаметром 20 см предназначен для слежения за целью. Перед задним выходным люком, за которым устанавливается ТВ камера, может быть установлен дополнительный телескоп. На приемник через дополнительный боковой люк с помощью делителя отводится 30\% энергия, падающей на телескоп. Это позволяет осуществлять прием лазерного излучения, не нарушая обычной, процедуры слежения. Антенный привод, обеспечивающий точность наведения по азимуту и углу места около 10", смонтирован на гидростатических подшипниках и приводится в движение гидравлическим двигателем.

Важным элементом системы радиолокатора является цифровая ЭВМ. Она осуществляет целеуказание с использованием номинальных данных по орбитальному движению ИСЗ в реальном масштабе временя с учетом поправок на высоту установки, преломление в атмосфере и т. п.Лазерная измерительная система состоит из передатчика, приемника, измерителя временных интервалов, устройства синхронизации. Передатчик представляет собой рубиновый лазер с энергией импульса 1 Дж, частотой следования импульсов 1 Гц и с исходной расходимостью пучка 5 мрад [13]. Приняв специальные меры, можно получить длительность импульса 30 нс. Управление лазерной установкой питания осуществляется локаторной системой синхронизации, подающей импульсы с частотой 1 Гц. Поперечный размер лазерного пучка с помощью телескопа и коллимирующих линз доводится примерно до 20 см, а расходимость пучка — до 0,5 мрад. Лазерная головка совместно с юстировочным механизмом и телескопом закрепляются на оси вертикальной наводки радиолокатора непосредственно под его зеркалом.

Описанная система была использована для обнаружения и сопровождения спутника GEOS-II. Оказалось, что результаты измерения дальности с помощью лазера отличаются от данных, полученных с помощью радиолокатора, 
примерно на 8 м, при этом среднеквадратическая погрешность измерений составляет примерно 0,8 м. Полученные результаты следует рассматривать как предварительные, поскольку в первых экспериментах не проводилось оптимизации режима работы системы.

Рассмотренные в этой главе методы повышения эффективности функционирования ЛИС связаны с принципами применения измерительных систем в условиях возмущающих (и прежде всего облачных) полей атмосферы. В значительной степени их дополняют методы, реализуемые на уровне одной ЛИС. Среди них особенно перспективными являются методы адаптации структуры ЛИС к турбулентным искажениям сигнала. 


\section{Глава 4 \\ Характеристики режимов работы оптикоакустических устройств}

\section{1 Режим Рамана-Ната}

Рассмотрим когерентный ОА процессор, устройство которого показано на рис.4.1 [ 1 ]. В процессоре используются первые порядки дифракции. В приближении, что частота ультразвуковой ( УЗ) волны не очень велика, и создаваемая в звукопроводе фазовая решетка одномерна, можно считать, что амплитуда электрического поля световой волны, дифрагировашей в первый максимум, есть $E_{ \pm 1}=J_{ \pm 1}(\Psi)$, где $J_{ \pm 1}$ - функция Бесселя I- го порядка. При малых фазовых возмущениях $\Psi<<1$ имеем $E_{ \pm 1} \approx \pm \Psi$. Если сигнал на входе УЗМС имеет сложную форму, то каждая его спектральная компонента дает свой вклад в дифракционное поле каждого дифракционное поле каждого дифракционного максимума. Пройдя последовательно через два УЗМС I, световая волна будет иметь вид:

$$
\mathrm{E}=\exp \{i[2 \pi v t+\Psi(x, t)]\}
$$

где

$$
\Psi=\Psi_{o}\left[\mathrm{~g}\left(\mathrm{t}-\frac{x}{v}\right) \cos \Omega\left(\mathrm{t}-\frac{x}{v}\right)+\mathrm{r}\left(\mathrm{t}+\frac{x}{v}\right) \cos \Omega\left(t+\frac{x}{v}\right)\right.
$$

$\mathrm{g}(\mathrm{t})$ - анализируемый сигнал, $\mathrm{J}(\mathrm{t})$ - опорный сигнал, $\Psi_{o}$ - постоянная, зависящая от мощности звуковой волны, $\vartheta$ - скорость УЗ волны, Х- координата, $\Omega$ круговая частота. Линза 2 осуществляет фурье - преобразование выходного излучения и позволяет получить в фокальной плоскости пространственное распределение интенсивности - спектр мощности сигнала. С помощью маски 3 экранируется засветка от излучения, распространяющегося в направлении нулевого порядка. Вторая линза 4 осуществляет обратное преобразование фурье и формирует распределение интенсивности, соответствующее выражению:

$$
\mathrm{I}(\mathrm{x}, \mathrm{t})=\left|E_{ \pm 1}\right|^{2}=E_{ \pm 1} E_{ \pm 1} * \approx \Psi \Psi^{*}
$$

то есть

$$
\mathrm{I}(\mathrm{x}, \mathrm{t})=\Psi^{2}\left|g\left(t-\frac{x}{v}\right) \cos \Omega\left(t-\frac{x}{v}\right)+\mathrm{r}\left(\mathrm{t}+\frac{x}{v}\right) \cos \Omega\left(t+\frac{x}{v}\right)\right|^{2}
$$

фотоприемник (ФП) 5 с широкой апертурой выполняет интнгрирование этого распределения интенсивности по координате. В результате на выходе получим сигнал (фототок) с детектора:

$$
\begin{gathered}
i_{\alpha}=\Psi_{0}^{2} \int_{0}^{L}\left\{\frac{1}{2} \mathrm{~g}^{2}\left(\mathrm{t}-\frac{x}{v}\right)\left[1+\cos 2 \Omega\left(t-\frac{x}{v}\right)\right]+\right. \\
+\frac{1}{2} \mathrm{r}^{2}\left(\mathrm{t}+\frac{\mathrm{x}}{\mathrm{v}}\right)\left[1+\cos 2 \pi\left(\mathrm{t}+\frac{\mathrm{x}}{\mathrm{v}}\right)\right] \mathrm{dx}+\psi_{0} \cos 2 \pi \mathrm{t} \int_{0}^{1} \mathrm{~g}\left(\mathrm{t}-\frac{\mathrm{x}}{\mathrm{v}}\right) \mathrm{r}\left(\mathrm{t}+\frac{\mathrm{x}}{\mathrm{v}}\right) \mathrm{dx}
\end{gathered}
$$

Интегрирование в (4.1.5) выполняется в пределах пространственного окна L OA ячейки. Когда длина УЗМС существенно превышает длину УЗ волны, то все 
члены, содержащие сомножителя вида $\cos \left(2 \pi \frac{x}{v}\right)$ при интегрировании дают значение, близкое к нулю. В этом случае имеет:

$$
i_{d}=\frac{1}{2} \psi_{0}^{2} \int_{0}^{1}\left[g^{2}\left(t-\frac{x}{v}\right)+r^{2}\left(t+\frac{x}{v}\right)\right] d x+\psi_{0}^{2} \cos 2 \pi t \int_{0}^{1} g\left(t-\frac{x}{v}\right) r\left(t+\frac{x}{v}\right) d x
$$

Второе слагаемое в (4.1.6) представляет собой функцию свертки сигналов $\mathrm{g}(\mathrm{t})$ и $\mathrm{r}(\mathrm{t})$, наложенную на несущую частоту, равную сумме двух частот У3 модуляторов. Фильтр, находящийся на выходе ФП, пропускает частоты, лежащие в окрестности $2 \pi$. Поэтому он позволяет отделить сигнал свертки от медленно меняющегося первого слагаемого в (4.1.6). Таким образом в когерентном ОА процессоре свет на входе ФП является результатом интерференции лучий, дифрагировавших в первом (сигнальном) и втором (опорном) УЗМС.

Основное преимущество низкочастотного ОА процессора- большое время обработки- может быть реализовано при большой длине звукопровода. Однако конструирование оптической системы для равномерной засветки апертуры более 100 мм представляет серьезные трудности ввиду необходимости иметь высококачественную оптическую поверхность и оптически однородную среду на большой длине звукопровода.

Различные оптические дефекты, наведенные в процессе изготовления звукопровода, дают фоновую, то есть «оптический» шум, связанный с рассеянием непродифрагированного излучения на этих дефектах. Мерой, уменьшающей уровень оптического шума, является отказ от склеек в формирующей оптике (линзы 2 и 4 на рис. 4.1). Кроме того, следует учитывать, что при увеличении акустической мощности возникает дополнительная помеха, связанная с перекачкой света из второго порядка первого УЗМС. Величина этой помехи определяется выражением $\left[J_{1}(\psi) J_{2}(\psi)\right]^{2}$ и носит название «перекрёстного» шума. При оптимальном выбранной величине исследуемого сигнала динамический диапазон ОА процессора ( с учетом указанных выше источников шума) не превышает 35 дБ.

\section{2 Режим Брэгга}

В высокочастотных ОА процессорах используется излучение, дифрагировавшее в первый порядок, а свет в каждом УЗМС распространяется под углом Брэгга к фронту УЗ волны. Звукопровод обычно изготавливается из кристаллического материала, так как в стекле УЗ волна испытывала бы большое затухание. Поскольку высокое оптическое качество в кристаллах на большой апертуре недостижимо, то ОА процессоры в режиме Брэгга имеют времена обработки сигнала не более 10 мкс. Режим детектирования может быть как когерентным, так и некогерентным (рис. 4.2). В некогерентном процессоре свет, непродифрагировавший в первом УЗМС 1 во втором УЗМС не попадает. Фильтрация дифрагированного излучения осуществляется с помощью маски 3 (рис. 4.2б). 
Интенсивность излучения, дифрагированного первым УЗМС, пропорциональна $\left|g\left(t-\frac{x}{v}\right)\right|^{2}$, а интенсивность излучения продифрагированная вторым УЗМ, пропорциональна $\left|g\left(t-\frac{x}{v}\right)\right|^{2} \cdot\left|r\left(t+\frac{x}{v}\right)\right|^{2}$

Фотоприемник интегрирует интенсивность света по координате, его фототок определяется, как:

$$
i_{d} \sim \int_{0}^{L}\left|g\left(t-\frac{x}{v}\right)\right|^{2}\left|r\left(t+\frac{x}{v}\right)\right|^{2} d x
$$

Из (4.2.1) видно, что некогерентный ОА процессор производит свертку квадратов амплитуд сигналов.

Высокочастотные OA процессоры за счет более широкой полосы частот могут обеспечивать примерно такую же величену TAY что и низкочастотные. Однако, для радиолокационных применений необходимы большие времена обработки (до 100 мкс). Параметры некоторых экспериментальных образцов ОА процессоров приводятся в таблице 4.2.1.

Характеристика ОА процессоров

Таблица 4.2.1

\begin{tabular}{|l|l|l|l|l|}
\hline $\begin{array}{l}\text { Несущая } \\
\text { частота } \\
\text { МГц }\end{array}$ & $\begin{array}{l}\text { Полоса } \\
\text { частот, } \\
\text { МГц }\end{array}$ & $\begin{array}{l}\text { Время } \\
\text { обработки } \\
\text { мкс }\end{array}$ & $\begin{array}{l}\text { Динамический } \\
\text { диапазон дБ }\end{array}$ & Материал \\
\hline 1000 & 300 & 2 & - & $\mathrm{L}_{i} \mathrm{NBO}_{3}$ \\
\hline 110 & 50 & 7,5 & 10 & $\mathrm{Pbm}_{0} \mathrm{O}_{4}$ \\
\hline 1560 & 64 & 0,25 & 12 & $\mathrm{Al}_{2} \mathrm{O}_{3}$ \\
\hline 30 & 7 & - & - & \multicolumn{1}{c|}{$T \Phi$} \\
\hline
\end{tabular}

Перспективным для разработок ОА процессороов [2] является парателлурит ( $\mathrm{TeO} 2)$, обладающий очен низкой скоростью распространения звука (616 - мlс ) вдоль напрваления 110, что позволяет на небольшой апертуре светового пучка (длина звукового пути $\leq 20$ мм ) получать времена обработки до 30 мко. Режим Брэгга для УЗМС на $\mathrm{TeO} 2$ реализуется на частотах порядка 40-60 МГц.

Достижения в разработках и исследованиях ОА процессоров , предназначенных для обработки сигналов, достаточно полно отражены в отечественной и зарубежной литературе [3-10].

В последние годы в связи с развитием интегральной оптики наметилось направление исследований ОА модуляторов, работающих в режимые Брэгга на поверхностных акустических волнах (ПАВ ), а также с использованием матричных фотоприемников на выходе устройств [ II-14 ]. В работе [13] описан анализатор, работающий в реальном масштабе времени и использующий OA ячейку из LiNBO3 с ПАВ (рис.4.3 ). Получена 
дифракционная эффективность около $4 \%$ при входной акустической мощности около 100 мВт . Спект шириной 50 МГц (при Vрез=90МГц ) проанализирован с разрешением 300 кГц . Время обработки составило 6,8 мкс . Динамический диапазон 27 дБ . Для регистрации дифрагированного излучения использовался детектор из матрицы фотодиодных структур с переносом заряда (ПЗС матрица). Больший динамический диапазон ; чем в обычном ОА спектроанализаторе, достигается в варианте спектроанализатора интерференционного типа [15], в котором брэгговская ячейка формирует референтную волну с целью получения интерференционной структуры в Фурье - плоскости . (рис. 4.4 ) . В работе [8] описан двумерный ОА спектроанализатор, в котором используется два одномерных ОА спектроанализатора с временным интегрированием и разрешением, равным $\mathrm{N}$ элементам. Обьединение одномерных спектроанализаторов позволяет получить до $N^{2}$ разрешаемых элементов в реальном масштабе времени. Таким образом сформулировано [8] принципиальное положение, а именно : несмотря на то , что ОА устройства , работающие в качестве пространственных модуляторов света, по существу одномерны, тем не менее на их основе возможна обработка двумерных сигналов (в том числе изображений ).

В ряде задач радиотехники (например, при обработке электрических сигналов в реальном масштабе времени) оказывается необходимым проведение спектрального анализа с разрешающей способностью, значительно превышающей возможность существующих спектрометров. С этой целью разработаны различные оптические спектроанализаторы с использованием некогерентного света, как с пространственным, так и с временным интегрированием [15-18], позволяющих осуществлять многоканальный анализ - Спектроанализатор с пространственным интегрированием позволяет реализовать порядка 2000 независимых отсчетов по частоте при разрешающей способности $\approx 20$ дБ.

В спектроанализаторе с временным интегрированием число независимых частотных каналов достигает 1000 , динамический диапазон $\approx$ 26 дБ. Оба типа анализаторов отличаются в основном способом ввода анализируемого сигнала и базисных функций в оптический тракт.

Методы спектральной обработки сигналов базируются на использовании информации, содержащейся как в амплитудном спектре сигналов, так и в комплексном спектре, например, при посстановлении сигналов. Анализатор комплексного спектра с пространственным интегрированием [19] основан на использовании муаровой картины. Так как на перестройку муаровой картины в исследуемом частотном диапазоне требуется определенное время, то анализаторы такого типа не могут быть в не которых случаях использованы для анализатора сигналов в реальном масштабе времени.

Анализатор комплексного спектра с временным интегрированием, позволяющий определять спектры в реальном масштабе времени, содержит ЭЛТ, предназначенную для ввода исследуемого сигнала в оптический 
вычислительный канал, фототранспорт с плотностной записью квадратурных базисных функций, оптическую проекционную систему, фотоприемную матрицу, блок электронных интеграторов, коммутатор и блок функционального преобразования фурье - трансформант.

Луч на экране ЭЛТ, перемещаясь по растру, последовательно пересекает все записи базисных функций и модулируется тем самым в каждый момент времени в соответствии с их коэффициентом пропускания. Яркость свечения луча на экране ЭЛТ изменяется в соответствии с формой исследуемого сигнала, так как этот сигнал после усиления поступает на модуллятор трубки. При этом световые потооки, регистрируемые ячейками фотоматрици, оказываются пропорциональными произведению исследуемого сигнала на базисные функции. После временного интегрирования получаются Фурье - трансформанты, на основании которых в блоке функционального преобразования вычисляются спектральные характеристики сигнала.

В последние годы актуальность приобретают голографические корреляционные методы измерений, и разработка на их основе голографических установок для анализа электрических сигналов в реальном масштабе времени с применением УЗМС[15].С помощью голографического корреляционного метода можно записать Фурье-голограммы анализируемых электрических сигналов и осуществить их объективную классификацию.

$\mathrm{C}$ точки зрения расширения потенциальных возможностей ОА устройств особую важность приобретает разработка оптических процессоров нового класса, получивших название корреляторов с временным интегрированием и позволяющих проводить интегрирование в пределах интервалов длительностью порядка нескольких десятков миллисекунд, которые можно в принципе увеличить за счет последетекторного цифрового интегрирования. В обзорной статье [7] приводится подробной анализ работы такого к коррелятора (рис. 4.5).Представляя коэффициент пропускания СА ячеек непосредственно через возбуждающие их сигналы $V_{1}(\mathrm{t})$ и $V_{2}(\mathrm{t})$ и опуская функцию окна ячейки, можно записать:

$$
\begin{gathered}
t_{1}(x, t)=\exp \left[i V_{1}\left(t-\frac{x}{\vartheta}\right)\right] \rightarrow\left[1+i V_{1}\left(t-\frac{x}{\vartheta}\right)\right] \\
t_{2}(x, t)=\exp \left[i V_{2}\left(t+\frac{x}{\vartheta}-T\right)\right] \rightarrow\left[1+i V_{2}\left(t+\frac{x}{\vartheta}-T\right)\right]
\end{gathered}
$$

где $\mathrm{T}=\frac{L}{\vartheta}$ - время распространения УЗ волны через ОА ячейку,

$$
\begin{aligned}
& V_{1}(\mathrm{t})=B_{1}(\mathrm{t}) \cos \left[2 \pi v_{0} \mathrm{t}+\beta_{1}(\mathrm{t})\right] \\
& V_{2}(\mathrm{t})=B_{2}(\mathrm{t}) \cos \left[2 \pi v_{0} \mathrm{t}+\beta_{2}(\mathrm{t})\right]
\end{aligned}
$$

Знак (+) в (4.2.3) соответствует распространению УЗ волны в обратном направлении во второй ячейке с сигналом $V_{2}(\mathrm{t})$.

После введения “аналитических" для фракций $V_{1}(\mathrm{t})$ и $V_{2}(\mathrm{t})$ в форме:

$$
\widetilde{V_{1}}(\mathrm{t})=B_{1}(\mathrm{t}) \exp \left(2 \pi v_{0} \mathrm{t}\right)
$$




$$
\widetilde{V_{2}}(t)=B_{2}(\mathrm{t}) \exp \left(2 \pi v_{0} \mathrm{t}\right)
$$

Коэффициенты пропускания ОА ячеек принимают вид:

$$
\begin{gathered}
t_{1}\left(\mathrm{x}_{v} t\right)=\left[1+\frac{i}{2} \widetilde{V_{1}}\left(t-\frac{x}{\vartheta}\right)+\frac{i}{2} V_{1}^{*}\left(t-\frac{x}{\vartheta}\right)\right] \\
t_{2}\left(\mathrm{x}_{v} t\right)=\left[1+\frac{i}{2} \widetilde{V_{2}}\left(t+\frac{x}{\vartheta}-T\right) \frac{i}{2} V_{2}^{*}\left(t+\frac{x}{\vartheta}-T\right)\right]
\end{gathered}
$$

Взаимная корреляционная функция сигналов $V_{1}(\mathrm{t})$ и $V_{2}(\mathrm{t})$ определяется выражением:

$$
R_{12}(\tau)=\int V_{1}(\mathrm{t}-\tau) V_{2}(t) d t
$$

На практике обе $\mathrm{OA}$ ячейки (рис.4.5) работают в брэгговском режиме,причем первая из них должна быть повернута на угол Брэгга по часовой стрелке,а вторая - на тот же угол , но против часовой стрелки.При соблюдении этого условия комплексно сопряженные члены в формулах (4.2.8) и (4.2.9) пропадают, и окончательное выражение для коэффициента пропускания приобретает вид:

$$
t_{p e s}(\mathrm{x} t)=\left[1+\frac{i}{2} \widetilde{V_{1}}\left(t-\frac{x}{\vartheta}\right)\right]\left[1+\frac{i}{2} \widetilde{V_{2}}\left(t+\frac{x}{\vartheta}-T\right)\right]
$$

При точной установке ОА ячеек в коллимированном световом пучке через пространственный режекторный фильтр (диафрагму,маску) проходят лишь дифракционные компоненты, соответствующие членам $\frac{i}{2} \widetilde{V_{1}}\left(t-\frac{x}{\vartheta}\right)$ и $\frac{i}{2} \widetilde{V_{2}}\left(t+\frac{x}{\vartheta}-T\right)$. Распределение интенсивности света в плоскости фотодетектора определяется тогда, как:

$$
\begin{aligned}
I_{d}(\mathrm{x} t)=\left|\frac{i}{2} \widetilde{V_{1}}\left(t-\frac{x}{\vartheta}\right)+\frac{i}{2} \widetilde{V_{2}}\left(t+\frac{x}{\vartheta}-T\right)\right|^{2}= \\
=\frac{1}{4}\left|\widetilde{V_{2}}\left(t-\frac{x}{\vartheta}\right)\right|^{2}+\frac{1}{4}\left|\widetilde{V_{2}}\left(t+\frac{x}{\vartheta}-T\right)\right|^{2}+ \\
\quad+\frac{1}{2} \operatorname{Re}\left[V_{1}^{*}\left(t-\frac{x}{\vartheta}\right) \widetilde{V_{2}}\left(t+\frac{x}{\vartheta}-T\right)\right]
\end{aligned}
$$

Расположенный в выходной плоскости матричный фотодетектор осуществляет временное интегрирование сигнала путем накопления снимаемого заряда, величина пропорциональна интенсивности падающего света. Если через $W_{\Delta T}$ обозначить световую энергию, поступающую в точку $x$ матричного детектора за время $\Delta T$, то выходной сигнал интегрирующего детектора в точке $x$ будет пропорционален

$$
\begin{gathered}
W_{\Delta T}(x)=\frac{1}{4} \int_{(\Delta T)} V_{1}^{2}\left(t-\frac{x}{\vartheta}\right) d t+\frac{1}{4} \int_{(\Delta T)} V_{2}^{2}\left(t+\frac{x}{\vartheta}-T\right) d t+ \\
+\frac{1}{2} \operatorname{Re}\left\{\int_{(\Delta T)} V_{1}^{*}\left(t-\frac{x}{\vartheta}\right) \widetilde{V_{2}}\left(t+\frac{x}{\vartheta}-T\right) d t\right\}
\end{gathered}
$$


Если пределы интегрирования полодить бесконечными, то два первых члена в (4.2.13) обращаются в постоянные, а третий член соответствует искомой корреляционной функции. Таким образом имеем:

$$
W_{\Delta T}(x)=\text { const }+R_{12}\left(\frac{2 X}{V}-T\right)
$$

Матричные фотодетекторы на ПЭС имеют время интегрирования порядка десятков миллисекунд.

Размер пространственного окна ()А ячейки в рассматриваемой схеме (рис.4.5) неограничивает длительности сигнала, но огранчивает пределы изменения аргумента $\mathrm{L}$ корреляционной функции $R_{12}(L)$ интервалом $0 \leq L \leq \frac{L}{V}$. Очевидно, основным недостатком 0А корреляторов с временным интегрированием является наличие в выходном сигнале постоянных составляющих, которые сопровождают искомую корреляционную функцию и вызывают насыщение интегрирующих фотодетекторов. Поскольку они связаны с исходным сигналом как функции времени и пространственных координат, их трудно скомпенсировать при последетекторной обработке сигнала. Тем не менее, несмотря на наличие таких составляющих, методы обработке сигналов, основанные на временном интегрировании сигнала, дают значительный выйгрыш при выполнении согласованной фильтрации. 


\section{Глава 5. Анализ оптико акустических устройств обработки сигналов 5.1. Оптико акустические гетеродинные устройства}

Многообразие сложных сигналов, находящих все более широкое применение в радиотехнических системах, требуют создания универсальных устройств анализа.

Оптикоакустические устройства демодуляциичастотно - модулированных (ЧМ) сигналов [21, 22], работающие в режиме дифракции Брэгга, позволяют выделять в реальном масштабе времени законы изменения ЧМ сигналов:

$$
S(t)=\cos \left\{2 \pi\left[V_{0} t+Y(t)\right]\right\}
$$

Где $V_{0}-$ несущая частота, $2 П Y(\mathrm{t})$ - медленно меняющаяся часть фазы. При этом центр $£(t)$ светового пятна, соответсвующего первому порядку дифракции, перемещается вфокальной плокости фурье - линзы по закону:

$$
\frac{\mathrm{a}}{\ni}(t)=\frac{\tau}{2 v} V_{0} F+\frac{\tau}{v} F Y^{\prime}\left(t-\frac{L}{2 v}\right)
$$

где L- размер окна 0A ячейки, v-скорость УЗ волны F-фокусное расстояние Фурье - линзы. Амплитудно-частотная характеристика (АЧХ) демодулятсра имеет вид:

$$
\mathrm{V}(\mathrm{v})=\operatorname{Si}(2 \pi \beta)-\operatorname{Si}[2 \pi(\beta-4)]+\frac{\operatorname{Sin}^{2(\pi \beta)}}{\pi \beta\left(\frac{\beta}{4}-1\right)}
$$

где $\operatorname{Si}(\mathrm{x})-$ интегральный синус, $\beta=\frac{L}{v}\left(v-v_{0}\right)$. График АЧХ согласно расчету алгоритма (5.3) на ЭВМ имеет линейную зависимость $u(v)$ и v'в области $\left(v^{\prime}\right)<0,25$, где $V^{\prime}=\frac{L}{4 v}\left(v-v_{0}\right)$ - относительная частота, $U(t)$ выходное напряжение демодулятора.Полоса частот $\mathcal{W}$ демодуляиии равно $1,6 \frac{v}{L^{*}}$ Если изменение частоты $\hat{Y}$ ' (t) ЧМ сигнала $S(t)$ удовлетворяет неравенству то выходное напряжение описывается выражением:

$$
\mathcal{U}(\mathrm{t})=\mathrm{G} \Psi^{\prime}\left(\mathrm{t}-\frac{L}{2 v}\right)
$$

где G-крутизна линейного участка АЧХ.

В оптической обработке информации особое место занимают оптические гетеродинные корреляторы (ОГК), основанные на использовании голограм много пространственно- временного модулятора в фуье-плоскости когерентнооптической системы для совмещения в пространстве двух разнесенных когерентных изображений- эталонного и сигнального. При корреляционной обработке неоптических полей (сигналов) в качестве таАких модуляторов могут быть использованы ОА модуляторы, возбуждаемые ЛЧМ сигналами. В процессах обработки сигналов радиолокационных станций(РЛС) и систем радиосвязи необходимо определять либо огибающую корреляционной функции сигналов, либо полностью воспроизводить корреляционную функцию с высокочастотным заполнением. Подобные операции способны осуществлать оптикоакустичекие гетеродинные корреляторы (ОАГК), иногда называемые для кратности гетеродинными процессорами. 
В ОАГК “несущая” ,которая переносит информацию, формируется в результате гетеродинного смещения на фотодетекторе двух оптических сигналов с разделенными частотными полосами.

В системе, схема которой приведена на рис.5.I, пространственный фильтр пропускает дифракционные компоненты нулевого и первого порядков, а точечная диафрагма перед фотодетектором обеспечивает пропускание только дифракционной компоненты $+\mathrm{I}$-го порядка.Роль фототранспартанта $\boldsymbol{t}_{\boldsymbol{m}}$ на рис.5.I может играть вторая ОА ячейка с амплитудой функцией пропускания вида:

$$
t_{m}(x, t)=1+i \widetilde{S_{2}}(\mathrm{x}+\vartheta t)+i \widetilde{S_{2}^{*}}(\mathrm{x}+\vartheta t)
$$

Амплитуда волны на выходе системы описывается выражением [7] :

$$
\mathrm{U}(\mathrm{x}, \mathrm{t})=\left\{\left[1+\frac{1}{2} \widetilde{S_{1}^{*}}(x-\vartheta t)\right]\left[1+\frac{1}{2} \widetilde{S_{2}}(x)+\frac{1}{2} \widetilde{S_{2}^{*}}(x)\right]\right\} \times \operatorname{rect}\left(\frac{x}{L}\right)
$$

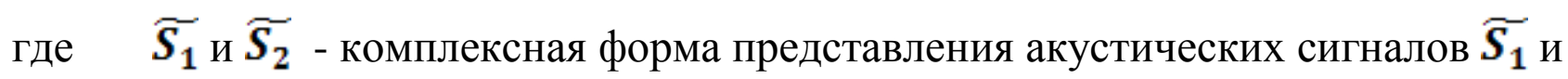
$\widetilde{S_{2}}$, связанных с сигналами $V_{1}$ и $V_{2}$ на входе ОА ячеек в форме

$$
\boldsymbol{S}(\boldsymbol{x}-\boldsymbol{v} \boldsymbol{t})=\boldsymbol{m} \boldsymbol{V}(\boldsymbol{t}),
$$

где $\boldsymbol{m}$ - коэффициент пропорциональности.

$\boldsymbol{S}(\boldsymbol{x}-\boldsymbol{\vartheta} \boldsymbol{t})$-сигнал, перемещающийся относительно окна;

$\boldsymbol{S}_{2}(\boldsymbol{x})$-стационарный сигнал, характеризующий транспарант,

$\boldsymbol{S}_{2}(\boldsymbol{x}-\boldsymbol{\vartheta} \boldsymbol{t})$-акустооптический сигнал, движущийся относительно окна, $\boldsymbol{r e c t}\left(\frac{\boldsymbol{x}}{\boldsymbol{L}}\right)$ означает единичную прямоугольную функцию.

Чтобы пренебречь влиянием окна, ширина сигнала $\boldsymbol{S}_{1}(\boldsymbol{x})$ не должна превышать ширины окна L . Это условие означает, что длительность фильтруемого сигнала не должна превышать времени $\frac{\boldsymbol{L}}{\boldsymbol{v}}$ прохождения акустической волны через ОА ячейку $\frac{L}{\vartheta} \leq \mathbf{4 0}$ мкс.

В выражении (5.6) члены, описывающие дифракционные компоненты первого порядка $\frac{1}{2} \widetilde{S_{1}^{*}}(x-\vartheta t)$ и $\frac{1}{2} \widetilde{S_{2}^{*}}(x)$, содержат множитель пространственной несущей вида $\exp \left(-\boldsymbol{i} 2 \pi f_{0} x\right)$, где $\boldsymbol{f}_{0}-$ пространственная частота, и соответствуют волнам, проходящим к фотодетектора достаточно органичиться рассмотрением волнового поля вида:

$$
U_{d}(x, t)=\left\{\frac{1}{2} \widetilde{S_{1}^{*}}(x-\vartheta t)+\frac{1}{2} \widetilde{S_{2}^{*}}(x)\right\} \operatorname{rect}\left(\frac{x}{L}\right)
$$

Для интенсивности (фототока детектора) имеем:

$$
\boldsymbol{i}_{d}(\boldsymbol{t})=\int_{0}^{L}\left|\boldsymbol{U}_{d}(\boldsymbol{x}, \boldsymbol{t})\right|^{2} d x
$$

Распределение интенсивности (5.8) содержит член $\boldsymbol{S}_{1}(\boldsymbol{x}-\boldsymbol{\vartheta} \boldsymbol{t})$ связанный с сигналом . Искомая взаимная корреляционная функция описывается : 


$$
R_{12}(\vartheta t)=\operatorname{Re}\left\{\int_{0}^{L} \widetilde{S_{1}^{*}}(x-\vartheta t) \widetilde{S_{2}^{*}}(x) \operatorname{rect}\left(\frac{x}{L}\right) \mathrm{dx}\right.
$$

Если сигнал фотодетектора пропустить через полосовой фильтр с несущей частотой $\boldsymbol{v}_{\mathbf{0}}=\boldsymbol{\vartheta} \boldsymbol{f}_{\mathbf{0}}$, то можно получить на выходе электрический сигнал, пропорциональный функции $\boldsymbol{R}_{\mathbf{1 2}}(\boldsymbol{\vartheta} \boldsymbol{t})$.

Возьмем вариант ОАГК с подавленной дифракционной компонентой нулевого порядка и прохождением компонент + I-го и $-\mathrm{I}-г о$ порядков (центры частотных полос этих фомпонент смещены на $\pm v_{0}$ ). В этом случае выходной сигнал фотодетектора, регистрирующего интенсивность света в пределах точечной диафрагмы, имеет вид:

$$
i_{\alpha}(t)=\left|R_{12}(V t)\right|^{2}=1 / 2 r_{12}^{2}(V t)+1 / 2 r_{12}^{2}(V t) \cos \left[2 \pi\left(2 v_{0}\right) t+2 \Theta_{12}(V t)\right]
$$

где $\mathrm{r}_{12}$ и $\Theta_{12}$ - амплитудная и фазовая части функции

$$
\mathrm{R}_{12}(\mathrm{Vt})=1 / 2 \mathrm{r}_{12}(\mathrm{Vt}) \cos \left[2 \pi \mathrm{f}_{0} \mathrm{Vt}+\Theta_{12}(\mathrm{Vt})\right]
$$

Для выделения второго члена в (3.10), имеющего высокочастотную несущую $2 v_{0}$, требуется полосовая фильтрация. Для получения на выходе значений $\mathrm{r}_{12}(\mathrm{Vt})$ и $\Theta_{12}(\mathrm{Vt})$ требуется дополнительная обработка сигнала. Возможна реализация ОАГК с компактной конфигурацией, когда ОА ячейка транспарант (или две ОА ячейки с обращенным входом) располагаются в непосредственной близости друг от друга (рис.5.2). При этом их действие можно описать единой функцией пропускания, равной произведению функций пропускания ОА ячейки и транспаранта:

$$
\begin{aligned}
t_{\text {pes }}(x, t)=t(x, t) t_{m}(x)=\{1+1 / 2 & \exp (i \Theta) \hat{S}_{1}(x-V t)+1 / 2 \exp (i \Theta) \hat{S}_{1}{ }^{*}(x-V t)\left[1+1 / 2 \hat{S}_{2}(x)\right. \\
& \left.\left.+1 / 2 \hat{S}_{2}{ }^{*}(x)\right]\right\} \operatorname{rect}(x / L)
\end{aligned}
$$

Фазовый множитель $\exp (\boldsymbol{i} \boldsymbol{\theta})$, зависящий от расстояния между ОА ячейкой и транспарантом, с целью упращения анализ выбирается равным (-i ).

При расчете светового сигнала в плоскости фотодетектора учитываются в (5.12) лишь члены, содержащие пространственную

несущую вида $\exp \left(\mathbf{i} 2 \pi f_{0} x\right)$, а также члены $\frac{1}{2} \widetilde{S_{1}}(x-\vartheta t)$ и $\frac{1}{2} \widetilde{S_{2}}(x)$. Тогда сигнал на выходе равен:

$$
\mathbf{i}_{\alpha(t)}=\int_{0}^{L}\left|\frac{1}{2} \widetilde{S_{1}}(x-\vartheta t)+\frac{1}{2} \widetilde{S_{2}}(x)\right| 2 \operatorname{rect}\left(\frac{x}{L}\right) \mathrm{dx}
$$

Выражение (5.13) можнопреобразовать к виду (5.9) .

Трудность реализации ОАГК по схеме рис. 5.2 состоит в том, что форма волнового сигнала, прошелшего через ОА ячейку, не должна испытывать существенного изменения на участке распространения между ячейкой и транспарантом, то есть набегом фазы $\exp (i \boldsymbol{\theta})$ в (5.12) можно было бы пренебрегать. Это условие налагает ограничения на величину расстояния между ячейкой и транспарантом и на ширину полосы частот модуляции [23].

Гетеродинная обработка сигнала в плоскости преобразования фурье может быть иллюстрирована типом ОАГК с пространственным 
интегрированием. На рис. 5.3 приводится схема гетеродинного процессора [7], в котором фотодетектор размещается в плоскости преобразования Фурье ОА ячейки после режекторного фильтра, который пропускает только идфракционную компоненту (-I)-го порядка. На фотодетектор приходят два волновых сигнала, один из которых является результатом пространственного преобразования Фурье сигнала

$$
\widetilde{S_{1}}(x-\vartheta t),
$$

a другой - (сигнал местного гетеродина) определяется особенностями оптической системы второго плеча. Обозначим через $\xi$ координату положения плоскости преобразования Фурье, а через $\frac{1}{2} \widetilde{S_{2}{ }^{*}}(-\xi)$ - сигнал гетеродина (причины введения такого вида сигнала будут ясны из дальнейшего). Тогда амплитуду волны, приходящей на фотодетектор, можно представить, как:

$$
\mathrm{Ud}(\xi, \mathrm{t})=\frac{1}{2} \widetilde{S_{1}}(\xi) \exp (-i 2 \pi \xi \mathrm{Vt})+\frac{1}{2} \widetilde{S_{2}^{*}}(-\xi)
$$

где $\widetilde{\boldsymbol{S}_{\mathbf{1}}}(\xi) \exp (-i 2 \pi \xi \mathrm{Vt}) \quad$ - пространственное преобразование Фурье сигнала $\widetilde{\boldsymbol{S}_{\mathbf{1}}}(\boldsymbol{x}-\boldsymbol{\vartheta} \boldsymbol{t})$. Выходной сигнал фотодетектора определяется выражением:

$$
i d(t)=\int_{0}^{L}|U d(\xi, t)|^{2} d \xi
$$

которое в соответствии с теоремой Рэлея можно записать а виде:

$$
\begin{gathered}
i d(t)=\int_{0}^{L}\left|\frac{1}{2} \widetilde{S_{1}}(x-\vartheta t)+\frac{1}{2} \widetilde{S_{2}{ }^{*}}(x)\right| 2 d x= \\
=\frac{1}{4} \int_{0}^{L}\left|\widetilde{S_{1}}(x-\vartheta t)\right| 2 d x+\frac{1}{4} \int_{0}^{L}\left|\widetilde{S_{2}{ }^{*}}(x)\right| 2 d x+\frac{1}{2} \operatorname{Re}\left\{\int_{0}^{L} \widetilde{S_{1}}(x-\vartheta t) \frac{1}{2} \widetilde{S_{2}}(x) d x\right\}
\end{gathered}
$$

где $\widetilde{\boldsymbol{S}_{2}}(\boldsymbol{x})$ представляет собой результат обратного преобразования Фурье распределения $\widetilde{\boldsymbol{S}_{2}}(\xi)$ и является аналитическим сигналом, связанным с действительным сигналом $\boldsymbol{S}_{2}(\boldsymbol{x})$. Первый член выражения (5.16) соответствует низкочастотной составляющей, а второй - сигналу нулевой частоты. Третий (высокочастотный) член имеет форму свертки:

$$
i_{c}(t)=\int_{o}^{L} S_{1}(x-\vartheta t) S_{2}(x) \mathrm{dx}
$$

Выражение (5.17) представляет собой результат фильтрация входного сигнала $V_{1}(t)$ фильтром с импульсной функцией $S_{2}(x)$. При подаче на вход ОА ячейка сигнала $\mathrm{V}(\mathrm{t})=\cos 2 \pi V_{s} t$ в плоскости фотодетектора в точке $\xi=\xi_{s}$ 
возникает световое пятно, частота которого ниже частоты исходного сигнала на величину $V_{s}$. Выходной сигнал фотодетектора (в форме «биений») формируется в результате смешения указанного светового сигнала в точке $\xi=\xi_{s}$ с сигналом $\widetilde{S_{2}^{*}}(-\xi)$. "Биения" имеют форму синусоидального сигнала с частотой $V_{s}$, амплитуда и фаза которого определяются амплитудой и фазой сигнала гетеродина в точке $\xi_{s}$. В работе [15] описан ОА процессор на основе двухплечевой схемы, подробно описанной выше, с той разницей, что в плечо оптического гетеродина поставлена брэгговская ячейка для формирования опорной волны и выработки интерференционной частоты (сигнала "биений") в результате смешения с сигнальным пучком в плоскости линейки фотодетекторов на ПЗС.

Для корреляционной обработки и спектрального анализа радиосигналов применяются также ОА гетеродинные устройства на основе УЗМС с выделением разностной частоты между дифрагированным на УЗ волне и опорным (гетеродинным) световыми пучками. При этом используются схемы с двумя независимыми лазерными источниками $[3,24]$ или вспомогательная решетка [25], или разделение исходного светового пучка на два канала [26].

Возможно построение ОАГК с использованием поляризационного кристаллооптического расщепителя, создающего эффект поперечного сдвига с наложением отдельных дифракционных максимумов.

\section{2. Магнитоакустооптический дефлектор}

Излагаемый ниже вопрос имеет отношение к разработке нового класса оптикоакустических устройств с магнитной связью. Речь идет о расширении функциональных возможностей ОА дефлектора путем осуществления в магнитном поле допольнительного углов брэгговской дифракции при сохранении высокой разрешающей способности дефлектора.

Угол отклонения дифрагированного луча в созданныхна сегодняшный день дефлекторах не превышает 30 угл.минут. Такой результат достигнут [27] при отклонении луча инжекционного лазера $(\lambda=0.91$ мкм) в первом порядке дифракции Брэгга на продольных УЗ волнах в $P B M_{0} O_{4}$ в полосе частот $100 \div 140$ МГц.

В работах [28-31] впервые была реально продемонстрирована возможность увеличения примерно в десять раз угол брзгговской дифракции в дефлекторе, который содержит УЗ ячейку и из магнитоактивного материала: железо - иттриевого гранита (ЖИГ-кристалл) с пьезопреобразователем из $\mathrm{LiNBO}_{3} \cdot$ Когда вдоль стержня ЖИГ прикладывалось внешнее магнитное поле $H$, наблюдался сдвиг брэгговского угла дифракции $\theta_{B}$ лазерного луча $(\lambda=1.15$ мкм) на величину $\theta_{B}=3^{\circ}$ в поле $\mathrm{H}=600 \ni$ на частоте УЗ волны 1,1 ГГц. Столь большой угол отклонения объясняется магнитоакустооптическим эффектом, который состоит в том, что при определенной величине магнитного поля в области сильного магнитокпорного взаимодействия лазерный луч испытывает дифракцию на магнитоупругих волнах, возникающих в магнитоактивной среде УЗ ячейки. 
Предлагаемое в работе [31] устройство - магнмтоакустооптический (MAO) дефлектор (рис.5.4) состоит из лазера I, коллиматора 2, содергащего микрообъектив, диафрагму и обьектив, УЗ ячейки, состоящей из звукопровода 3 и пьезопреобразователя 4, соединенных с генератором пернменяого электрического поля 5, цилиндрической линзы 6 , фотоприемной системы, состоящей из фотоприемника 7 с входной целью и осциллографа 8 , устройства возбуждения магнитного поля, состоящего из соленоида (или системы катушек Гельмгольца) 9 и звукового генератора 10, причем в устройство возбуждения помещена УЗ ячейка, МАО дефлектор работает следующим образом.

Анализируемый электрический сигнал, подаваемый от генератора 5 на пьезопреобразователь 4, возбуждает в звукопроводе 3 бегущую У3 волну, на которой происходит брэгговская дифракция светового пучка, идущего от лазерного источника I и расширенного с помощью коллиматора 2. Дифрагированный в первом порядке луч фокусируется цилиндрической линзой 6 на фотоприемник 7 и регистрируется на осциллографе 8. При наложении на У3 ячейку магнитного поля $H$ в магнитоактивном веществе звукопровода 3 возникают магнитоупругие волны, на которых лазерный луч претерпевает значительную дифракцию. При соответствующей величине внешнего магнитного поля $\mathrm{H}$ в звукопроводе создается область сильного магнитоупругого взаимодействия, отвечающая аномально большому углу брэгговской дифракции $\theta_{B}$.

Для пояснения физического принципа, на котором основана работа МАО дефлектора, рассмотрим брэгговскую дифракцию лазерного излучения на магнитоупругих волнах в ЖИГ- кристалле.Теория магнитоупругих волн применительно к ЖИГ - кристаллу рассмотрена [32]. Для поперечных магнитоупругих волн, распространяющихся вдоль направления < 001> , дисперсионные кривые показаны на рис. 5.5.

Сильную связь со спиновыми волнами обнаруживает только циркулярно поляризованная компонента сдвиговой волны, вращающаяся в направлении свободной процессии, спина . Выражение для волнового числа

$$
\mathrm{K}^{2}=\frac{\omega-\gamma H i+\Delta \omega}{2 \gamma Д}\left\{1 \pm\left[-4 \gamma Д\left(\frac{\omega}{c_{t}}\right)^{2} \frac{\omega-\gamma H_{i}}{\left(\omega-\gamma H_{i}+\Delta \omega\right)^{2}}\right] 1 / 2\right\}
$$

где

$$
\Delta \omega \equiv \gamma\left(Д \frac{\omega^{2}}{C_{t}^{2}}+\frac{B^{2}}{\alpha M}\right)
$$

$\omega$-угловая частота, -постоянная магнитоупругой связи, $\alpha$-упругая постоянная, $D$-обменная постоянная, Ct-скорость поперечной волны в упругом пределе, $\gamma$ гиромагнитное отношение, М-намагниченность насыщения, $\mathrm{H}_{\mathrm{i}}$ внутреннее поле. Знаки (+) и (-) в формуле (5.2.1) обозначают нижнюю и верхнюю ветви дисперсионной кривой соответственно. Если внутреннее поле $\mathrm{H}_{\mathrm{i}}$ принимает значения, близкие к $H_{i}=\frac{1}{\gamma}\left(\omega_{c}-D \frac{\omega_{c}^{2}}{c_{t}^{2}}\right.$, где $\omega_{c}$-частота в точке перекрытия, то 
есть в точке пересечения дисперсионных кривых несвязанных спиновой и упругой волн, то происходит сильное магнитоупругое взаимодействие, что в свою очередь приводит к изменению значения числа $/ \bar{K} /$ и, следовательно, к изменению угла брэгговской дифракции, определяемому из условия:

$$
\sin \theta_{B}=\frac{K}{2 K_{0}} ; \quad K_{0} \equiv \frac{2 \pi}{\lambda}
$$

В работе [3I] описан эксперимент по дифракции лазерного излучения ( $\lambda$ 1,15мкм) на магнитоупругих волнах с частотой 1,1 ГТц в ЖИГ-кристалле. Максимальное изменение угла Брэгга $\Delta \theta_{\mathrm{B}}$, наблюдавшееся при внешнем магнитном поле $\vec{H}$ порядка $600 \exists$, составило $3^{0}$ при изменении поля вдоль стеркня на величину около $20 \ni$ (рис.5.6). 


\section{ЗАКЛЮЧЕНИЕ}

Разработка импульсных источников электромагнитного излучения не ограничивается разумеется одной облостью их применения а именно в волоконно-оптических линиях связи. Можно назвать другие не менее важные проблемы использования импульсных источников в УФ видимой и ИК облостях спекра для оптикоэлектронных устройств обработки распределения и передачи информаций. Так в системах оптической локаций удаленных объектов требуются мощные импульсные лазеры на рубине неодимовом стекле а в оптических вычислительных системах в медицинской аппаратуре в малогабаритных голографических установкахжелательно иметь компактные полупроводниковые лазеры типа ПГЛ полосковой геометрий.

Разработка различных способов формирования световых импульсов переменной длительности с регулируемой тактовой частотой имеет важное значение и для решения вопросов импульснокодовой оптической связи. При этом встает задача расшифровки получаемой информаций посредством анализа фурье- спектра приходящих сигналов. В настоящее время уделяется внимание составлению программ для расчетов на ЭВМ спектров модулированных когерентных и некогорентных последовательностей прямоугольных импульсов и импульсов заданной формы для оптический линий связи. (36-38).

При всем многообразии ОА устройств обработки сигналов предпочтение следует отдать ОА корреляторам с временным интегрированием, использующим в качестве выходных детекторов с накоплением ФД структуры (ПЗС матрицы). Это позволяет в дальнейшем при сопряжении коррелятора с ЭВМ путем цифровой обработки анализировать радиосигналы длительностью в несколько миллисекунд и более. Что касается разрешающей способности современных ОА спектроанализаторов, то она может быть доведена, судя по зарубежным источникам информации [8.c.103], до нескольких кГц и менее с помощью алгоритма ЛЧМ - преобразования. Приведенные в [31] данные указывают на принципиальную возможность создания нового типа ОА приборов на основе магнитоакустооптического взаимодействия, в которых отклонение лазерного луча осуществлялось бы комбинированно: электрическим и магнитным полями. По сравнению с существующими ОА дефлекторами МАО дефлектор обладает следующими преимуществами:

1.MАО дефлектор расширяет функциональные возможности ОА дефлектора вследствие, во-первых, осуществления дополнительного сканирования лазерным лучом меняющимся во времени магнитным полем и, во-вторых, за счет увеличения динамического диапазона углов дифракции $\left(3^{0}\right)$.

2. В технических приложениях МАО дефлектор может оказаться перспективным при работе ОА дефлектора на ПАВ, где угол дифракции мал, в сочетании с полупроводниковыми гетеролазерами, обладающими широкой диаграммой направленности. 


\section{Список литературы}

1. Криксунов Л.З. Системы информаций с ОКГ. Киев, «Техника» 1970,170-174

2. Wigmore K. Grischkowky D. Временное сжатие импульсов света

3. IEEE J. Qant. Elect. 1978

4. Крюков П.Г., Матвеец ,Никогосян Д.Н. перестриаваемый по частоте двухканальный генератор ультракоротких импульсов света. «Квантовая электроника»,1978,т.5,№11,с.2348

5. Каталог на импульсные лазеры на красителях. США,1978.

6. Формирователь лазерного импульсного сигнала. Франц.заявка,КЛ.02 1/26 Н

7. Эйнолф. Генератор наносекундных импульсов для пьезоэлектрического преобразователя. «Приборы для научных исследований», 1971,№3

8. Вейц, Левинсон, Кобас. Метод получения прямоугольных импульсов света высокой интенсивности с временем нарастания порядка микросекунд. «Приборы для научных исследований»,1971,№2,с.75

9. Генерация субнаносекундных оптических импульсов переменной длительности. «ТИЭР», 1969, т.57, с.75

10. Ванюков М.П., Мак А.А., Муратов В.Р. Установка для фотоэл- ктрический регистрация временных спектров коротких световых вспышек. «ОМП»,1958,№2,c.36

11. Минаев В.П., Обод Ю.А., Турков Ю.Г. Импульсный гелийнеоновый лазер для голографических запоминающих устройств. Первая всесоюзная конф.«Проблемы управления параметрами лазерного излучения»,Тезисы докладов ,часть II, Ташкент,1978,c.141

12. Заславская В.Р., Попов Ю.В., Степаненко Н.Ф.,Чижиков Г.Г. Модуляция добротности непрерывного лазера на алюмоиттриевом гранате с целью его применения в импульсной системе оптического гетеродинирования. Первая всесо.конф. Тезисы докладов,Ташкент,1978,с.149

13. Венцель. Определение характеристик импульсных лазеров. «ЗРЭ»,1965,№10,c.75

14. В сб. «Квантовая электроника»,1971,№6,с.97,105,106,120

15. Миронов Ю.М.,Морозов В.Н.,Семенов А,С., Сергеев А.Б. Использование внешней оптической обратной связи для увеличения скорости прямой кодово-импульсной модуляции инжекционного полупроводникового лазера. Первая всесоюзная конф. «Проблемы управления параметрами лазерного излучения» Тезисы докладов,часть II, Ташкент ,1978,c.119-123

16. Арсеньев В.В.,Матвеев И.Н., Степанов А.Н. Управление параметрами микросекундных импульсов излучения. «Радиотехника и электроника», 1979,т.24,№1,c.105-108

17. Scholz M.,Konig R.,Leupold D. Перестраиваемый источник импульсного УФ света. Патент ГДР,кл.21g 53/00, $(\mathrm{H} \quad \mathrm{O} \quad$ IS 3/12),№113828,заявл.07.09.73,опубл.20.06.75

18. Оптический локатор для контроля оптической плотности дымового столбе. "Laser Focus”, 1976, v.I2,№2,p.30-32 
19. Мирошников М.M. Теоретические основы оптико-электронных приборов.Л., « Машиностроение », 1977,с. 77-86

20. Ребрин Ю.К. Управление оптическим лучом в пространстве. М., «Советское радио», 1977,с.225-232

21. Блаут-Блачев Р.Н. Сканирующий пирометр. В сб. «Теория и применение автоматических систем», М., «Наука»,1964,с.288-297

22. Сидоров С.Н. Малогабаритный турбинный двигатель для высокоскоростных фоторегистрирующих устройств. «Успехи науч. фотографии», 1959,т. YI

23. Самуров Л.А. Оптические ускорители и новая схема сверхскоростной кинокамеры. «Успехи науч. фотографии»,1959,т. YI

24. Шнирман Г.Л. Некоторые вопросы развития луп времени и фотохронографов с зеркальной разверткой. «Успехи науч. фотографии», 1953,т. YI,c .93-101.

25. Garbuny M.,Vogl T.P.,Hansen J.R. “ Rev.Sci.Instruction.”, 1957. v. 28,№10 p.286

26. Шарма Б.Л.,Пурохит Р.К. Полупроводниковые гетеро лазеры.М., «Советское радио», 1979

27. Богданкевич О.В.,Дарзнек С.А.,Елисеев П.Г.Полупроводниковые лазеры.М., «Наука»,1976

28. Андреева Л.А.,Егоров М.М., Кайдалов С.А.Степанов Б.М. Генератор световых сигналов для исследования частотных характеристик фотоприемников. «ПТЭ», 1970,№4 ,с. 211-213

29. Вакуленко В.М.,Иванов Л.П.Источники питания лазеров7 М., «Советское радио», 1980

30. Крессел Г.,Ладани А.,Эттенберг М.,Локвуд $Г$. Источники света. «УФН»,1978,т. 126,№4,с.625-639

31. Богатов А.П., Гуров Ю.В., Елисеев П.Г.,Хайретдинов К.А. Аномальная динамика генерация в полупроводниковых лазерах с несимметричной волноводной волноводной структурой. «Кватовая электроника», 1978,т.5,№11,с.2402

32. Данилов В.А.,Зенченко С.А., генератор оптических импульсов на АИГ:Nd лазере. «Кватовая элктроника»,1978,т.5,№11,с.2447

33. Алферов Ж.И., Дианов Е.М.,Прохоров А.М. и др. Волоконно-оптическая линия передачи сигналов для систем дальней связи на длине волны 1,3мкм. «Квантовая электроника»,1978,т.5,№11,с. 2486

34. Страховский Г.М.,Успенский А.В. Основы квантовой электроники.М., «Высшая школа»,1979

35. Левин Б.Р Теоретические основы статической радиотехники. Кн.1,М.,1974.

36. Хапкевич А.А Основы радиотехники.М.,1962

37. Макэвой,Крист.Спетры модулированных последовательностей импульсов». «ТИИЭР»,т.53,№9,c.1411-1412,(1965).

38. ЛИ. Спектры мощности когерентных и некогерентных последовательностей импульсов. «ТИИЭР»,1966,т.54,№9,с.63 Росс М.Лазерные приемники. М., «Мир»,1969 
39. Магдич Л.Н., Молчанов В.Л. Акустооптические устройства и их применение. М., «Советское радио», 1978.

40. Петров М.П. и др. Акустооптический дефлектор лазерного изучения. В сб. «Оптическая обработка информации». Под ред. С.Б. Акустооптические устройства спектрального и корреляционного анализа сигналов. Л., «Наука», 1978. с.21-25.

a. Кулаков С.Б. Акустооптические устройства спектрального и корреляционного анализа сигналов. Л., «Наука», 1978.

41. Сб. статей: Квантовая электроника. Труды ЛПИ, №344, Л., 1975.

42. Пуговкин А.В. Преобразование изображений в брэгговских акустооптических модуляторах. «Письма в ЖТФ», 1980, 6, №13,с.790-793.

43. Корпел А. Акустооптика обзор основных принципов. «ТИИЭР»; Пер.с. англ., 1981г. Т.69, №1, с.55-62.

44. Родес У.Т Акустооптическая обработка сигналов: Свертка и корреляция. «ТИИЭР»; Пер.с. англ., 1981г. Т.69, №1, с.74-91.

45. Терпин Т.М. Спектральный анализ сигналов оптическими методами. «ТИИЭР»; Пер.с. англ., 1981г. Т.69, №1, с.92-108.

46. Янг Э.Х., Яо Шикай. Расчет акустооптических устройств. «ТИИЭР»; Пер.с. англ., 1981г. Т.69, №1, c.62-74.

47. Борсак Дж.М. Фотодетекторы для акустооптических систем обработки сигналов. «ТИИЭР»; Пер.с. англ., 1981г. Т.69, №1, с.117-137.

48. Кучеров И.Я. Управление параметрами лазерного излучения в диффузном волноводе при помощи нормальных упругих волн в подложке. "Письма в ЖТФ”,1980, 6, №24, c.1516-1519.

49. Lee Jim P.Y. Acoustooptic spectrum analysis of radar signals using an integrating potodetector array. “Appl.Opt.”, 1981, Vol.20, №4, p.595-600

50. Alippi A. a.al. Real time acoustooptical spectrum analyzer through unguided light - surface acoustic waves interaction. “Opt. Commun.”, 1980, Vol.35, №1, p.37-41

51. Barbe D.F. a.al. Signal processing with charge - coupled devices. "Charge Coupled Devices", Berlin e.a., 1980, p.91-145

52. Vander Laugt A. Interferometric spectrum analyzer. "Appl.Optics", 1981,Vol.20, №16, p.2770-79

53. Зверев В.А., Орлов Е.Ф. Оптические анализаторы. М., “Советское радио”, 1971.

54. Зверев В.А. и др. Авт. свид. (СССР) №410327, опубл.05.01.74 бюлл. , 39, № 480092, опубл. 25.08.75, бюлл.№ 29; № 523360, опубл. 30.07.76, бюлл.№ 28.

55. Гущин В.В и др. Оптическая обработка сигналов с повышенным разрешением в некогерентном свете. Тезисы докладов Ш Всес.школы по оптич.обработке информации, ч.I. Рига, 1980, с.91-93.

56. Зверев В.А. и др. Оптические методы обработки информации с использованием некогерентного света. Сб. "Оптические методы обработки информации”, Л. , “Наука”, 1974. 
57. Гинзбург В.М. , Степанов Б.М. Голографические измерения. М. , “Радио и связь”, 1981.

58. Васильев Ю.Г. и др. Акустооптическое устройство. Тезисы докладов Ш Всес.школы по оптич.обработке информации, ч.І.Рига, 1980, с.163.

59. Егоров Ю.В., Наумов К.П. Акустооптический демодулятор частотномодулированных сигналов.Межвузовский сб.научн.трудов "Вопросы анализа и синтез радиосигналов", вып.І,Рязань, 1976.

60. Berg N.I. a.al. Surface wave delay line acoustooptic devices for for signal processing. “Appl.Optics”, 1979, p. 2767-2774.

61. Корбуков Г.Е. , Куликов В.В. и Цветов Е.Р. Оптический гетеродинный метод корреляционный обработки изображений. В сб.: "Голография и обработка информации" , Л. , Наука , 1976, с.51-69.

62. Егоров Ю.В., Ушаков В.н. Опорный транспортант для акустооптических корреляторов. Авт.свид.(СССР) № 605185. Бюлл.изобр. № 16, 1978.

63. Адрианова П.И. , Заславская В.Р. Детектирование фазовомодулированного света гетеродинными методом. “ОМП”, 1972, № 10, с.ІІ.

64. Визен Ф.Л. и др. Исследование отклонения луча инжекционного лазера акустическим дефлектором .Тезисы докладов Ш Всес.школы по оптич.обработке информации , ч.П, Рига,1980, с.225.

65. Dixon R.W. Optical investigation of magnetically induced elastic wave dispersion in YIG. "I. Appl. Phys." 1967, Vol.38, p.3634-40

66. Dixon R.W., Matthews H. Diffraction of light by elastic waves in YIG. "Appl. Phys. Letters", 1967, Vol.10, p.195-7

67. Auld B.A., Wilson D.A. Spin and acoustic Bragg diffraction in longitudinal magnetoelastic waves . "Appl. Phys. Letters", 1967, Vol.11, p. 368-70.

68. Smith A.W. Diffraction of light by Magnetoelastic waves. "Appl. Phys. Lett", 1967, Vol. 11, p. 7-9.

69. "IEEE Trans. On Sonics and Ultrasonics", 1968, Vol.su-15, 3, p. 1961-167.

70. Штраус В. Магнитоупругие свойства феррита-граната. В кн. "Физическая акустика" под ред. У.Мезона, т.ІУ, ч. Б. Применение физической акустики в квантовой физике и физике твердого тело. М. , "Мир", 1970. 


\section{Иллюстрации}

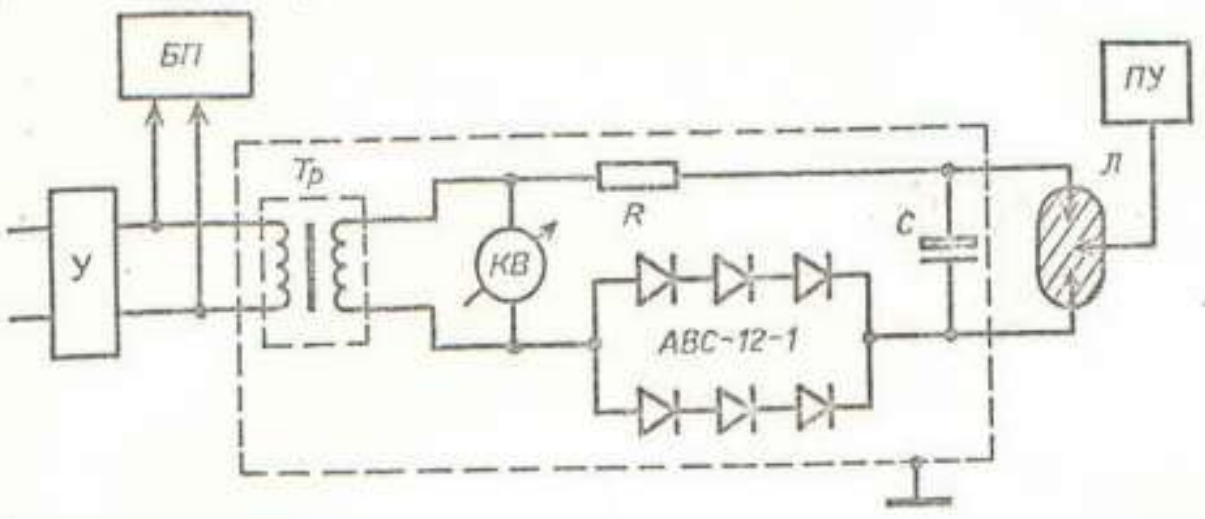

Pnc. 1.1

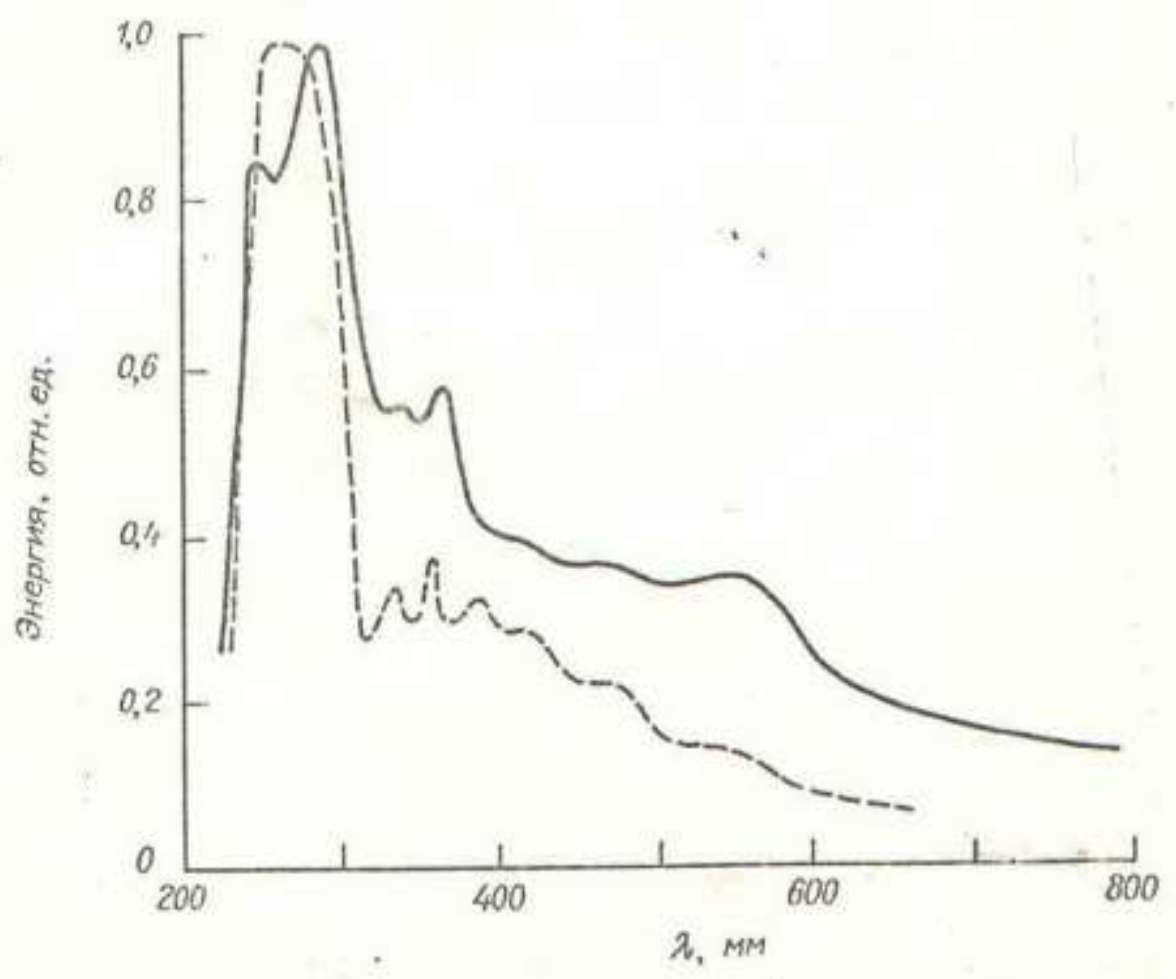

Puc. 1.2 


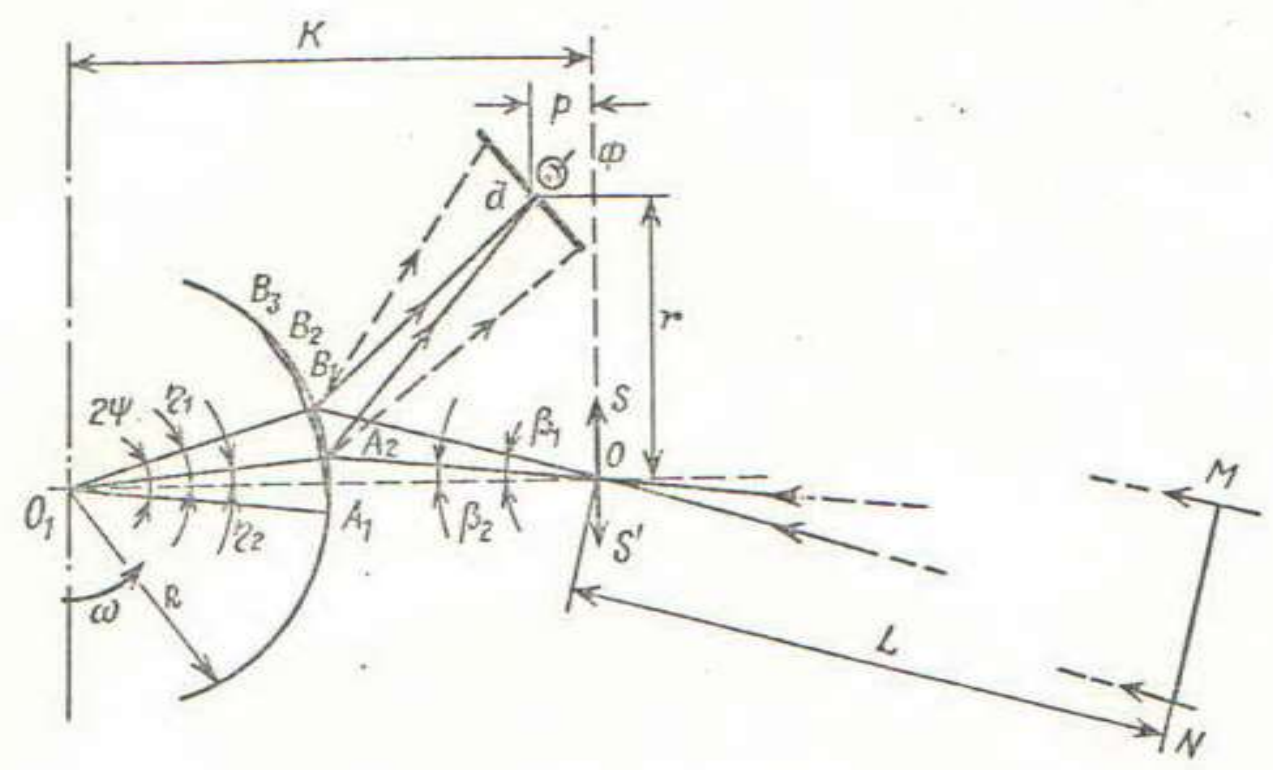

Pnc. 1.3

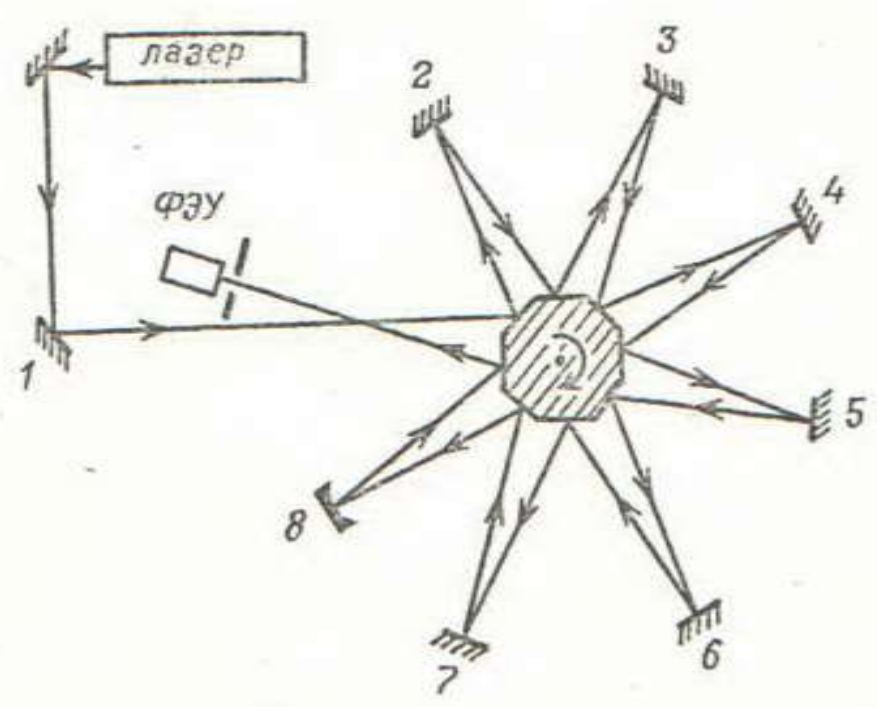

Pnc. 1.4

75 


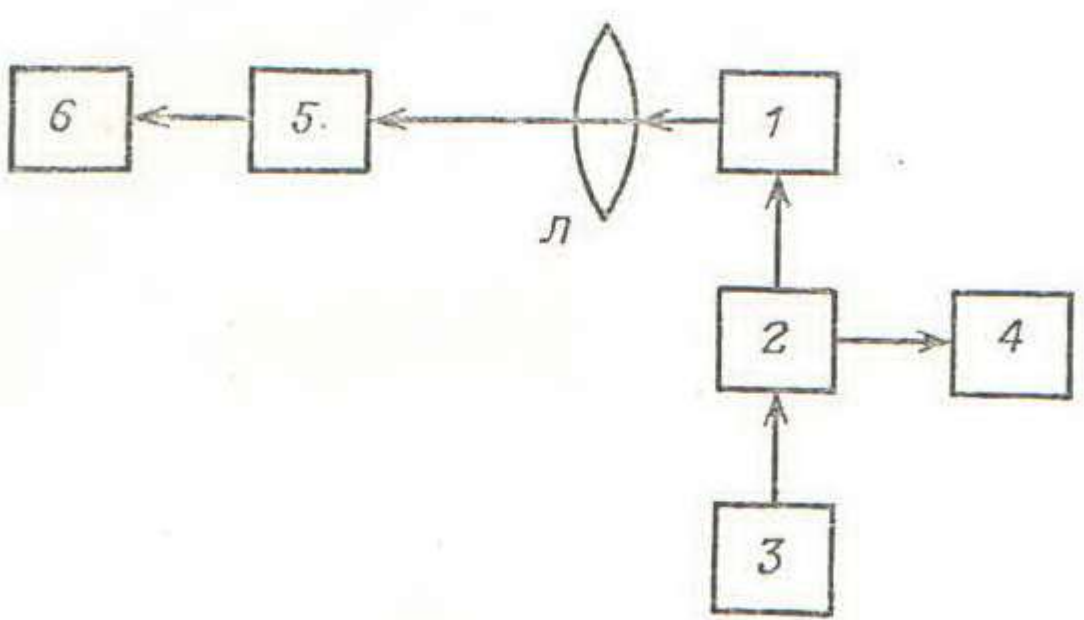

Puc. 1.5

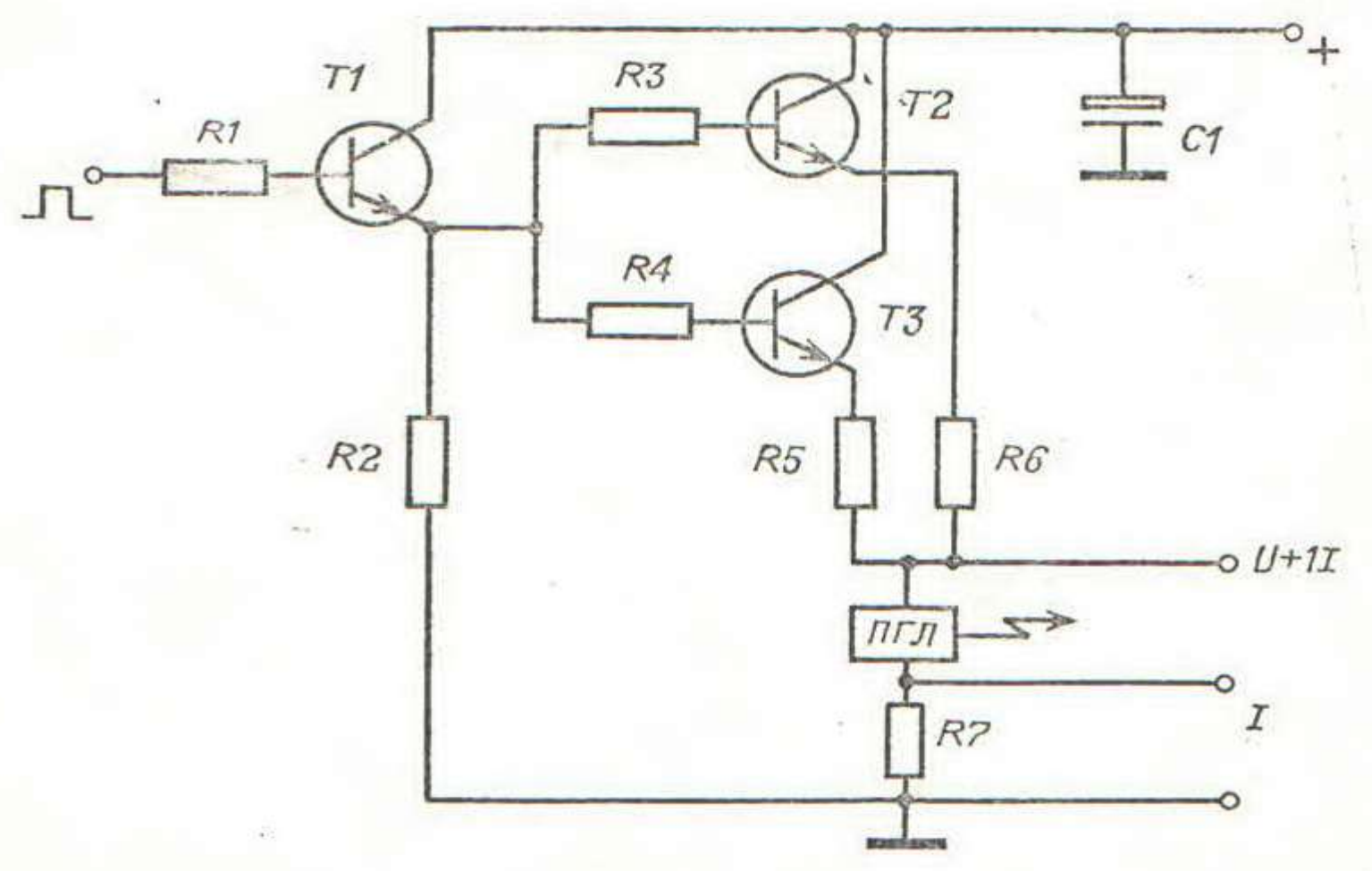

Puc. 1.6

76 


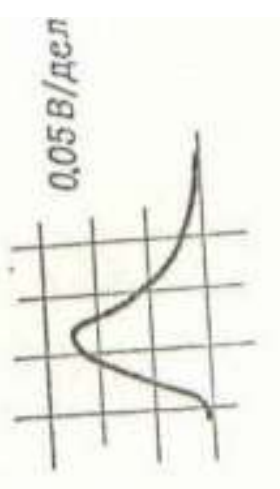

๕

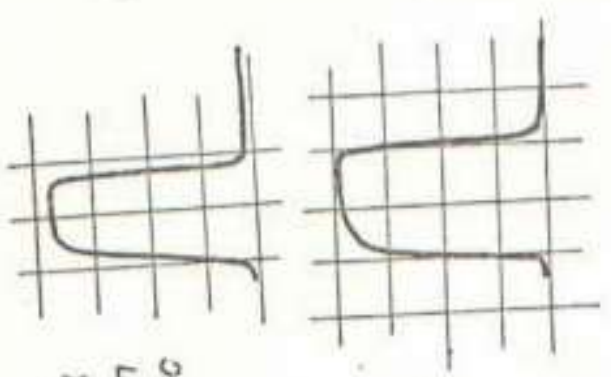

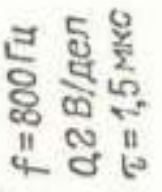

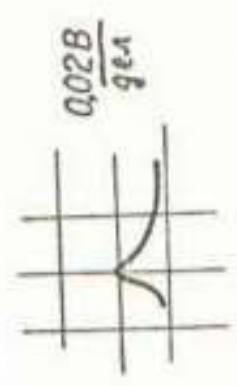

ลิ

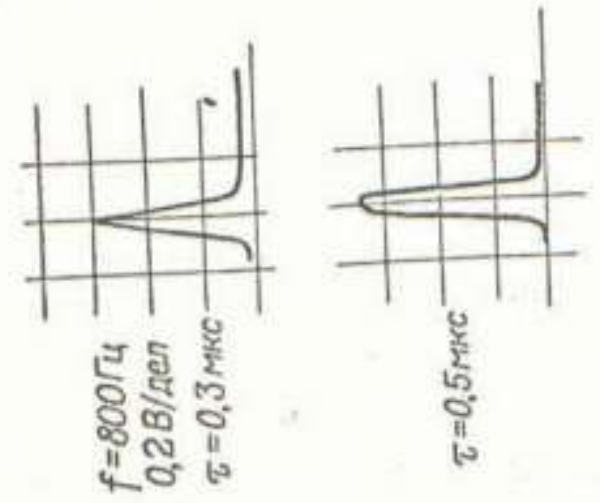

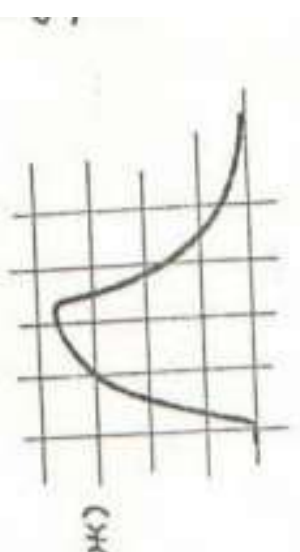

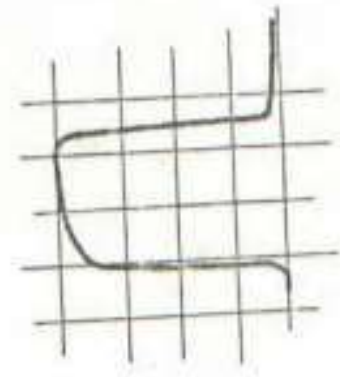

in

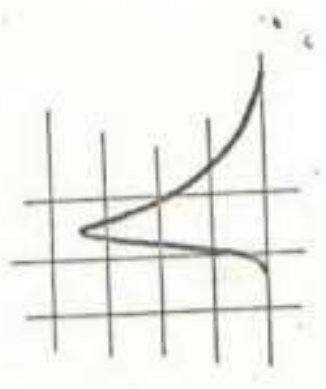

ิิ
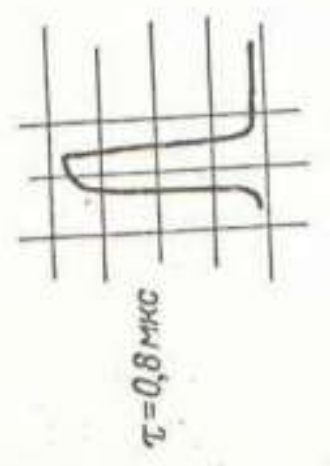

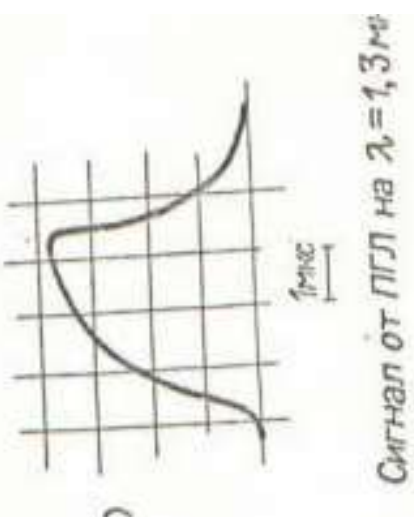

के

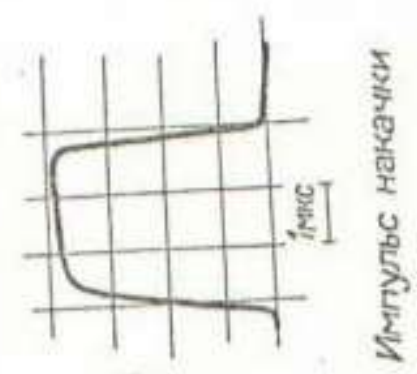

$y$
$\frac{1}{2}$
0
0
11
10

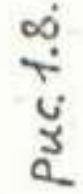

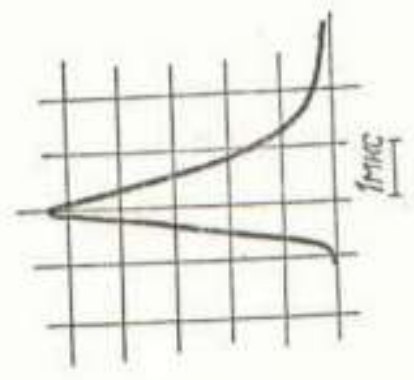

ᄃ

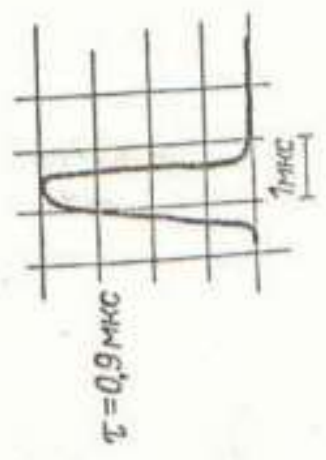




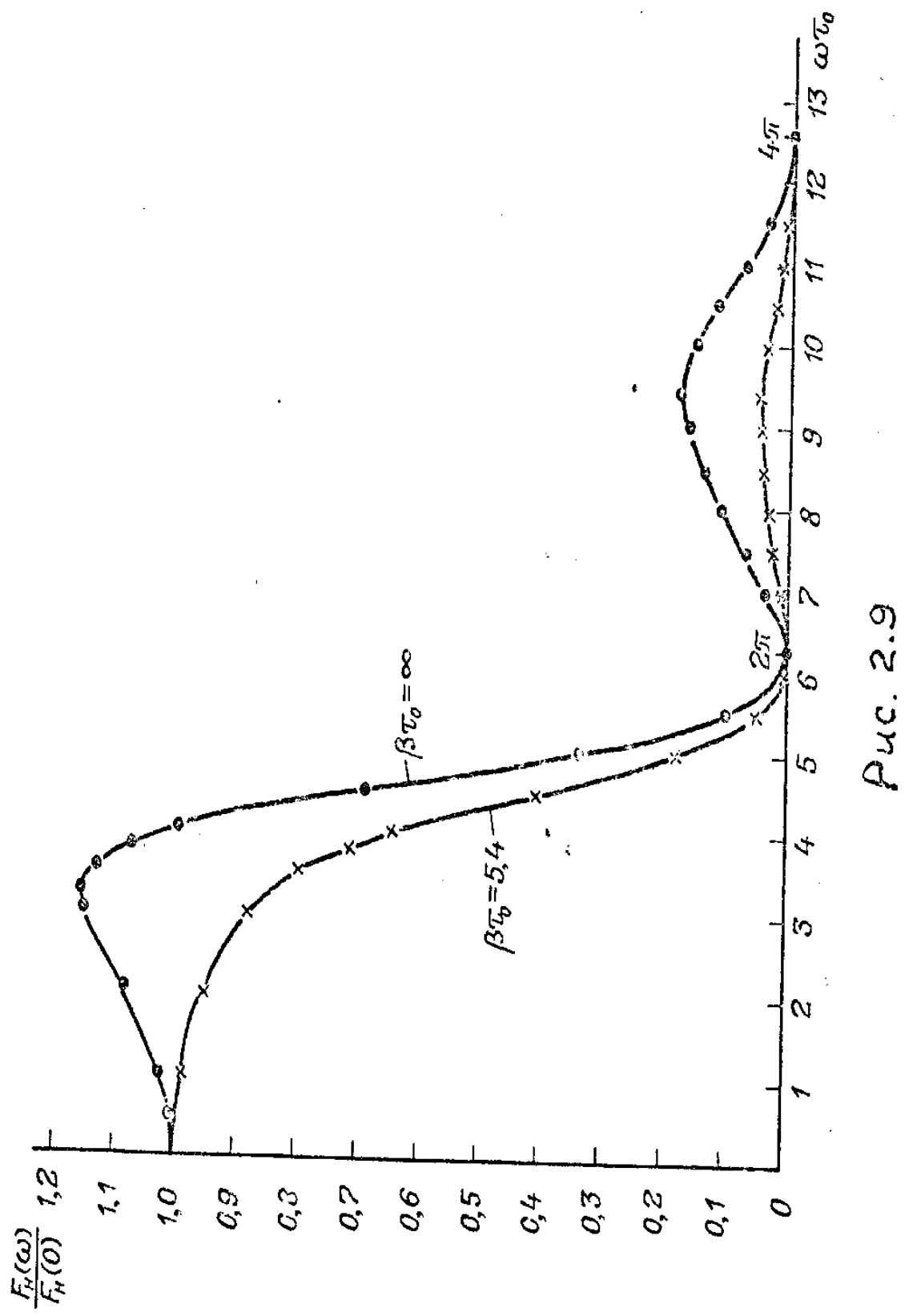




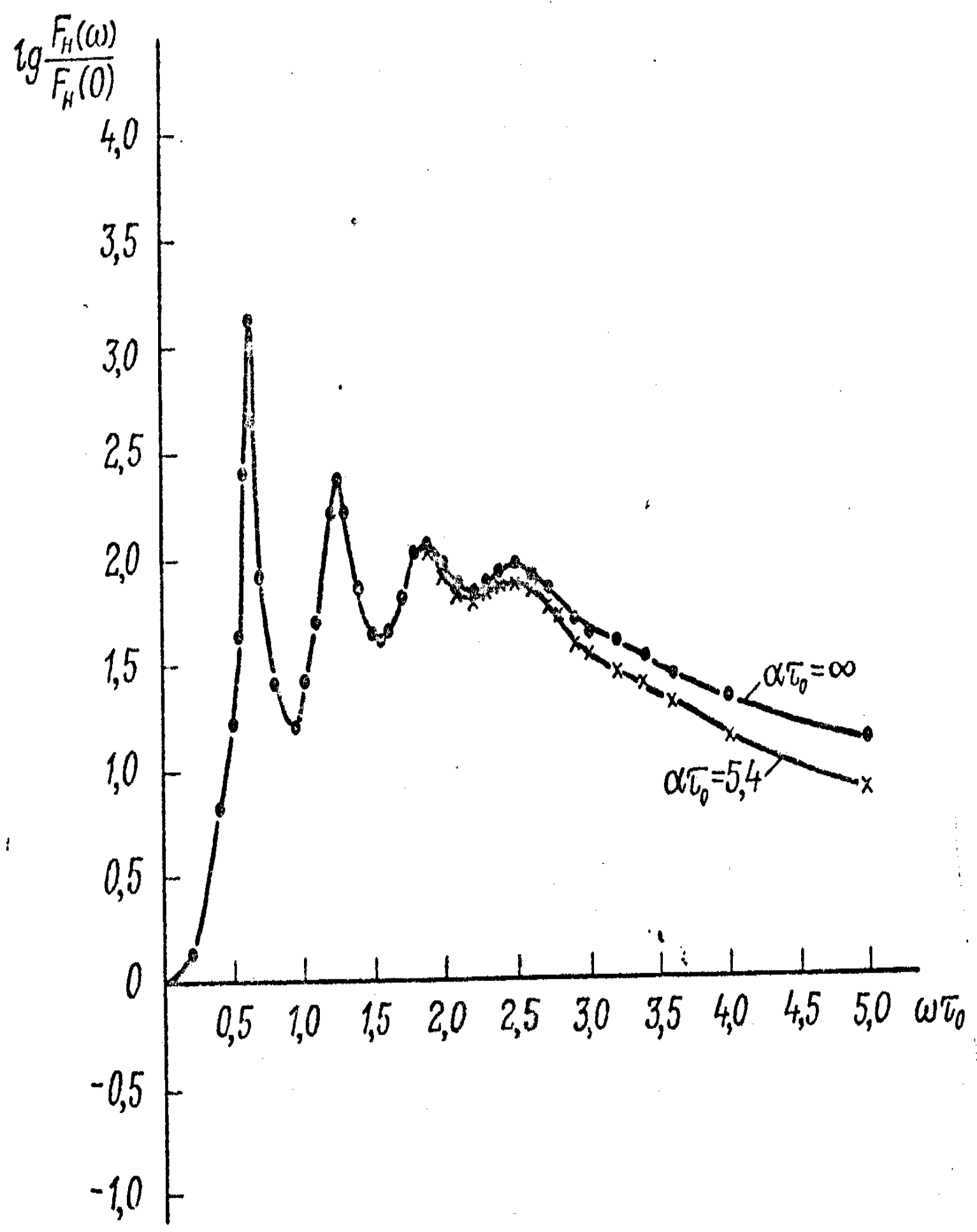

Рис. 2.10 

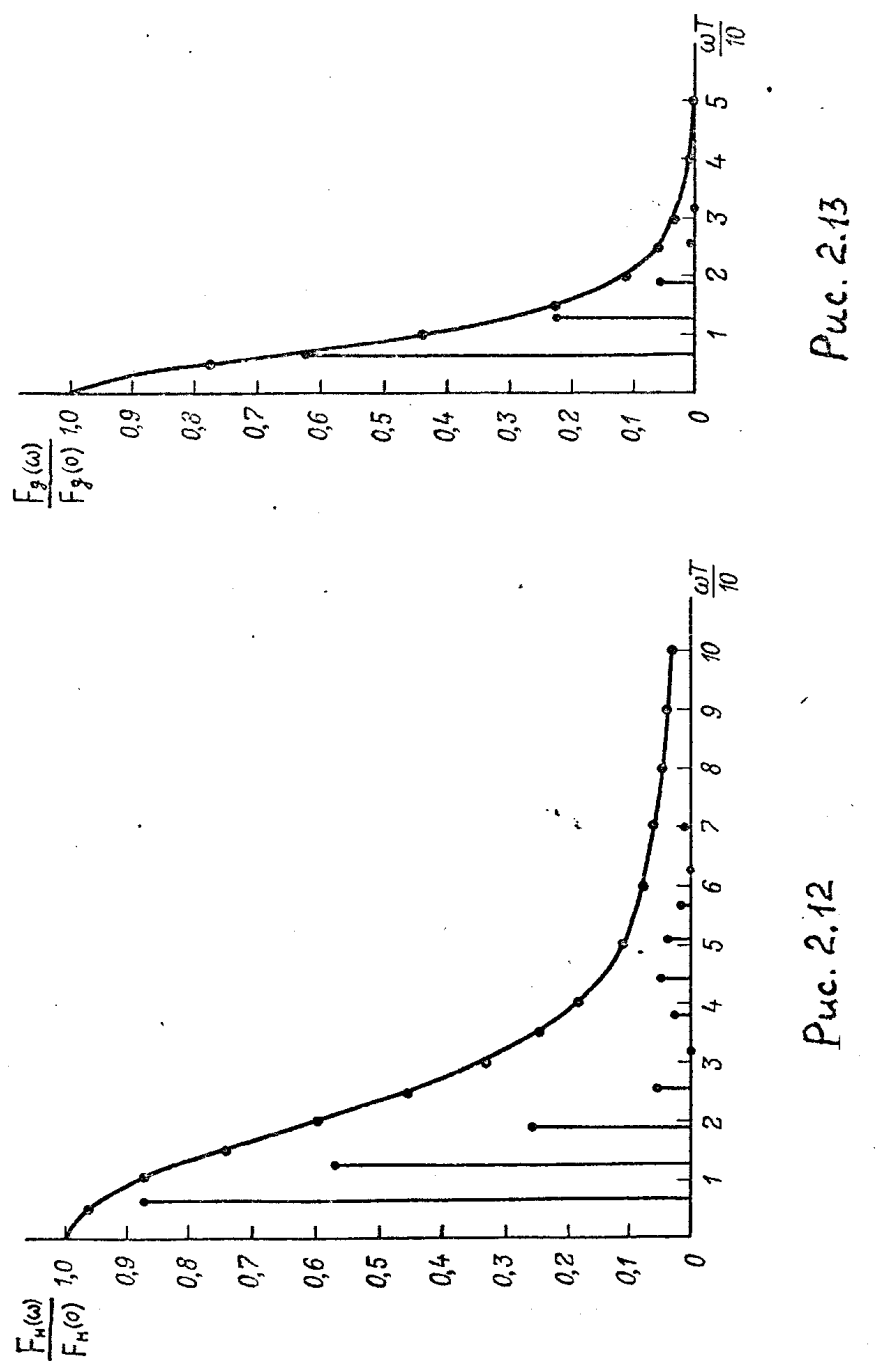


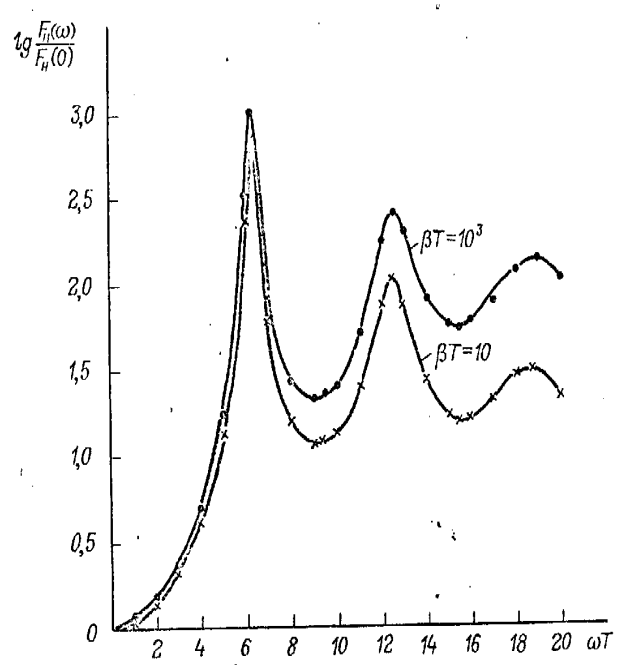

Рис. 2.14 


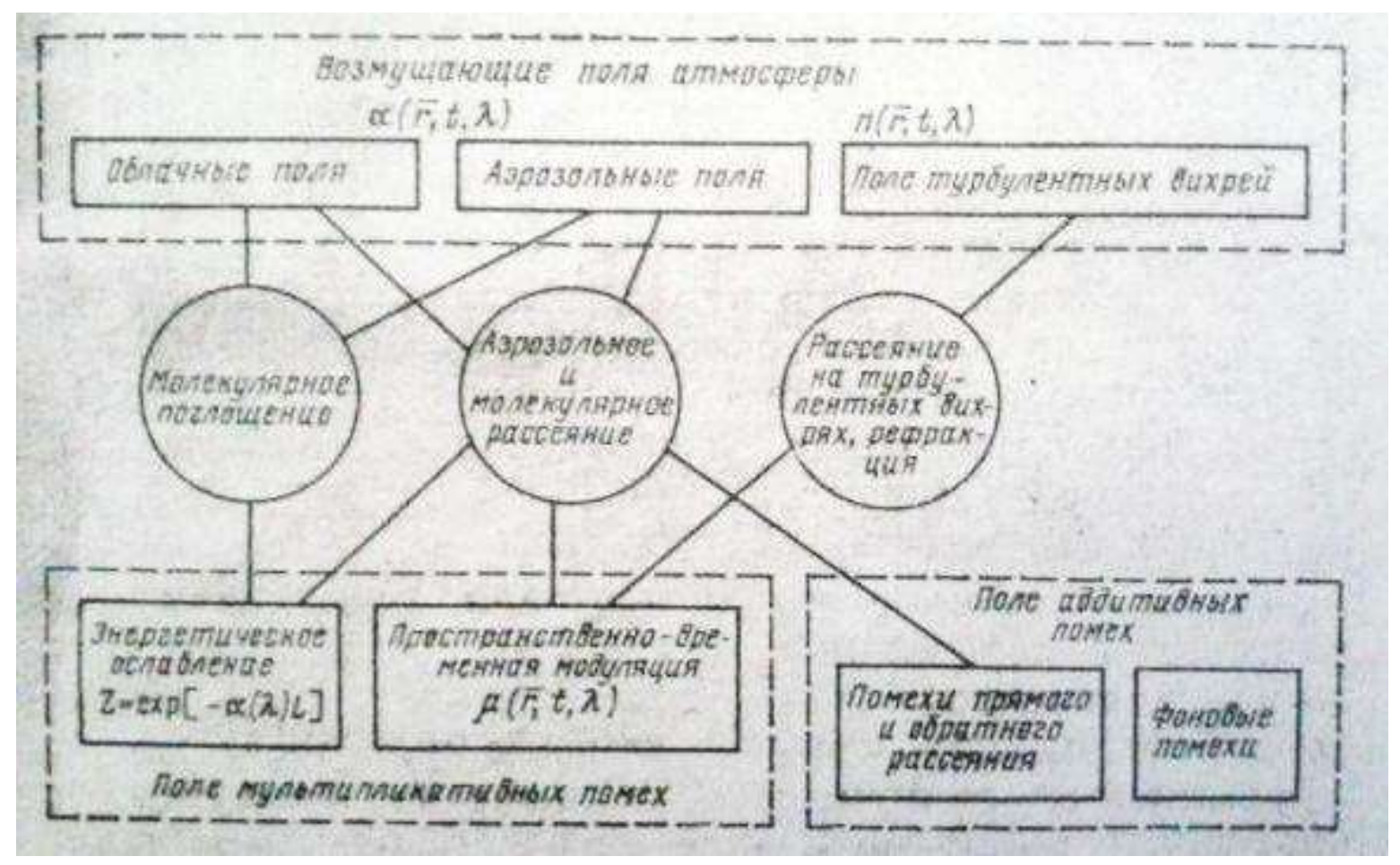

РИС. 3.1. Классификация возмущающих полей атмосферы, эффекты их взаимодействия с когерентными оптическими полями и характер возникающих при этом помех [1]

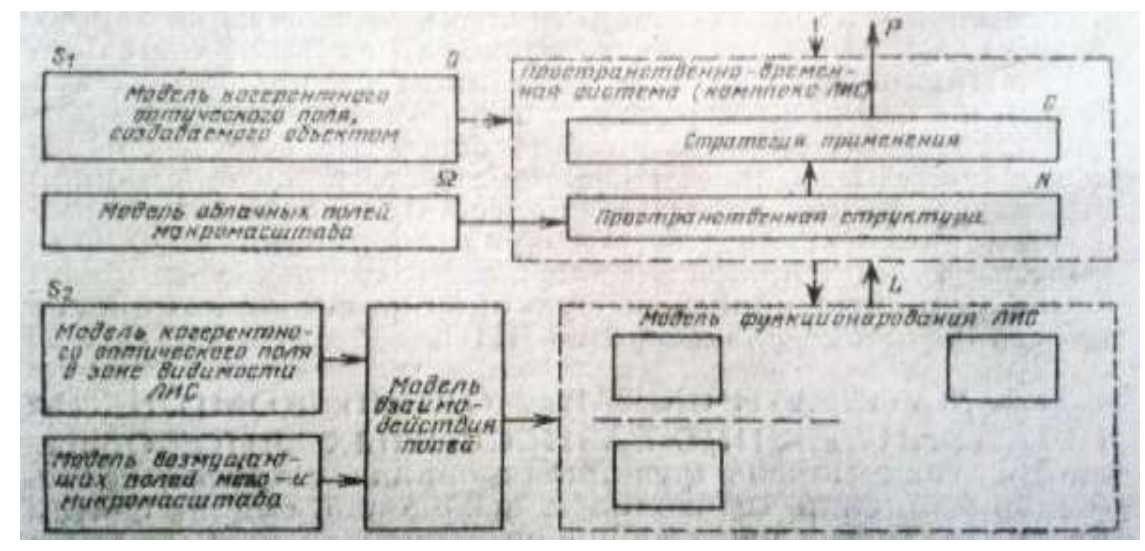

РИС. 3.2. Общая модель комплекса ЛИС 

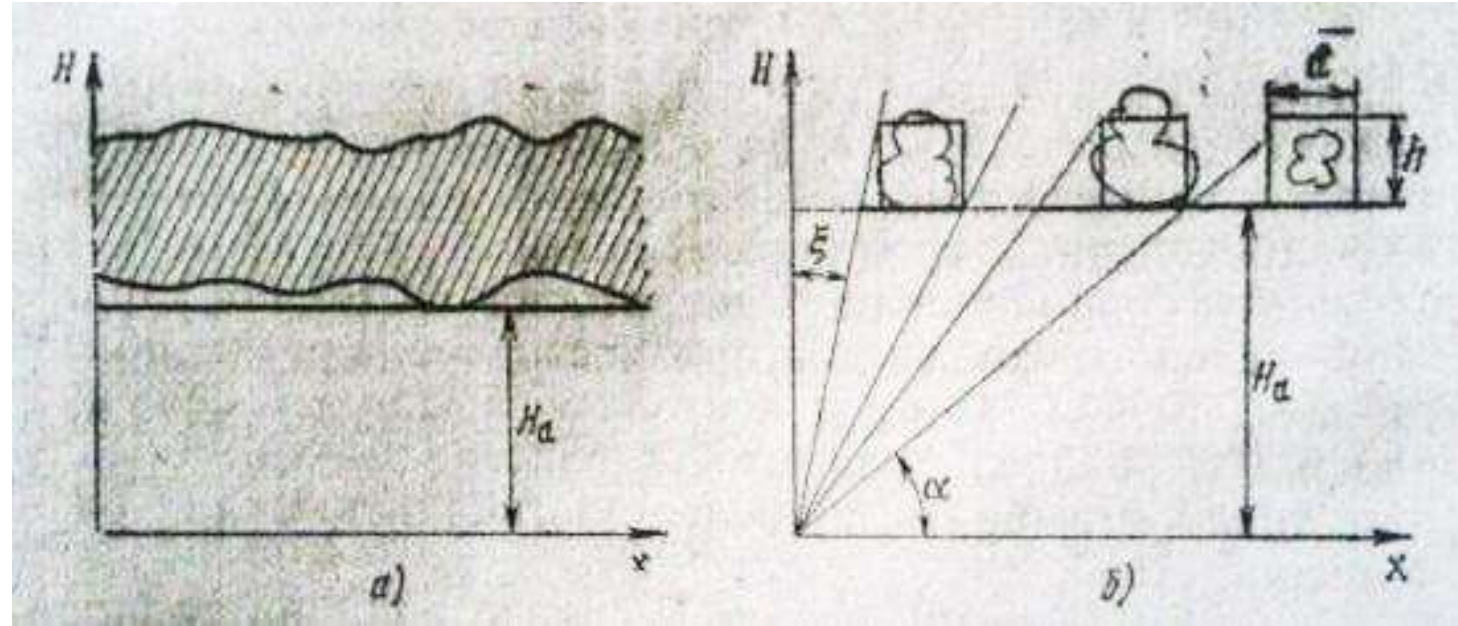

РИС. 3.3. Модели полей облачных неоднородностей: $\boldsymbol{a}$ - поле сплошной облачности (заштрихованная часть), в общем случае может быть многоярусным; $\boldsymbol{\sigma}$ - поле с дискретными неоднородностями

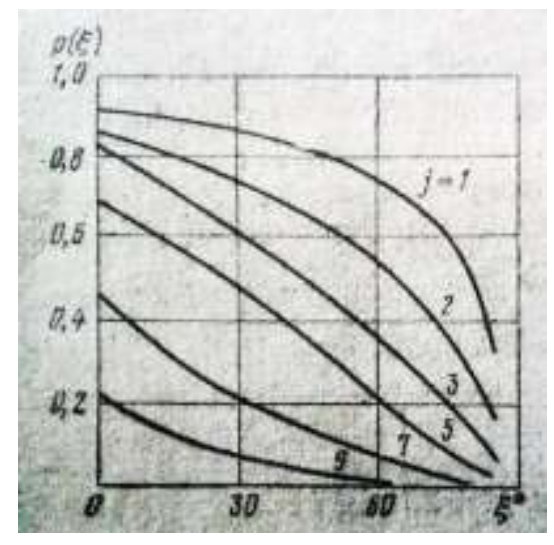

РИС. 3.4. Вероятность свободной линии визирования в заданном направлении $\xi$ для поля кучевой облачности Ґ23] 


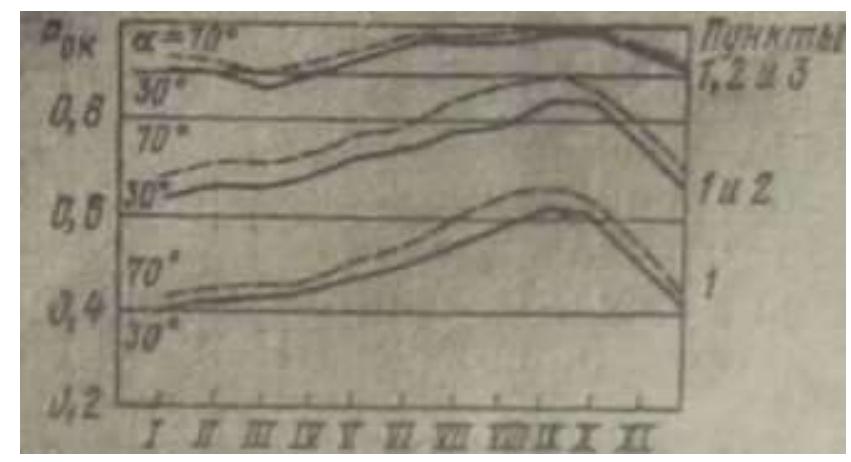

РИС. 3.5. Вероятность оптического контакта с ИСЗ хотя бы одним из нескольких пунктов: 1-Вашингтон (окр. Колумбия), 2-Омаха (шт. Невада), 3 -юго-западный район США [30]

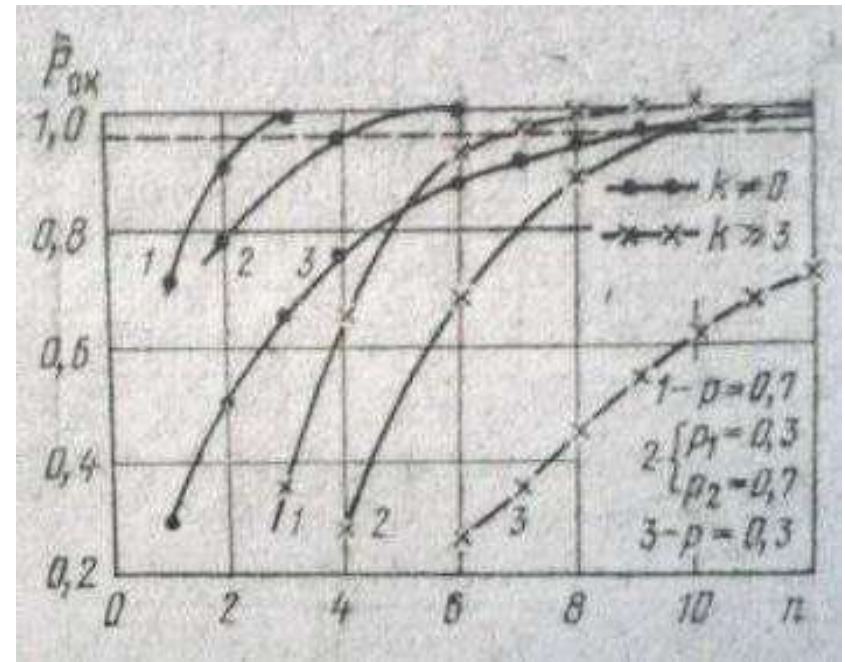

РИС. 3.6. Зависимость вероятности оптического контакта для различных стратегий применения $(k \neq 0$ и $k \geq 3)$ от числа и условий размещения пунктов 


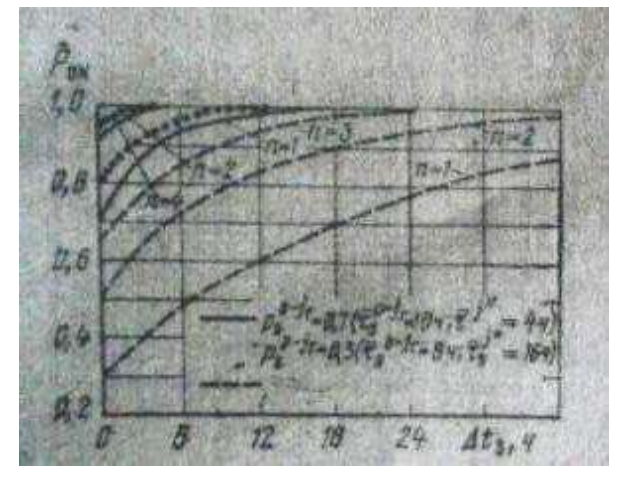

РИС. 3.7. Зависимость вероятности оптического контакта $P_{\text {Ок от }}$ величины интервала оперативности $\Delta t_{3}$

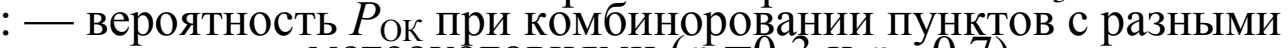
метеоусловиями $\left(p_{\mathrm{s}}=0,3\right.$ и $\left.p_{\mathrm{s}}=0,7\right)$

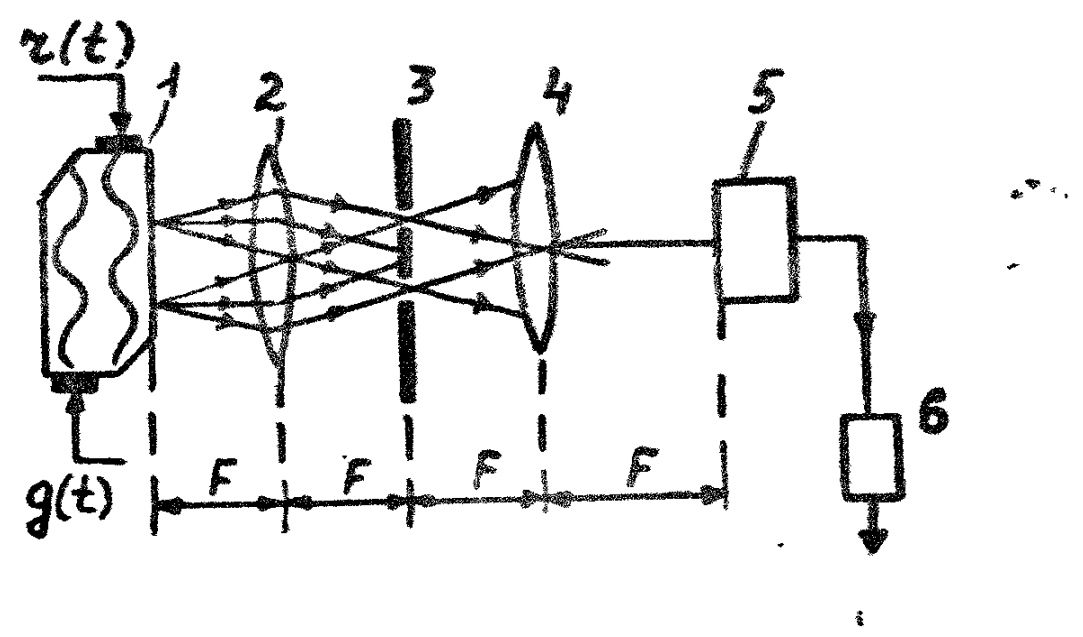

Рис.4.1. Схема ОА процессора в режиме Рамана - Ната.

1- Два совмещенных ультразвуковых модулятора света, 2 Фурье - преобразующая линза , 3 - пространстранственный фильтр (диафрагма), 4- интегрирующая линза, 5 - фото-приемник, $\epsilon$ электрический фильтр . Ғ - фокусное расстояние линза. 

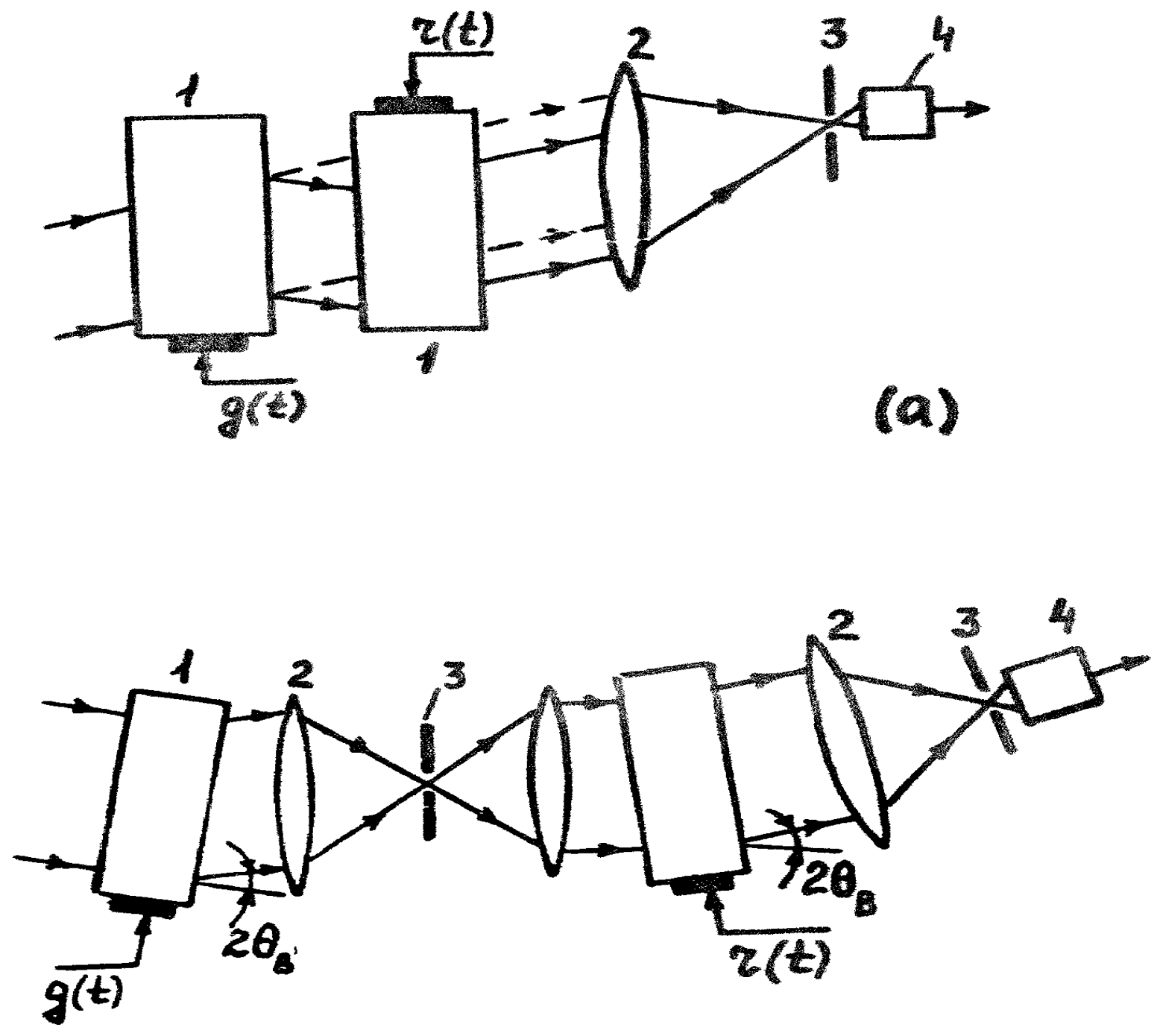

( $\delta)$

Рис.4.2. Схемы OА процессоров в режиме Брэгга: (a) -c когерентным детектированием, (б)-с некогерентным детектированием. 1- ОА ячейка, 2линза, 3- диафрагма, 4- фотоприемник. 


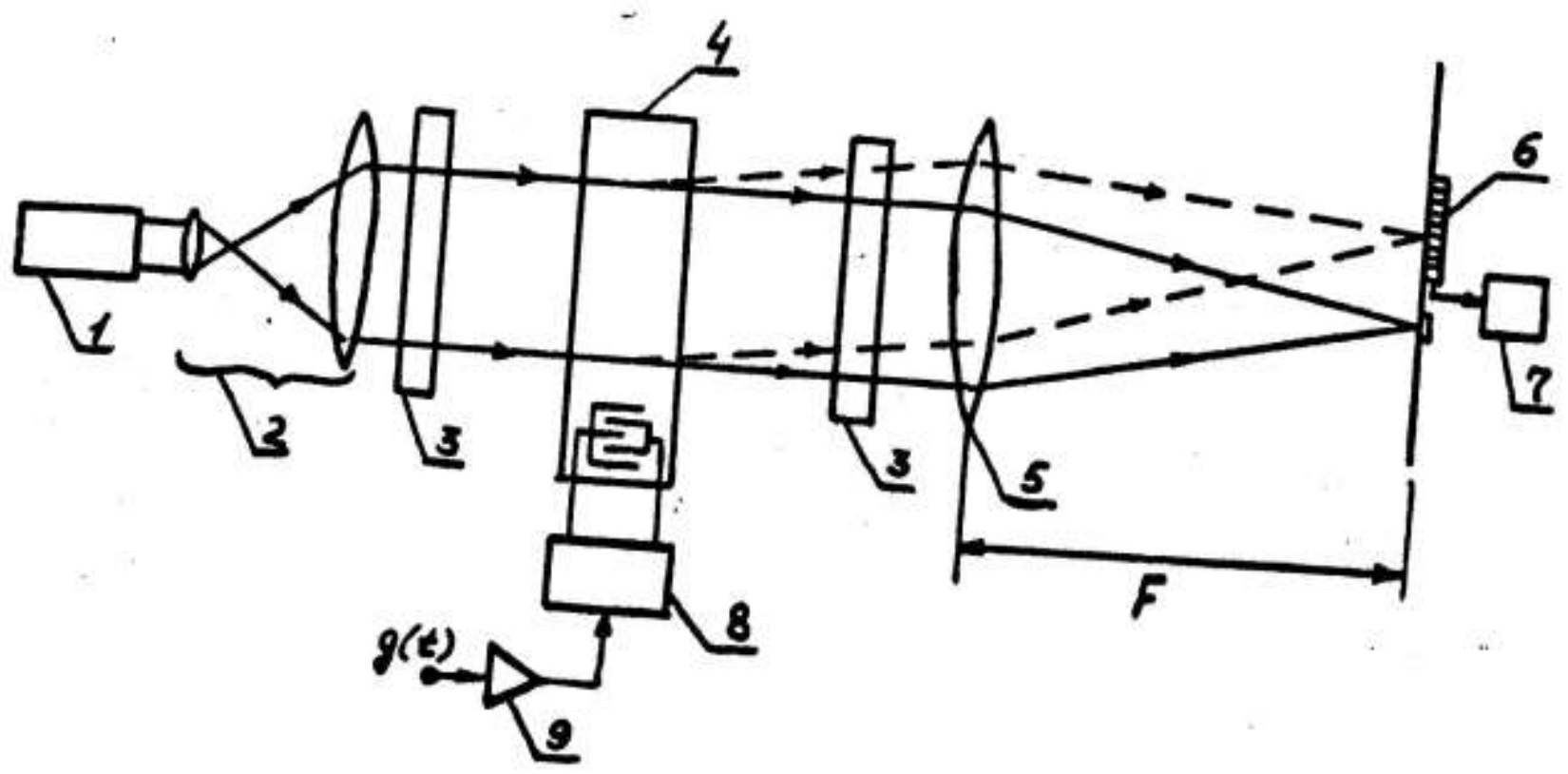

Рис.4.3. Схема ОА спектроанализатора на ПАВ : 1-лазер, 2-коллиматор, 3цилиндрические линзы, 4-OA ячейка из ниобата лития с пьезопреобразователями в виде встречно-штыревых электродов для возбуждения ПАВ, 5-Фурье -преобразующая линза, 6- ПЗС линейка, 7устройство обработки серии видеосигналов, 8-широкополосная согласующая схема, 9-широкополосный усилитель. 


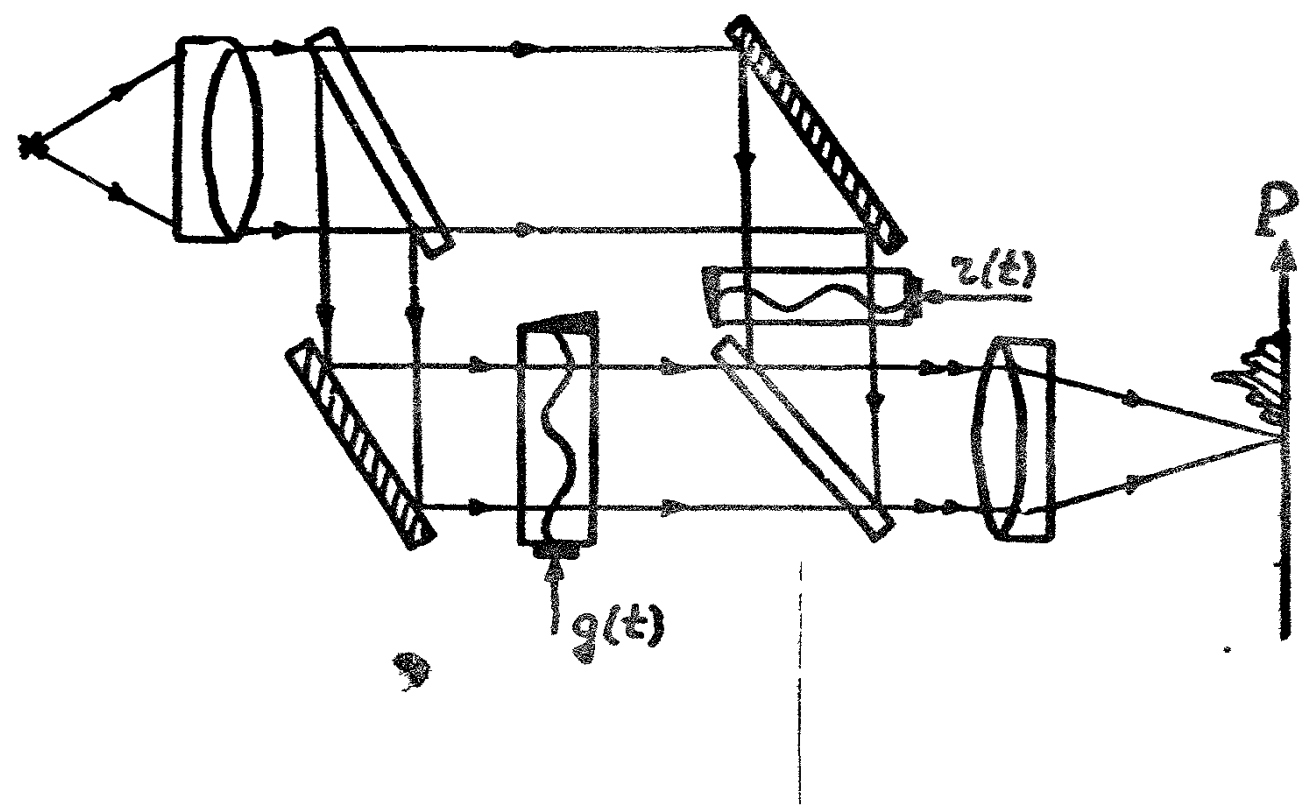

Bud \& $\phi_{\text {ypse-nnockoctu } P}$

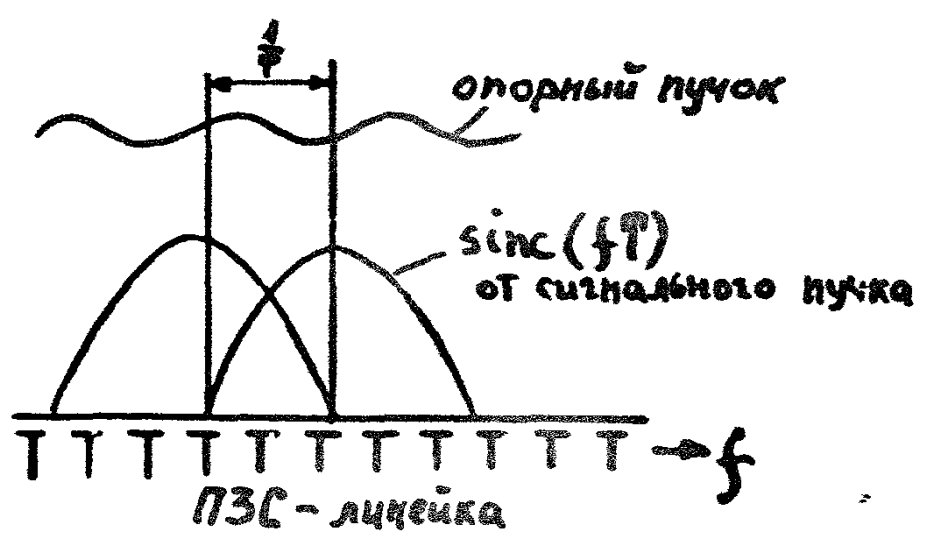

Рис.4.4. Интерферометрический оптико-акустический спектроанализатор [15]. 


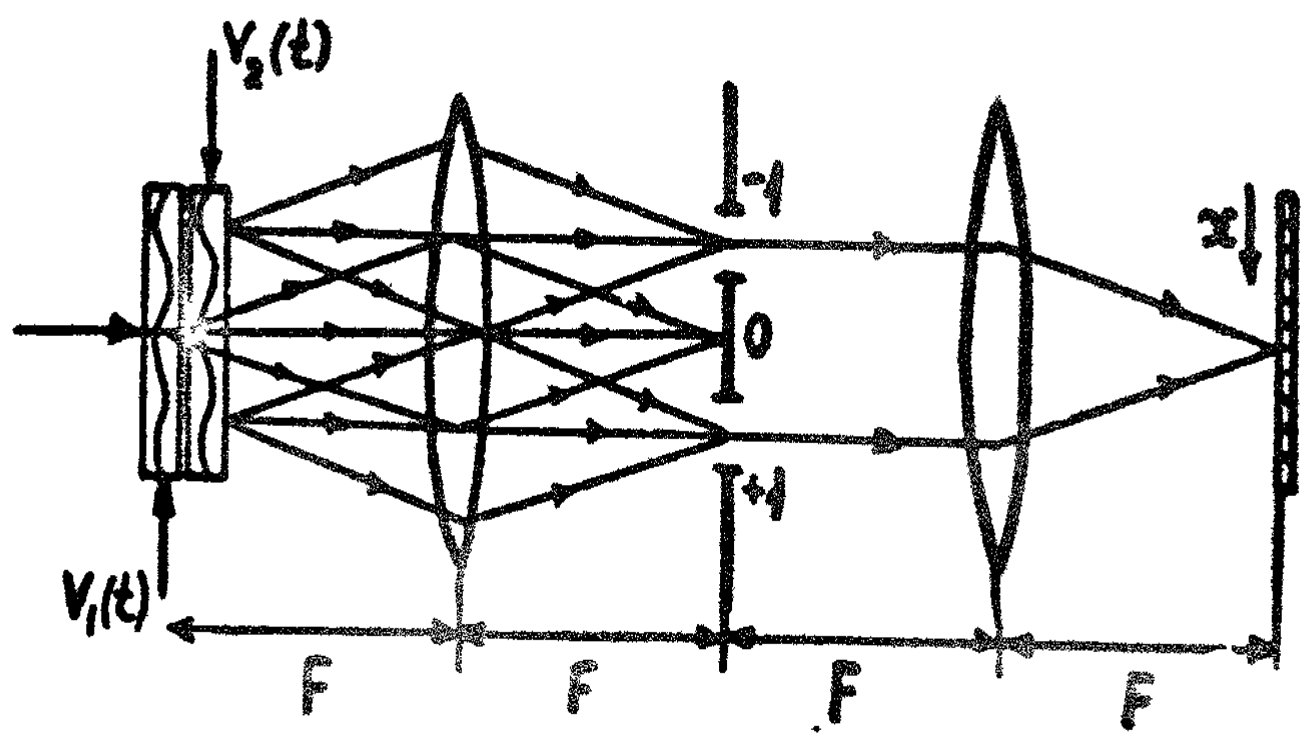

Рис.4.5 Коррелятор с двумя ОА ячейками. в котором осущестелеяется интегрирование сигнал по времени с помощью ПЗС -- линейки [ 7 ]

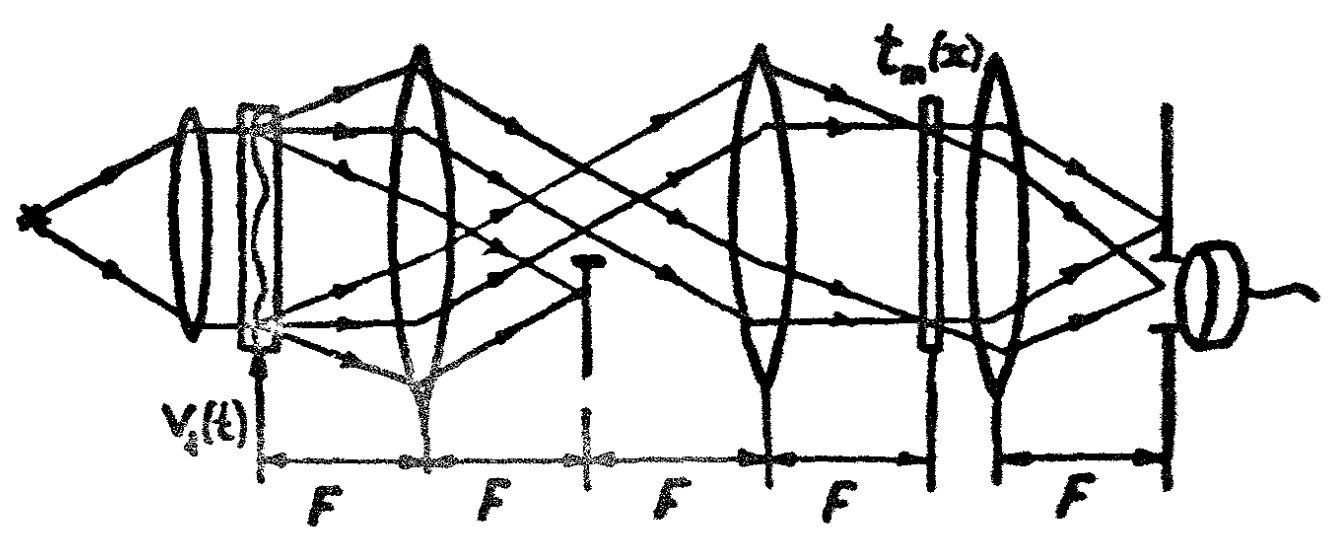

Рис.5.1. Оптика - акустический гетеродинный коррелятор [ 7 ] с фотосмешением нулевого и первого дифракционных максимумов 

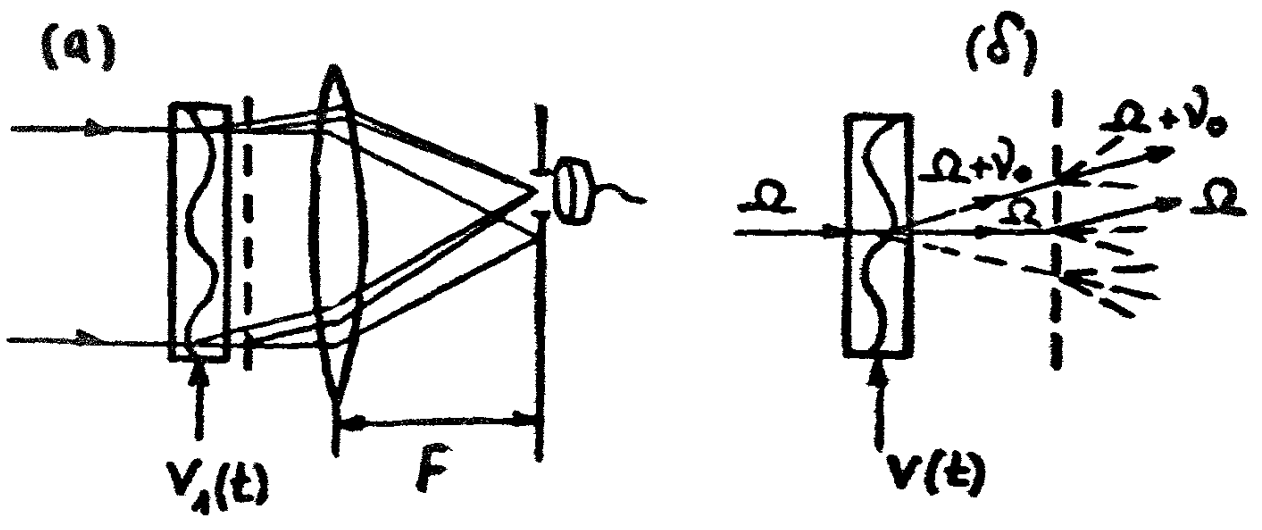

Рис.5.2. Оптика - акустический гетеродинный коррелятор [ 7 ] : (а) - базовая система , ( б ) - схема, поясняющая возникновение компонент, приходящих на фотодетектор. 


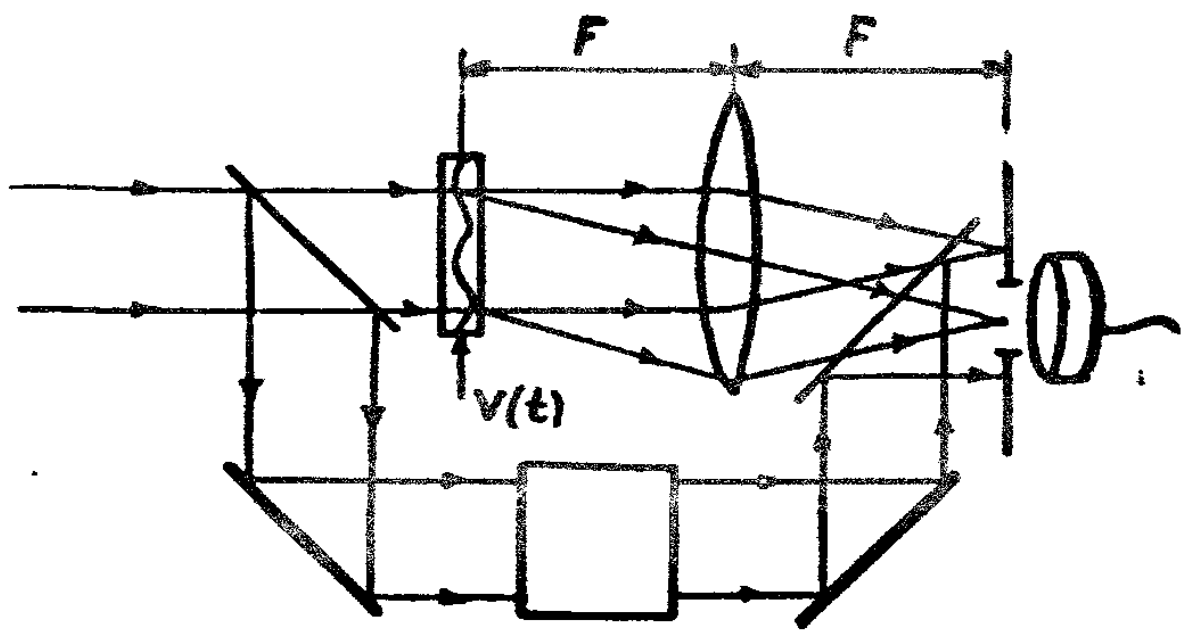

Рис.5.3. Оптико-акустический гетеродинный коррелятор [1] с фото смешением опорной и сигнал волн в плоскости преобразования фурье. 


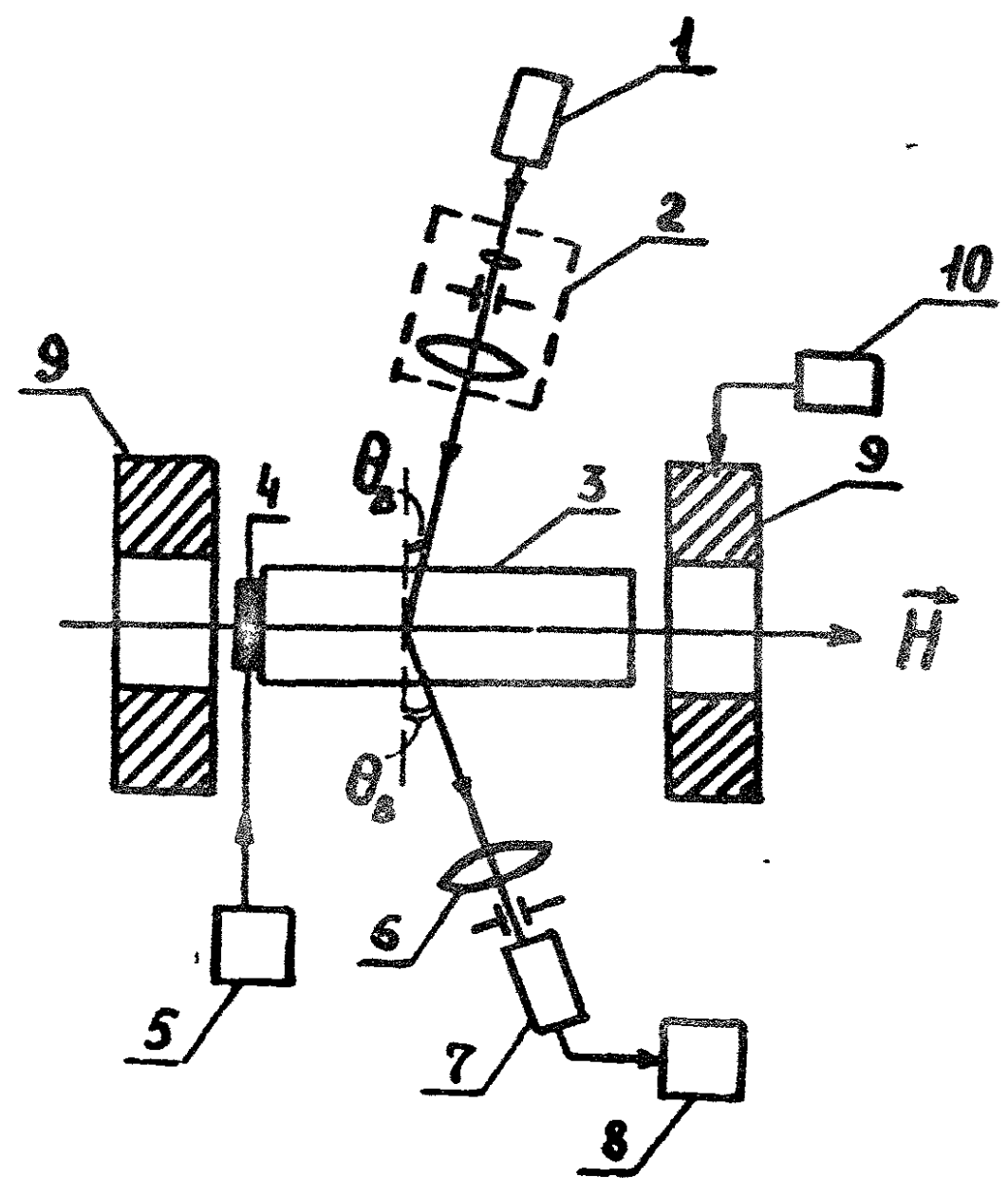

Рис.5.4 Магнитоакустооптический дефлектор[31] 


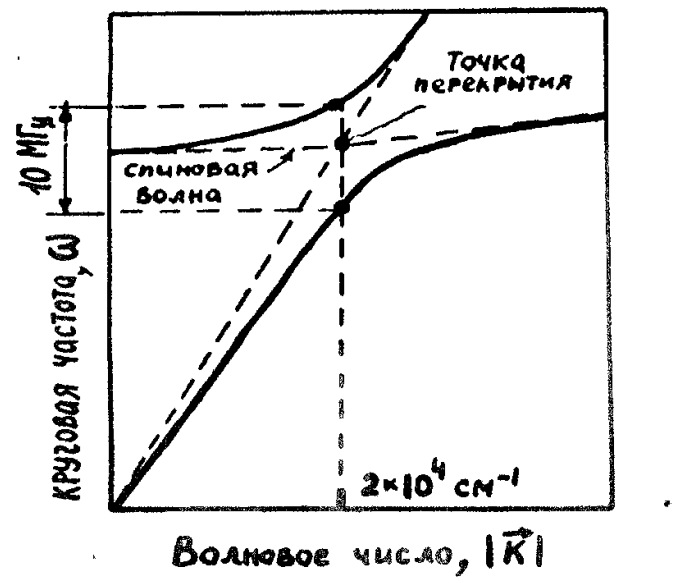

Рис.5.5. Дисперсионные кривые поперечых магнитоупругих воли, распростанящихся вдоль магнитного поля в КИГ- кристалле

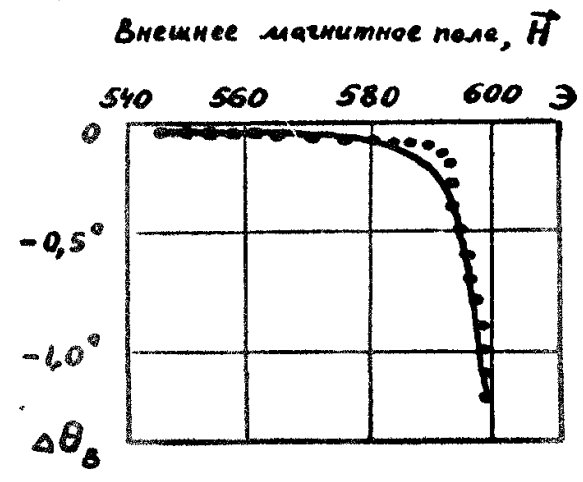

Рис.5.6. Изменение брэгговского угла дифракция в зависимости от величины магнитного поля 